Demonstrações assistidas por computador para equações diferenciais ordinárias

Mário Cesar Monteiro do Prado 



\title{
Demonstrações assistidas por computador em equações diferenciais ordinárias
}

\author{
Mário Cesar Monteiro do Prado
}

Orientador: Prof. Dr. Marcio Fuzeto Gameiro

Dissertação apresentada ao Instituto de Ciências Matemáticas e de Computação - ICMC-USP, como parte dos requisitos para obtenção do título de Mestre em Ciências - Matemática. VERSÃO REVISADA

USP - São Carlos

Abril de 2015 
Ficha catalográfica elaborada pela Biblioteca Prof. Achille Bassi e Seção Técnica de Informática, ICMC/USP, com os dados fornecidos pelo(a) autor(a)

\begin{tabular}{|c|c|}
\hline \multirow[t]{3}{*}{ P896d } & $\begin{array}{l}\text { Prado, Mário Cesar Monteiro do } \\
\text { Demonstrações assistidas por computador para } \\
\text { equações diferenciais ordinárias / Mário Cesar } \\
\text { Monteiro do Prado; orientador Marcio Fuzeto } \\
\text { Gameiro. -- São Carlos, } 2015 \text {. } \\
\quad 81 \mathrm{p} \text {. }\end{array}$ \\
\hline & $\begin{array}{l}\text { Dissertação (Mestrado - Programa de Pós-Graduação } \\
\text { em Matemática) -- Instituto de Ciências Matemáticas } \\
\text { e de Computação, Universidade de São Paulo, } 2015 \text {. }\end{array}$ \\
\hline & $\begin{array}{l}\text { 1. Equações diferenciais. 2. Órbitas periódicas. } 3 . \\
\text { Polinômios radiais. } 4 \text {. Método de Newton. } 5 . \\
\text { Demonstrações assistidas por computador. I. Gameiro, } \\
\text { Marcio Fuzeto, orient. II. Título. }\end{array}$ \\
\hline
\end{tabular}


Aos meus pais, Itamar (in memoriam) e Carmem. 



\section{Resumo}

Neste trabalho, apresentamos um método computacional rigoroso para a demonstração de existência de órbitas periódicas de alguns sistemas de equações diferenciais ordinárias com campo autônomo do tipo polinomial. Mostraremos que o problema de encontrar órbitas periódicas para esses sistemas de equações é equivalente a buscar por raízes de certas funções definidas no espaço de Banach das sequências com decaimento algébrico. O método pode ser dividido em duas etapas. Na primeira, buscamos numericamente por soluções periódicas aproximadas. $\mathrm{Na}$ segunda, mostraremos a existência de uma órbita periódica numa vizinhança da curva encontrada numericamente. $\mathrm{O}$ rigor das verificações computacionais é garantido pelo uso de aritimética intervalar. 



\section{Abstract}

In this work, we present a rigorous computational method for proving the existence of periodic orbits of some systems of ordinary differential equations with autonomous vector field of polynomial type. We show that the problem of finding periodic orbits for these systems is equivalent to check for roots of certain functions defined in the Banach space of sequences with algebraic decay. The method can be divided into two steps. First, we seek, numerically, to approximated periodic solutions. Then, we show the existence of a periodic orbit in a neighborhood of the curve numerically found in the previous stage. The accuracy of the computational verifications is guaranteed by the use of interval arithmetic. 



\section{Conteúdo}

$\begin{array}{ll}\text { Introdução } & 13\end{array}$

1 Propriedades Gerais das EDO's $\quad 15$

1.1 Preliminares . . . . . . . . . . . . . . . . . . . . 15

1.2 Existência e Unicidade de Soluções . . . . . . . . . . . . . . . . . . . 17

1.3 Soluções Maximais . . . . . . . . . . . . . . . . . . . . . . 20

1.4 Dependência dos dados Iniciais e dos Parâmetros . . . . . . . . . . . . . 22

1.5 Sistemas Autônomos . . . . . . . . . . . . . . . . . . . . 24

2 Método de Newton e Polinômios Radiais Em Dimensão Finita 27

2.1 Método de Newton . . . . . . . . . . . . . . . . . . . . 27

2.2 Polinômios Radiais . . . . . . . . . . . . . . . . . . . . . . . . . . . 28

3 Soluções Periódicas da Equação de Michelson $\quad 31$

3.1 O Espaço $\Omega^{s} \ldots \ldots \ldots \ldots \ldots$. . . . . . . . . . . . . . . 32

3.2 Representação de Funções por Séries de Fourier . . . . . . . . . . . . . . 35

3.3 Reformulação do Problema . . . . . . . . . . . . . . . . . . 37

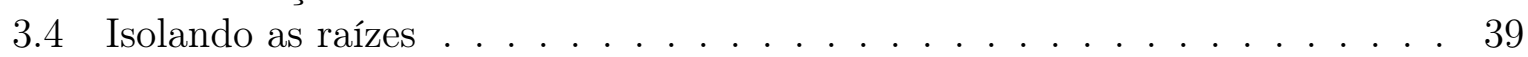

3.5 Redução a um Problema de Ponto fixo . . . . . . . . . . . . . . . . . . . 43

3.5.1 Truncamento . . . . . . . . . . . . . . . 43

3.5.2 Operador de Newton . . . . . . . . . . . . . . . . . . . 44

3.5.3 Construção do operador $A$. . . . . . . . . . . . . . . . 44

3.6 Polinômios Radiais . . . . . . . . . . . . . . . . . . . . . . . . . . 49

3.6.1 Cálculo de $Z(r) \ldots \ldots \ldots \ldots \ldots$. . . . . . . . . . . . . 51

3.6 .2 Cálculo de $Y \ldots \ldots \ldots \ldots \ldots$. . . . . . . . . . . . . 54

3.7 Resultados . . . . . . . . . . . . . . . . . . . 55

4 Soluções Periódicas do Sistema de Lorenz $\quad 59$

4.1 Condição de Fase de Poincaré . . . . . . . . . . . . . . . . . . . . . . . . . 62

4.2 Polinômios radiais para o sistema de Lorenz . . . . . . . . . . . . . . . 65

4.2 .1 Cálculo de $\mathrm{Z}(\mathrm{r}) \ldots \ldots \ldots$. . . . . . . . . . . . . . . . . . 68

4.2 .2 Cálculo de $\mathrm{Y} \ldots \ldots \ldots$. . . . . . . . . . . . . . . . . . 72

4.3 Resultados . . . . . . . . . . . . . . . . . . . . 73

$\begin{array}{lll}5 & \text { Considerações finais } & 79\end{array}$ 


\section{Introdução}

Dentre as muitas utilidades do computador dentro da matemática, uma delas é a capacidade de induzir a formação de certas conjecturas a respeito do objeto de estudo matemático. A abordagem de um problema complexo via simulações numéricas facilita o entendimento da estrutura do problema, mas não constitue-se, por si só, uma demonstração matemática. Sendo assim, é necessário que se desenvolva técnicas capazes de lidar com os resultados retornados pelos métodos numéricos de modo a fornecer conclusões com rigor matemático sobre o problema em estudo.

O método dos polinômios radiais apresentado neste trabalho atua exatamente neste sentido, fornecendo provas matemáticas da existência de certas soluções de sistemas de equações diferenciais ordinárias. Aborda-se aqui, em particular, o estudo da existência de soluções periódicas de sistemas autônomos com campos vetoriais polinomiais. O método prova também a unicidade de órbitas periódicas numa determinada região do espaço de fase.

Em linhas gerais, obtem-se uma reformulação do problema de determinação de órbitas periódicas que condiciona a existência desse tipo de solução à existência de raízes de uma certa função definida num espaço de Banach adequado. O método dos polinômios radiais atua diretamente neste tipo de problema, fornecendo condições de simples verificação computacional rigorosa (com aritmética intervalar) que garantem existência e unicidade local das raízes. Uma variedade de problemas em equações diferenciais é passível deste tipo de reformulação, vide [6], [10], [4]. Sendo assim, os problemas resolvidos aqui são uma pequena fração da gama de posssibilidades de aplicação deste método.

No primeiro capítulo deste trabalho apresentam-se alguns resultados fundamentais em teoria geral de equações diferenciais ordinárias, sem os quais não se faz sentido iniciar uma pesquisa na área. São resultados referentes às condições de existência, unicidade e continuação de soluções, dependência contínua dos dados iniciais e parâmetros e os fatos básicos relacionados aos sistemas autônomos.

No Capítulo 2 apresenta-se brevemente o Método de Newton e uma versão do método dos polinômios radiais em dimensão finita para demonstrar existência de soluções de equilíbrio de campos vetoriais autônomos. Esse capítulo deve ser encarado como motivação para os assuntos tratados nos demais, pois as ideias dele exploram, num nível mais simples e concreto, a estratégia do método dos polinômios radiais.

No Capítulo 3 provamos a existência de órbitas periódicas de uma equação diferencial ordinária não linear de terceira ordem, fornecendo-se todos os resultados e detalhes, tanto técnicos quanto heurísticos, pertinentes à resolução do problema. No Capítulo 4 prova-se a existência de órbitas periódicas para o sistema de Lorenz, aproveitando-se alguns dos resultados obtidos no capítulo anterior. Recorre-se ao pacote MatCont [3] para obtenção de soluções periódicas numéricas desse sistema, em torno das quais pôde-se garantir, via método dos polinômios radiais, a existência e unicidade de uma órbita periódica para o sistema de Lorenz. O método aqui proposto ainda prova a existência de uma curva 
de soluções periódicas, obtida variando-se um dos parâmetros das equações, revelando-se assim parte da dinâmica do sistema.

Sem recorrer ao MatCont obteve-se também uma categoria de soluções periódicas diferente da obtida a partir das órbitas sugeriras pelo pacote MatCont. Essas soluções orbitam os dois pontos de equilíbrio não nulos do sistema, enquanto as sugeridas pelo MatCont envolve apenas um desses pontos.

Cabe ressaltar que todas as computações necessárias ao desenvolvimento deste trabalho foram feitas em MATLAB, e foi imprescindível o uso dos pacotes INTLAB [8], MatCont e da Função fft (fast fourier transform) [1]. O primeiro para garantir o rigor dos resultados das computações, o segundo como simulador de soluções e a Função fft no processo de adaptação dos dados fornecidos pelo MatCont aos moldes do método dos polinômios radiais. 


\section{Capítulo 1}

\section{Propriedades Gerais das EDO's} $[9]$.

Os resultados apresentados neste capítulo podem ser encontrados nas referências [5] e

\subsection{Preliminares}

Iniciamos com um teorema sobre o qual se sustentará grande parte dos resultados apresentados neste trabalho.

Teorema 1.1.1. (Ponto fixo de Banach) Sejam $X$ um espaço de Banach e $C \subset X$ um subconjunto fechado de $X$. Seja $f: C \rightarrow C$ uma função continua para a qual existe $0<\delta<1$ tal que

$$
|f(x)-f(y)| \leq \delta|x-y|, \forall x, y \in C .
$$

Nesse caso, existe um único ponto $\bar{x} \in C$ tal que $f(\bar{x})=\bar{x}$. Além disso, $\bar{x}$ é dado pelo limite da sequência de iterações $\left\{x_{n+1}=f\left(x_{n}\right) ; n \geq 0\right\}$. Mais precisamente, $x_{n} \rightarrow \bar{x}$, independentemente do ponto inicial $x_{0} \in C$ em que começamos as iterações.

Uma função $f$ com as propriedades descritas no enunciado acima é dita uma contração.

Demonstração. Começamos mostrando que $\left\{x_{n}\right\}_{n \geq 0}$ é uma sequência de Cauchy. De fato, podemos escrever

$\left|x_{n}-x_{n-1}\right|=\left|f\left(x_{n-1}\right)-f\left(x_{n-2}\right)\right| \leq \delta\left|x_{n-1}-x_{n-2}\right| \leq \delta^{2}\left|x_{n-2}-x_{n-3}\right| \leq \ldots \leq \delta^{n-1}\left|x_{1}-x_{0}\right|$.

Logo, para $m \geq n$ temos:

$$
\begin{gathered}
\left|x_{m}-x_{n}\right| \leq\left|x_{m}-x_{m-1}\right|+\ldots+\left|x_{n+1}-x_{n}\right| \leq\left(\delta^{m-1}+\ldots+\delta^{n}\right)\left|x_{1}-x_{0}\right|= \\
\delta^{n}\left(1+\delta+\ldots \delta^{m-n}\right)\left|x_{1}-x_{0}\right| \leq \delta^{n} \frac{1}{1-\delta}\left|x_{1}-x_{0}\right| .
\end{gathered}
$$

Como $\delta<1$, dado $\epsilon>0$ podemos obter $n_{0}>0$ tal que

$$
\left|x_{m}-x_{n}\right| \leq \delta^{n} \frac{1}{1-\delta}\left|x_{1}-x_{0}\right| \leq \epsilon ; m \geq n \geq n_{0}
$$

provando que $\left\{x_{n}\right\}_{n \geq 0}$ é uma sequência de Cauchy.

Como $X$ é completo, esta sequência converge para algum $\bar{x} \in X$. Mas, $\left\{x_{n}\right\}_{n \geq 0} \subset C$ e $C$ é fechado. Logo, $\bar{x} \in C$. 
Para ver que $\bar{x}$ é um ponto fixo de $f$ note que

$$
|f(\bar{x})-\bar{x}| \leq\left|f(\bar{x})-f\left(x_{n}\right)\right|+\left|f\left(x_{n}\right)-\bar{x}\right| .
$$

Como $x_{n} \rightarrow \bar{x}$, segue que

$$
\left|f\left(x_{n}\right)-\bar{x}\right|=\left|x_{n+1}-\bar{x}\right| \rightarrow 0 .
$$

Além disso, como $f$ é contínua, segue que

$$
\left|f(\bar{x})-f\left(x_{n}\right)\right| \rightarrow 0 .
$$

Ou seja,

$$
|f(\bar{x})-\bar{x}|=0
$$

Ou ainda,

$$
f(\bar{x})=\bar{x} .
$$

Suponha, por absurdo, que $f$ possua um ponto fixo $\bar{y} \neq \bar{x}$. Nesse caso, podemos escrever:

$$
|\bar{y}-\bar{x}|=|f(\bar{y})-f(\bar{x})| \leq \delta|\bar{y}-\bar{x}|<|\bar{y}-\bar{x}|
$$

o que é absurdo. Logo, $f$ possui exatamente um ponto fixo em $C$.

Observação 1.1.1. O resultado do teorema anterior continua válido ainda se $f$ não for uma contração em $C$, bastando que, para algum natural $m$, sua $m$-ésima iterada $f^{m}=$ $\underbrace{f \circ \ldots \circ f}_{m}$ o seja.

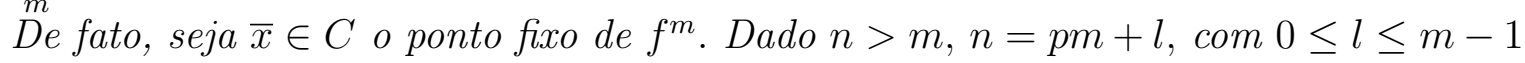
podemos escrever:

$$
\left\|f^{n}\left(x_{0}\right)-f^{m p}\left(x_{0}\right)\right\|=\left\|f^{m p}\left(f^{l}\left(x_{0}\right)\right)-f^{m p}\left(x_{0}\right)\right\| \leq \max _{i=1, \ldots, m-1}\left\{\left\|f^{m p}\left(f^{i}\left(x_{0}\right)\right)-f^{m p}\left(x_{0}\right)\right\|\right\} .
$$

Mas quando $n \rightarrow \infty$, temos que $p \rightarrow \infty$. Se $p \rightarrow \infty$, então, pelo teorema anterior, $f^{m p}\left(f^{i}\left(x_{0}\right)\right) \rightarrow \bar{x}, i=1, \ldots, m-1$ e $f^{m p}\left(x_{0}\right) \rightarrow \bar{x}$. Portanto, $\left\|f^{n}\left(x_{0}\right)-f^{m p}\left(x_{0}\right)\right\| \rightarrow 0$ quando $n \rightarrow \infty$. Como $\left\|f^{n}\left(x_{0}\right)-\bar{x}\right\| \leq\left\|f^{n}\left(x_{0}\right)-f^{m p}\left(x_{0}\right)\right\|+\left\|f^{m p}\left(x_{0}\right)-\bar{x}\right\|$ segue que $f^{n}\left(x_{0}\right) \rightarrow \bar{x}$ qualquer que seja o ponto inicial $x_{0} \in C$. Por fim, note que, definindo $x_{n}=f\left(x_{n-1}\right), n \geq 0$, temos

$$
f(\bar{x})=f\left(\lim x_{n}\right)=\lim f\left(x_{n}\right)=\lim f^{n}\left(x_{0}\right)=\bar{x},
$$

o que demonstra a observação.

A seguir abordamos alguns resultados iniciais em teoria das equações diferenciais ordinárias.

Seja $D$ um subconjunto aberto de $\mathbb{R}^{n+1}$ e $f: D \rightarrow \mathbb{R}^{n}$ contínua. Escrevendo os elementos de $\mathbb{R}^{n+1}$ na forma $(t, x)$, onde $t$ é real e $x \in \mathbb{R}^{n}$, uma equação diferencial ordinária é uma relação da forma

$$
x^{\prime}(t)=f(t, x(t)) .
$$

Dizemos que uma função diferenciável $x: I \rightarrow \mathbb{R}^{n}$, onde $I$ é um intervalo da reta, é solução de (1.1), quando: 
1. $(t, x(t)) \in D, \forall t \in I$

2. $x(t)$ satisfaz a relação (1.1).

Ao subconjunto de $\mathbb{R}^{n}$ onde as soluções $x(t)$ assume valores chamaremos de espaço de fase. À variável $t$ chamaremos tempo e à função $f$ chamaremos campo vetorial da equação diferencial ordinária (1.1).

Dado $\left(t_{0}, x_{0}\right) \in D$, podemos nos perguntar sobre a existência de soluções $x(t)$ de (1.1) que num determinado tempo $t_{0}$ passe por um ponto $x_{0}$. Ou seja:

$$
\left\{\begin{array}{l}
x^{\prime}(t)=f(t, x(t)) \\
x\left(t_{0}\right)=x_{0}
\end{array}\right.
$$

Nos referiremos a este tipo de problema como Problema de Valor Inicial ou, mais sucintamente, PVI.

A princípio, um PVI pode admitir uma, nenhuma ou várias soluções, dependendo do campo vetorial $f$.

Sabe-se que a continuidade do campo vetorial $f$ já garante a existência de soluções para o PVI (1.2), mas não garante a unicidade. Porém, neste trabalho, estamos interessados nos sistemas de equações com unicidade de soluções.

A seguir apresentamos um teorema que dá condições suficientes sobre o campo vetorial $f$ para que ocorra existência e unicidade local de soluções. Com um pouco mais de discussão, mostraremos a unicidade global.

\subsection{Existência e Unicidade de Soluções}

Dizemos que a função $f: D \subset \mathbb{R} \times \mathbb{R}^{n} \rightarrow \mathbb{R}^{n}$ é localmente Lipschitziana com respeito à segunda variável no aberto $D$ se, dado um compacto $U \subset D$, existe $k=k(U)>0$ tal que

$$
\left\|f\left(t, x_{1}\right)-f\left(t, x_{2}\right)\right\| \leq k\left\|x_{1}-x_{2}\right\| \forall\left(t, x_{1}\right), \quad\left(t, x_{2}\right) \in U .
$$

Em particular, se $f \in C^{1}\left(\mathbb{R}^{n+1}, \mathbb{R}^{n}\right)$, decorre da desigualdade do valor médio que $f$ é localmente Lipschitziana. De fato,

$$
\left\|f\left(t, x_{1}\right)-f\left(t, x_{2}\right)\right\| \leq \max _{(\tau, x) \in U}\left\{\left\|f^{\prime}(\tau, x)\right\|\right\}\left\|x_{1}-x_{2}\right\|, \quad\left(t, x_{1}\right), \quad\left(t, x_{2}\right) \in U .
$$

No decorrer deste trabalho denotaremos a bola aberta de centro $a$ e raio $r$ num espaço métrico qualquer por $B(a, r)$. A bola fechada será denotada por $\overline{B(a, r)}$ ou $B[a, r]$.

Teorema 1.2.1. (Existência de Soluções e Unicidade Local) Seja $f$ : D C $\mathbb{R} \times \mathbb{R}^{n} \rightarrow \mathbb{R}^{n}$ continua e localmente Lipschitziana na segunda variável. Dado $\left(t_{0}, x_{0}\right) \in D$, sejam $\alpha>0$ e $\beta>0$ tais que $U=\overline{B\left(t_{0}, \alpha\right)} \times \overline{B\left(x_{0}, \beta\right)} \subset D$, e $M=\max _{x \in U}\|f(x)\|$. Nesse caso, o problema de valor inicial

$$
\left\{\begin{array}{l}
x^{\prime}(t)=f(t, x(t)) \\
x\left(t_{0}\right)=x_{0}
\end{array}\right.
$$

possui única solução da forma

$$
x:\left(t_{0}-\epsilon, t_{0}+\epsilon\right) \rightarrow B\left(x_{0}, \beta\right),
$$


qualquer que seja

$$
0<\epsilon \leq \min \left\{\alpha, \frac{\beta}{M}\right\}
$$

Demonstração. Decorre diretamento do teorema fundamental do cálculo que $x$ é solução do PVI (1.3) se, e somente se,

$$
x(t)=x_{0}+\int_{t_{0}}^{t} f(s, x(s)) d s .
$$

Ou seja, as soluções do PVI (1.3) estão em correspondencia com os pontos fixos do operador $L$ definido no espaço de Banach

$$
C=\left\{x:\left(t_{0}-\epsilon, t_{0}+\epsilon\right) \rightarrow B\left(x_{0}, \beta\right) ; x \text { é contínua e } x\left(t_{0}\right)=x_{0}\right\}
$$

e dado por

$$
L(x)(t)=x_{0}+\int_{t_{0}}^{t} f(s, x(s)) d s, x \in C .
$$

Ou seja, a demonstração do teorema de existência e unicidade é uma aplicação direta do teorema do ponto fixo de Banach e da Observação 1.1.1. Mais precisamente, basta mostrar que $L$ deixa invariante o espaço de Banach $C$ e que $L^{m}$ é uma contração para algum $m>0$.

Dada $x \in C$, é claro que L $(x)$ é contínua e $L(x)\left(t_{0}\right)=x_{0}$. Sendo assim, para ver que $C$ é invariante por $L$ basta observar que $\left\|L(x)(t)-x_{0}\right\|=\left\|\int_{t_{0}}^{t} f(s, x(s)) d s\right\| \leq M \epsilon \leq M \frac{\beta}{M}=\beta$.

Para provar que $L^{m}$ é uma contração para algum natural $m>0$ mostramos, indutivamente, que

$$
\left\|L^{m}(x)(t)-L^{m}(y)(t)\right\| \leq \frac{\delta^{m}\left|t-t_{0}\right|^{m}}{m !}\|x-y\|, x, y \in C
$$

onde $\delta$ é a constante de contração de $f$ no compacto $\left[t_{0}-\epsilon, t_{0}+\epsilon\right] \times B\left[x_{0}, \beta\right]$.

Como $\frac{\delta^{m}\left|t-t_{0}\right|^{m}}{m !} \leq \frac{\delta^{m} \epsilon^{m}}{m !}$ e a série $\sum_{k=0}^{\infty} \frac{\delta^{k} \epsilon^{k}}{k !}$ converge, segue que $\frac{\delta^{k} \epsilon^{k}}{k !} \rightarrow 0$. Logo, existe $m>0$ tal que $\frac{\delta^{m} \epsilon^{m}}{m !}<1$. Para este $m, L^{m}$ é uma contração.

No teorema acima, o intervalo existência e unicidade de soluções depende das condições iniciais. No teorema a seguir, para cada compacto $K \subset D$, obtemos um mesmo intervalo de existência e unicidade válido para todas as condições iniciais dadas por pontos deste compacto.

Corolário 1.2.1. Com as notações do enunciado do teorema anterior, dado um compacto $K \subset D$ existem $\epsilon_{K}>0$ e $b_{K}>0$ tais que, para todo $\left(t_{0}, x_{0}\right) \in K$ o PVI

$$
\left\{\begin{array}{l}
x^{\prime}(t)=f(t, x(t)) \\
x\left(t_{0}\right)=x_{0}
\end{array}\right.
$$

possui única solução definida no intervalo $\left(t_{0}-\epsilon_{K}, t_{0}+\epsilon_{K}\right)$ tomando valores na bola $B\left[x_{0}, b_{K}\right]$ 
Demonstração. Basta encontrar $\alpha>0$ e $\beta>0$ tais que $U\left(t_{0}, x_{0}\right)=B\left[t_{0}, \alpha\right] \times B\left[x_{0}, \beta\right] \subset D$ para todo $\left(t_{0}, x_{0}\right) \in K$ e $M>0$ tal que $\sup _{(t, x) \in U\left(t_{0}, x_{0}\right)}\|f(t, x)\| \leq M$ para todo $\left(t_{0}, x_{0}\right) \in K$.

Feito isso, é só tomar $\epsilon_{K}=\min \left\{\alpha, \frac{\beta}{M}\right\}$.

Seja $K_{1} \subset D$ um compacto contido em $D$ que contém $K$ em seu interior. Seja $M=$ sup $\|f(p)\|$. Para cada $(t, x) \in K$ sejam $a_{t}, b_{x}>0$ tais que $B\left[t, a_{t}\right] \times B\left[x, b_{x}\right] \subset K_{1}$. Seja $p \in K_{1}$

$A=\left\{B\left[t, \frac{a_{t}}{2}\right] \times B\left[x, \frac{b_{x}}{2}\right] ; \quad(t, x) \in K\right\}$ uma cobertura fechada de $K$ e $A_{N}=\left\{B\left[t_{i}, \frac{a t_{i}}{2}\right] \times\right.$ $\left.B\left[x_{i}, \frac{b_{x_{i}}}{2}\right] ;\left(t_{i}, x_{i}\right) \in K, i=1, . ., N\right\}$ uma subcobertura finita de $K$. Defina

$$
a_{m i n}=\min \left\{\frac{b_{x_{i}}}{2} ; i=1, \ldots, N\right\}
$$

e

$$
b_{\min }=\min \left\{\frac{b_{x_{i}}}{2} ; i=1, \ldots, N\right\}
$$

Dado $\left(t_{0}, x_{0}\right) \in K$ tome $i_{0} \in\{1, \ldots, N\}$ tal que $\left(t_{0}, x_{0}\right) \in B\left[t_{i_{0}}, \frac{a_{t_{i_{0}}}}{2}\right] \times B\left[x_{i_{0}}, \frac{b_{x_{i_{0}}}}{2}\right]$. Além disso, $B\left[t_{0}, \frac{a_{\min }}{3}\right] \times B\left[x_{0}, \frac{b_{\min }}{3}\right] \subset B\left[t_{i_{0}}, a_{t_{i_{0}}}\right] \times B\left[x_{i_{0}}, b_{x_{i_{0}}}\right] \subset K_{1} \subset D$. Como $\left(t_{0}, x_{0}\right)$ é qualquer elemento em $K$, segue que

$$
U\left(t_{0}, x_{0}\right)=B\left[t_{0}, \frac{a_{m i n}}{3}\right] \times B\left[x_{0}, \frac{b_{\text {min }}}{3}\right] \subset D, \forall\left(t_{0}, x_{0}\right) \in K
$$

Temos ainda que $\sup _{(t, x) \in U\left(t_{0}, x_{0}\right)}\|f(t, x)\| \leq M$. Logo, basta tomar $\alpha=\frac{a_{\min }}{3}$ e $\beta=\frac{b_{\min }}{3}$. Com isso, definindo $\epsilon_{k}=\min \left\{\alpha, \frac{\beta}{M}\right\}$ e $b_{k}=\beta$, segue, pelo teorema de unicidade local que, para cada $\left(t_{0}, x_{0}\right) \in K$, o PVI

$$
\left\{\begin{array}{l}
x^{\prime}(t)=f(t, x(t)) \\
x\left(t_{0}\right)=x_{0}
\end{array}\right.
$$

admite uma e única solução definida no intervalo $\left(t_{0}-\epsilon_{K}, t_{0}+\epsilon_{K}\right)$ tomando valores na bola $B\left[x_{0}, b_{K}\right]$, como queríamos provar.

Corolário 1.2.2. Se $x_{1}: I_{1} \rightarrow \mathbb{R}^{n}$ e $x_{2}: I_{2} \rightarrow \mathbb{R}^{n}$ são soluções do PVI (1.3) então $x_{1} e$ $x_{2}$ coincidem sobre a interseção $I_{1} \cap I_{2}$ dos intervalos de definição dessas funções.

Demonstração. Seja $\tau=\left\{t \in I_{1} \cap I_{2} ; x_{1}(t)=x_{2}(t)\right\}$. Mostremos que $\tau=I_{1} \cap I_{2}$. Suponha, por absurdo, que $\tau$ é subconjunto próprio de $I_{1} \cap I_{2}$. Note que $\tau=g^{-1}(0)$, onde $g$ é a função contínua $g(t)=x_{1}(t)-x_{2}(t), t \in I_{1} \cap I_{2}$. Logo, $\tau$ é um subconjunto fechado da reta. E, portanto, $\tau$ é um subconjunto próprio e fechado de $I_{1} \cap I_{2}$. Porém, pelo teorema anterior, decorre que $\tau$ também é um aberto da reta. De fato, tome $t_{0} \in \mathrm{e} x_{0}=x_{1}\left(t_{0}\right)=x_{2}\left(t_{0}\right)$. Dados $\alpha, \beta>0$ tais que $U=\overline{B\left(t_{0}, \alpha\right)} \times \overline{B\left(x_{0}, \beta\right)} \subset D$ e $M=\max _{x \in U}\|f(x)\|$, tomamos $\epsilon_{1} \leq \min \left\{\alpha, \frac{\beta}{M}\right\}$. Como $x_{1}\left(t_{0}\right)=x_{0}$ e $x_{2}\left(t_{0}\right)=x_{0}$ por continuidade de $x_{1}$ e $x_{2}$, existe $\epsilon_{2}>0$ tal que $x_{i}(t) \in B\left(x_{0}, \beta\right), t \in\left(t_{0}-\epsilon_{2}, t_{0}+\epsilon_{2}\right) ; i=1,2$.

Tomando $=\min \{1,2\}$, concluímos que $\left.x_{1}\right|_{\left(t_{0}-\epsilon, t_{0}+\epsilon\right)}:\left(t_{0}-\epsilon, t_{0}+\epsilon\right) \rightarrow B\left(x_{0}, \beta\right) \mathrm{e}$ $\left.x_{2}\right|_{\left(t_{0}-\epsilon, t_{0}+\epsilon\right)}:\left(t_{0}-\epsilon, t_{0}+\epsilon\right) \rightarrow B\left(x_{0}, \beta\right)$ são soluções do PVI (1.3), e pelo teorema de unicidade local seque que $\left.\left.x_{1}\right|_{\left(t_{0}-\epsilon, t_{0}+\epsilon\right)} \equiv x_{2}\right|_{\left(t_{0}-\epsilon, t_{0}+\epsilon\right)}$. Ou seja, $\left(t_{0}-\epsilon, t_{0}+\epsilon\right) \subset \tau$. Provamos assim, que $\tau$ e um subconjunto aberto e fechado do intervalo $I_{1} \cap I_{2}$. Por conexidade, segue que $\tau=I_{1} \cap I_{2}$. 


\subsection{Soluções Maximais}

Dizemos que uma solução $\phi(t): I \rightarrow \mathbb{R}^{n}$ do PVI (1.3) é uma solução maximal quando a única solução deste PVI definida num intervalo que contém $I$ é a própria $\phi$. Ou seja, $\phi$ possui o maior domínio de definição que uma solução do PVI (1.3) pode ter.

Seja $f$ como no Teorema 1.2.1. Dado $\left(t_{0}, x_{0}\right) \in D$ denote por $\Psi_{\left(t_{0}, x_{0}\right)}$ o conjunto de todas as soluções do PVI dado em (1.3). Seja $I=\cup_{\phi \in \Psi_{\left(t_{0}, x_{0}\right)}} I_{\phi}$ a união de todos os intervalos de definição das funções do conjunto $\Psi_{\left(t_{0}, x_{0}\right)}$. Como todos esses intervalos tem o ponto $t_{0}$ em comum e todos eles são conexos, segue que $I$ também é conexo, ou seja, $I$ é um intervalo e contém o ponto $t_{0}$. Para cada $t \in I$ escolha $\phi \in \Psi_{\left(t_{0}, x_{0}\right)}$ que contenha $t$ em seu domínio de definição, ou seja, $t \in I_{\phi}$, e defina

$$
\Phi(t)=\phi(t), t \in I_{\phi}
$$

Pelo Corolário 1.2.1 $\Phi$ está bem definida em $I$. Além disso, $\Phi$ é, por construção, solução do PVI (1.3) e, qualquer outra solução tem seu domínio de definição contido em $I$. Ou seja, o PVI (1.3) possui solução maximal, qualquer que seja $\left(t_{0}, x_{0}\right) \in D$. Decorre do Corolário 1.2.1 que esta solução maximal é única.

Por fim, mostremos que o intervalo $I$ da solução maximal tem de ser aberto. Mostraremos que $I$ é aberto à direita. A abertura à esquerda demonstra-se analogamente.

Seja $\Phi: I \rightarrow \mathbb{R}^{n}$ a solução maximal do PVI (1.3). Suponha, por absurdo, que $I$ é fechado à direita com elemento máximo igual a $b$. Por definição de solução, segue que $(b, \Phi(b)) \in D$. Como $D$ é aberto, podemos tomar $\alpha, \beta>0$ tais que $U=\overline{B\left[t_{0}, \alpha\right]} \times \overline{B\left[x_{0}, \beta\right]}$ é um compacto contido em $D$. Pelo Corolário 1.2.1 podemos tomar $\epsilon_{U}>0$ tal que o PVI

$$
\left\{\begin{array}{l}
x^{\prime}(t)=f(t, x(t)) \\
x(b)=\Phi(b)
\end{array}\right.
$$

admite uma e única solução $\xi(t)$ definida no intervalo $\left(b-\epsilon_{U}, b+\epsilon_{U}\right)$. Logo, a função

$$
g(t)=\left\{\begin{array}{l}
\Phi(t), t \leq b \\
\xi(t), t \in\left(b, b+\epsilon_{U}\right)
\end{array}\right.
$$

é uma solução do PVI

$$
\left\{\begin{array}{l}
x^{\prime}(t)=f(t, x(t)) \\
x\left(t_{0}\right)=x_{0}
\end{array}\right.
$$

definida num intervalo que contém propriamente o intervalo $I$ maximal de existência e soluções deste PVI, o que é um absurdo. Logo, I não é fechado à direita. Analogamente prova-se que não é fechado à esquerda.

Teorema 1.3.1. Seja

$$
\Phi:\left(\omega^{-}\left(t_{0}, x_{0}\right), \omega^{+}\left(t_{0}, x_{0}\right)\right) \longrightarrow \mathbb{R}^{n}
$$

a solução maximal do PVI (1.3), e considere um compacto qualquer $K \subset D$. Nessas condições, existem $t_{K}^{+}, t_{K}^{-} \in\left(\omega^{-}\left(t_{0}, x_{0}\right), \omega^{+}\left(t_{0}, x_{0}\right)\right)$ tais que

$$
(t, \Phi(t)) \notin K, t_{K} \leq t<\omega^{+}\left(t_{0}, x_{0}\right)
$$

$e$

$$
(t, \Phi(t)) \notin K, \omega^{-}\left(t_{0}, x_{0}\right)<t \leq t_{K}^{-} .
$$


Demonstração. Suponha o contrário, ou seja, que exista uma sequência $t_{n} \rightarrow \omega^{+}\left(t_{0}, x_{0}\right)$, tal que $\left(t_{n}, \Phi\left(t_{n}\right)\right) \in K, \forall n \geq 0$. Tome uma subsequência convergente $\left(t_{n}^{\prime}, \Phi\left(t_{n}^{\prime}\right)\right) \rightarrow$ $\left(\omega^{+}\left(t_{0}, x_{0}\right), z_{0}\right) \in K$.

Sejam $\epsilon>0$ e $\beta>0$ tais que existe única solução $x:\left[\omega^{+}\left(t_{0}, x_{0}\right)-\epsilon, \omega^{+}\left(t_{0}, x_{0}\right)+\epsilon\right] \longrightarrow$ $B\left[z_{0}, \beta\right]$ do PVI

$$
\left\{\begin{array}{l}
x^{\prime}=f(t, x) \\
x\left(\omega^{+}\left(t_{0}, x_{0}\right)\right)=z_{0}
\end{array} .\right.
$$

Defina $U=\left[\omega^{+}\left(t_{0}, x_{0}\right)-\epsilon, \omega^{+}\left(t_{0}, x_{0}\right)+\epsilon\right] \times B\left[z_{0}, \beta\right]$. Tome $n$ suficientemente grande, de modo que $\left|\omega^{+}\left(t_{0}, x_{0}\right)-t_{n}^{\prime}\right| \leq \epsilon / 3$ e $\left\|z_{0}-\Phi\left(t_{n}^{\prime}\right)\right\| \leq \epsilon / 3$. Defina

$$
U_{1}=B\left[\omega^{+}\left(t_{0}, x_{0}\right), \epsilon / 3\right] \times B\left[z_{0}, \beta / 3\right] \subset U .
$$

Dessa forma, o compacto $U_{2}=B\left[\omega^{+}\left(t_{0}, x_{0}\right), 2 \epsilon / 3\right] \times B\left[z_{0}, 2 \beta / 3\right]$ é tal que $U_{1} \subset U_{2} \subset U$ e existe única única solução do PVI

$$
\left\{\begin{array}{l}
x^{\prime}=f(t, x) \\
x\left(\omega^{+}\left(t_{0}, x_{0}\right)\right)=z_{0}
\end{array}\right.
$$

a qual está definida em $t_{n}^{\prime}+2 \epsilon / 3>\omega^{+}\left(t_{0}, x_{0}\right)$. Como $x$ coincide com $\Phi$ em $\left(t_{n}^{\prime}, \omega^{+}\left(t_{0}, x_{0}\right)\right)$, temos em mãos uma extensão de $\Phi$ a um intervalo maior que o maximal, o que é absurdo.

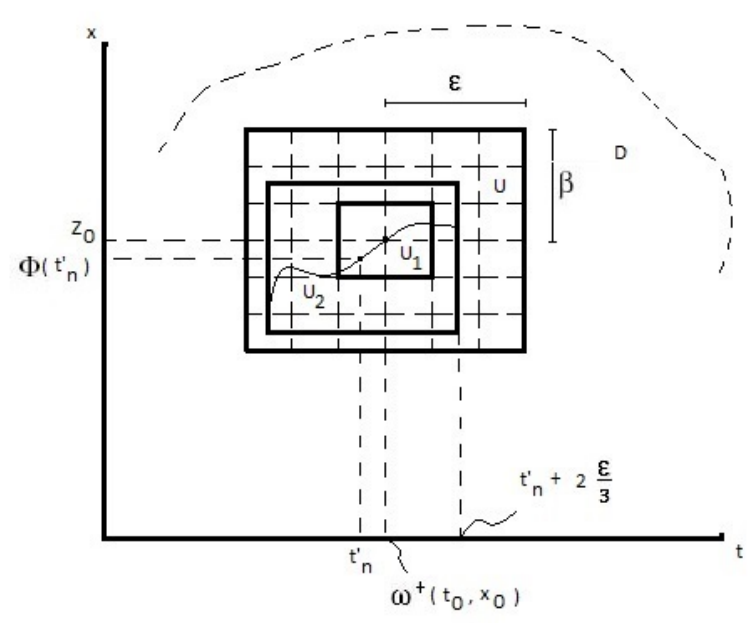

Figura 1.1: Ilustração do raciocíno da demonstração

Sintetizamos os resultados vistos até agora no seguinte teorema, que fornece propriedades introdutórias e essenciais no estudo das soluções de um sistema de equações ordinárias.

Teorema 1.3.2. (Existência e Unicidade Global) Seja $f: D \subset \mathbb{R} \times \mathbb{R}^{n} \rightarrow \mathbb{R}^{n}$ continua e localmente Lipschitziana na segunda variável. Dado $\left(t_{0}, x_{0}\right) \in D$, o problema de valor inicial

$$
\left\{\begin{array}{l}
x^{\prime}(t)=f(t, x(t)) \\
x\left(t_{0}\right)=x_{0}
\end{array}\right.
$$


possui uma e única solução maximal

$$
\Phi:\left(\omega^{-}\left(t_{0}, x_{0}\right), \omega^{+}\left(t_{0}, x_{0}\right)\right) \rightarrow D
$$

onde $\omega^{-}\left(t_{0}, x_{0}\right) \geq-\infty$ e $\omega^{+}\left(t_{0}, x_{0}\right) \leq \infty$.

Além disso, dado um compacto qualquer $K \subset D$, existem $t_{K}^{+}, t_{K}^{-} \in\left(\omega^{-}\left(t_{0}, x_{0}\right), \omega^{+}\left(t_{0}, x_{0}\right)\right)$ tais que

$$
(t, \Phi(t)) \notin K, t_{K} \leq t \leq \omega^{+}\left(t_{0}, x_{0}\right)
$$

$e$

$$
(t, \Phi(t)) \notin K, \omega^{-}\left(t_{0}, x_{0}\right) \leq t \leq t_{K}^{-}
$$

\subsection{Dependência dos dados Iniciais e dos Parâmetros}

Além da existência e unicidade de soluções de um PVI, podemos nos perguntar como se dá a dependência destas soluções com relação às condições iniciais. A proposição 1.4.1 aponta nesta direção. Para demonstrarmos essa proposição precisaremos do seguinte teorema, que, assim como o teorema do ponto fixo de Banach, possui grande número de aplicações na teoria de equações diferenciais. Sua demonstração pode ser encontrada em $[7]$.

Teorema 1.4.1. Arzelà-Ascoli. Seja $(M, d)$ um espaço métrico compacto e $F$ uma família de funções contínuas definidas em $M$ e tomando valores em $\mathbb{R}^{n}$ que satisfaz as seguintes condições:

1. F é equicontínua. Ou seja, dado $\epsilon>0$, existe $\delta>0$ tal que, para quaisquer $x, y \in M$

$$
d(x, y)<\delta \Rightarrow\|\phi(x)-\phi(y)\| \leq \epsilon, \forall \phi \in F .
$$

2. F é uniformemente limitada. Ou seja, existe $K>0$ tal que $\|\phi(x)\| \leq K, \phi \in$ $F, x \in M$.

Sob essas condições, toda sequência de funções $\left\{\phi_{n}\right\}_{n \geq 0} \subset F$ possui uma subsequência que converge uniformemente em $M$ (não necessariamente para uma função pertencente a $F)$.

Proposição 1.4.1. Sejam $D \subset \mathbb{R} \times \mathbb{R}^{q}$ aberto e $f_{n}: D \rightarrow \mathbb{R}^{q}$ uma sequência de funções continuas localmente lipshitzianas na segunda variável e que converge uniformemente para $f_{0}$ em cada compacto de $D$.

Suponha que para cada $n \geq 0$ o PVI

$$
\left\{\begin{array}{l}
x^{\prime}(t)=f_{n}(t, x(t)) \\
x\left(t_{n}\right)=x_{n}
\end{array},\right.
$$

possua única solução maximal dada por

$$
\phi_{n}:\left(\omega^{-}\left(t_{n}, x_{n}\right), \omega^{+}\left(t_{n}, x_{n}\right)\right) \rightarrow \mathbb{R}^{q} .
$$

Nessas condições, dado um intervalo $[a, b] \subset\left(\omega^{-}\left(t_{0}, x_{0}\right), \omega^{+}\left(t_{0}, x_{0}\right)\right)$, existe $n_{0} \in \mathbb{N}$ tal que $\phi_{n}$ está definida pelo menos no intervalo $[a, b]$ para todo $n \geq n_{0}$ e, além disso, a sequência $\left\{\phi_{n}\right\}_{n \geq n_{0}}$ converge uniformemente para $\phi_{0}$ em $[a, b]$. 
Demonstração. Sejam $K$ um compacto contido em $D$ que contém o conjunto $\left\{\left(t, \phi_{0}(t)\right) ; t \in\right.$ $[a, b]\}$ e $M>0$ tais que $\sup _{(t, x) \in K}\left\|f_{0}(t, x)\right\| \leq M$. Como $f_{n} \rightarrow f_{0}$ uniformemente em $K$, existe $n_{0}>0$ tal que $\sup _{(t, x) \in K}\left\|f_{n}(t, x)\right\| \leq M$ para todo $n \geq n_{0}$. Com isso, de acordo com o Corolário 1.2.1, existem $\epsilon_{K}>0$ e $b_{k}>0$ tais que todos os PVI's da forma

$$
\left\{\begin{array}{l}
x^{\prime}=f_{n}(t, x(t)) \\
x(\tau)=\eta
\end{array}\right.
$$

com $n \geq 0$ e $(\tau, \eta) \in K$, possuem soluções definidas pelo menos no intervalo fechado $\left[\tau-\epsilon_{K}, \tau+\epsilon_{K}\right]$.

Tome $0<\alpha \leq \frac{\epsilon_{K}}{2}$ e $0<\beta \leq b_{K}$ tais que $B\left[t_{0}, \alpha\right] \times B\left[x_{0}, \beta\right] \subset K$. Seja $n_{1} \geq n_{0}$ tal que $\left(t_{n}, x_{n}\right) \in B\left[t_{0}, \alpha\right] \times B\left[x_{0}, \beta\right]$ para todo $n \geq n_{1}$. Note que, para todo $n \geq n_{1}$, todas as soluções $\phi_{n}$ estão definidas em $\left[t_{n}-\epsilon_{K}, t_{n}+\epsilon_{K}\right] \supset\left[t_{0}-\frac{\epsilon_{K}}{2}, t_{0}+\frac{\epsilon_{K}}{2}\right], \forall n \geq n_{1}$.

Provemos que as restrições de $\left\{\phi_{n}\right\}_{n \geq n_{1}}$ ao intervalo $\left[t_{0}-\frac{\epsilon_{K}}{2}, t_{0}+\frac{\epsilon_{K}}{2}\right]$ satisfazem as condições do teorema de Arzelà-Ascoli. Para isso, escevemos cada $\phi_{n}$ na forma integral

$$
\phi_{n}(t)=x_{n}+\int_{t_{n}}^{t} f_{n}\left(s, \phi_{n}(s)\right) d s, t \in\left[t_{0}-\frac{\epsilon_{K}}{2}, t_{0}+\frac{\epsilon_{K}}{2}\right] .
$$

Desse modo, podemos ver que

$$
\left\|\phi_{n}\left(t_{2}\right)-\phi_{n}\left(t_{1}\right)\right\| \leq M\left|t_{2}-t_{1}\right|, \forall t_{1}, t_{2} \in\left[t_{0}-\frac{\epsilon_{K}}{2}, t_{0}+\frac{\epsilon_{K}}{2}\right] .
$$

Disso segue que as restrições das funções $\left\{\phi_{n}\right\}_{n \geq n_{1}}$ ao intervalo $\left[t_{0}-\frac{\epsilon_{K}}{2}, t_{0}+\frac{\epsilon_{K}}{2}\right]$ formam uma família equicontínua de funções definidas num compacto. Esta família é uniformemente limitada, pois todos seus elementos tomam valores no compacto $K$. Logo, pelo teorema de Arzelà-Ascoli, toda subsequência desta família converge uniformemente em $\left[t_{0}-\frac{\epsilon_{K}}{2}, t_{0}+\frac{\epsilon_{K}}{2}\right]$. Podemos ver ainda que todas essas subsequências possuem o mesmo limite. De fato, se $\phi_{n^{\prime}} \rightarrow \phi$ uniformemente, escrevendo na forma integral, temos:

$$
\phi_{n^{\prime}}(t)=x_{n^{\prime}}+\int_{t_{n^{\prime}}}^{t} f_{n^{\prime}}\left(s, \phi_{n^{\prime}}(s)\right) d s, t \in\left[t_{0}-\frac{\epsilon_{K}}{2}, t_{0}+\frac{\epsilon_{K}}{2}\right] .
$$

Como $f$ converge uniformemente em $\mathrm{K}$ para $f$, fazendo $n \rightarrow \infty$, obtemos

$$
\phi(t)=x_{0}+\int_{t_{0}}^{t} f(s, \phi(s)) d s, t \in\left[t_{0}-\frac{\epsilon_{K}}{2}, t_{0}+\frac{\epsilon_{K}}{2}\right] .
$$

Mas, por unicidade, segue que $\phi=\phi_{0}$. Ou seja, todas as subsequências de $\phi_{n}$ convergem uniformemente para $\phi_{0}$ em $\left[t_{0}+\frac{\epsilon_{K}}{2}, t_{0}+\frac{\epsilon_{K}}{2}\right]$. Donde concluímos que a própria sequência $\phi_{n}$ converge uniformemente para $\phi_{0}$ em $\left[t_{0}+\frac{\epsilon_{K}}{2}, t_{0}+\frac{\epsilon_{K}}{2}\right]$

Com isso, podemos tomar $n_{2} \geq n_{1}$ tal que $\left(t_{0}+\frac{\epsilon_{K}}{2}, \phi_{n}\left(t_{0}+\frac{\epsilon_{K}}{2}\right)\right) \in K$ para todo $n \geq n_{2}$.

Repetindo o raciocínio acima para o ponto $\left(t_{0}+\frac{\epsilon_{K}}{2}, \phi_{0}\left(t_{0}+\frac{\epsilon_{K}}{2}\right)\right)$ e a sequência de pontos $\left\{\left(t_{0}+\frac{\epsilon_{K}}{2}, \phi_{n}\left(t_{0}+\frac{\epsilon_{K}}{2}\right)\right)\right\}_{n \geq_{2}}$ obteremos $n_{3} \geq n_{2}$ tal que $\phi_{n}$ está definida em $\left[t_{0}+2 \frac{\epsilon_{K}}{2}\right]$ para todo $n \geq n_{3}$ e a sequência $\phi_{n}, n \geq n_{3}$ converge uniformemente para $\phi_{0}$ em $\left[t_{0}+2 \frac{\epsilon_{K}}{2}\right]$.

Repetindo esse processo um número finito de vezes, obtemos o resultado do teorema. 
Suponha agora que o campo vetorial $f$ dependa de um parâmetro $\lambda$ que pode assumir valores num aberto $\Lambda \subset \mathbb{R}^{p}$. Ou seja, $f$ é da forma $f(t, x, \lambda)$ e, nesse caso, obtemos uma família de equações diferenciais ordinárias:

$$
x^{\prime}(t)=f(t, x(t), \lambda), \lambda \in \Lambda
$$

Dado $\left(t_{0}, x_{0}, \lambda_{0}\right) \in D \times \Lambda$, denotaremos por $x\left(\cdot, t_{0}, x_{0}, \lambda_{0}\right)$ a solução do PVI

$$
\left\{\begin{array}{l}
x^{\prime}(t)=f\left(t, x(t), \lambda_{0}\right) \\
x\left(t_{0}\right)=x_{0}
\end{array}\right.
$$

Denotamos o dominio maximal de definição de uma solução $x\left(\cdot, t_{0}, x_{0}, \lambda_{0}\right)$ por $I_{\left(t_{0}, x_{0}, \lambda_{0}\right)}$. Com essas definições em mente, considere a aplicação

$$
x:\left(t, t_{0}, x_{0}, \lambda_{0}\right) \mapsto x\left(t, t_{0}, x_{0}, \lambda_{0}\right) .
$$

Chamaremos a aplicação assim definida por aplicação solução. Note que o domínio $S$ da aplicação $x$ é composto pelos pontos $\left(t, t_{0}, x_{0}, \lambda_{0}\right) \in \mathbb{R} \times D \times \Lambda$ tais que $t \in I_{\left(t_{0}, x_{0}, \lambda_{0}\right)}$.

É consequência da Proposição 1.4.1 que a aplicação solução possui domínio $S$ aberto e é uma aplicação contínua em $S$. Mais precisamente, temos o seguinte corolário.

Corolário 1.4.1. (Dependência Contínua dos Dados Iniciais e Parâmetros) Com as notações definidas acima em mente, sejam $D \subset \mathbb{R} \times \mathbb{R}^{n} e \Lambda \subset \mathbb{R}^{p}$ abertos e $f: D \times \Lambda \rightarrow \mathbb{R}^{n}$ contínua e localmente Lipschitziana na segunda variável. Dado $\left(t_{0}, x_{0}, \lambda_{0}\right) \in D \times \Lambda$, considere uma solução $x\left(t, t_{0}, x_{0}, \lambda_{0}\right)$ do PVI

$$
\left\{\begin{array}{l}
x^{\prime}(t)=f\left(t, x(t), \lambda_{0}\right) \\
x\left(t_{0}\right)=x_{0}
\end{array},\right.
$$

e um intervalo $[a, b] \subset I_{\left(t_{0}, x_{0}, \lambda_{0}\right)}$.

Nessas condições, podemos tomar vizinhanças $B\left(t_{0}, \alpha\right) \times B\left(x_{0}, \beta\right)$ de $\left(t_{0}, x_{0}\right)$ e $B\left(\lambda_{0}, \delta\right)$ de $\lambda_{0}$, de modo que, para todo $(\tau, \eta, \lambda) \in B\left(t_{0}, \alpha\right) \times B\left(x_{0}, \beta\right) \times B\left(\lambda_{0}, \delta\right)$ a solução $x(t, \tau, \eta, \lambda)$ está definida em todo $t \in[a, b]$. Consequentemente, $(a, b) \times B\left(t_{0}, \alpha\right) \times B\left(x_{0}, \beta\right) \times B\left(\lambda_{0}, \delta\right) \subset$ $S$, ou seja, $S$ é aberto.

Além disso, dado $\epsilon>0$ podemos reduzir os raios $\delta, \alpha$ e $\beta$ de modo que

$$
\sup _{t \in[a, b]}\left\|x(t, \tau, \eta, \lambda)-x\left(t, t_{0}, x_{0}, \lambda_{0}\right)\right\| \leq \epsilon
$$

para todo $(\tau, \eta, \lambda) \in B\left(t_{0}, \alpha\right) \times B\left(x_{0}, \beta\right) \times B\left(\lambda_{0}, \delta\right)$. Consequentemente, $x$ é contínua em $\left(\theta, t_{0}, x_{0}, \lambda_{0}\right)$ para todo $\theta \in(a, b)$.

\subsection{Sistemas Autônomos}

Neste trabalho estaremos interessados em estudar equações da seguinte forma

$$
x^{\prime}(t)=f(x(t))
$$

em que o campo vetorial é uma função independente do tempo $t$, definida em um subconjunto do espaço de fase do problema. Este tipo de equação é chamada de equação autônoma. 
O objetivo dessa seção é apresentar algumas definições pertinentes e particularidades que este tipo de equação apresenta.

Em primeiro lugar, note que podemos olhar para $f$ como uma função definida em $\mathbb{R} \times D$ que independe da primeira variável. Ou seja, fazemos $f(t, x)=f(x), t \in \mathbb{R}, x \in D$. Nesse sentido, dado um campo vetorial $f: D \subset \mathbb{R}^{n} \rightarrow \mathbb{R}^{n}$ localmente lipshitziano, $x_{0} \in D$, e $t_{0} \in \mathbb{R}$, os PVI's da forma:

$$
\left\{\begin{array}{l}
x^{\prime}(t)=f(x(t)) \\
x\left(t_{0}\right)=x_{0}
\end{array}\right.
$$

gozam das mesmas propriedades daqueles estudados nas seções anteriores.

A órbita $\Gamma_{\gamma}$ de uma solução $\gamma(t)$ da equação (1.8) é definida como sendo a curva contida no espaço de fase dada por $\left.\Gamma_{\gamma}=\left\{\gamma(t) ; t \in\left(\omega^{-}, \omega^{+}\right)\right\}\right)$, onde $\left(\omega^{-}, \omega^{+}\right)$é o domínio maximal de definição de $\gamma(t)$.

Teorema 1.5.1. Sejam $\gamma_{1}(t)$ e $\gamma_{2}(t)$ soluções maximais da equação (1.8) com dominios maximais de existência dados por $I_{1}$ e $I_{2}$, respectivamente. Suponha que existam $t_{1} \in I_{1}$ e $t_{2} \in I_{2}$ tais que $\gamma_{1}\left(t_{1}\right)=\gamma_{2}\left(t_{2}\right)=p$, ou seja, as órbitas das soluções $\gamma_{1}$ e $\gamma_{2}$ se cruzam no ponto $p$ do espaço de fase.

Nessas condições, as órbitas das soluções $\gamma_{1}$ e $\gamma_{2}$ coincidem e $I_{1}=I_{2}-\left(t_{2}-t_{1}\right)$.

Demonstração. Note que $\gamma_{2}\left(t+t_{2}-t_{1}\right)$, assim como $\gamma_{1}(t)$, é solução do PVI

$$
\left\{\begin{array}{l}
x^{\prime}=f(x) \\
x\left(t_{1}\right)=p
\end{array}\right.
$$

Logo, pelo teorema de unicidade de soluções maximais, segue que $\gamma_{2}\left(t+t_{2}-t_{1}\right)$ possui intervalo maximal de definição $I_{1}$, no qual temos a igualdade $\gamma_{2}\left(t+t_{2}-t_{1}\right)=\gamma_{1}(t)$. Com isso, mostremos que o intervalo maximal de definição $I_{2}$ de $\gamma_{2}$ é dado por

$$
\left\{t+\left(t_{2}-t_{1}\right), t \in I_{1}\right\}=I_{1}+\left(t_{2}-t_{1}\right) .
$$

Como $\gamma_{2}\left(t+t_{2}-t_{1}\right)$ está definida para todo $t \in I_{1}$, é claro que $t+\left(t_{2}-t_{1}\right) \in I_{2}$ para todo $t \in I_{1}$. Ou seja, $I_{1}+\left(t_{2}-t_{1}\right) \subset I_{2}$. Suponha que esta inclusão seja própria. Nesse caso, poderíamos obter um intervalo $I \supset I_{1}$ tal que $\gamma_{2}$ estaria definida em $I+\left(t_{2}-t_{1}\right)$. Ou seja, poderíamos definir $\gamma_{2}\left(t+t_{2}-t_{1}\right)$ em todo o intervalo $I$. Como $I \supset I_{1}$, temos um absurdo, pois $I_{1}$ é o domínio maximal de definição de $\gamma_{2}\left(t+t_{2}-t_{1}\right)$.

Observação 1.5.1. Se $\phi(t):=\phi\left(t, t_{0}, x_{0}\right)$ é uma solução do PVI (1.9), com dominio maximal de definição $\left(\omega^{-}, \omega^{+}\right)$, observe que $\phi\left(t+t_{0}\right)$ é exatamente a solução do PVI,

$$
\left\{\begin{array}{l}
x^{\prime}(t)=f(x(t)) \\
x(0)=x_{0}
\end{array}\right.
$$

com dominio maximal de definição dado por $\left(\omega^{-}-t_{0}, \omega^{+}-t_{0}\right)$. Observe ainda que as órbitas correspondentes às soluçôes dos PVI's (1.9) e (1.10) coincidem.

Logo, poderíamos estudar apenas os PVI's da forma (1.10), ou seja, com condição inicial em $t_{0}=0$.

Considere a equação (1.8) e denote por $\varphi(t, p)$ a solução maximal desta equação que passa pelo ponto $p \in D$ no tempo $t=0(\varphi(0, p)=p)$ e por $I_{p}=\left(\omega_{p}^{-}, \omega_{p}^{+}\right)$o domínio maximal dessa solução. Nessas condições, a função $\varphi(t, p)$ está definida num aberto $\Omega \subset \mathbb{R} \times D$ que contém o ponto $(0, p)$ e é contínua em $\Omega$ (Isto segue diretamente do teorema de dependência contínua, visto na seção anterior). 
À aplicação $\varphi: \Omega \rightarrow D$ denominaremos Fluxo da equação autônoma (1.8).

Teorema 1.5.2. (Propriedades do Fluxo) Dado $p \in D$ e $\tau \in I_{p}$, temos que:

- $I_{\varphi(\tau, p)}=I_{p}-\tau$,

- $\varphi(t+\tau, p)$ está definida para todo $t \in I_{\varphi(\tau, p)}$ e

- $\varphi(t+\tau, p)=\varphi(t, \varphi(\tau, p)), \forall t \in I_{\varphi(\tau, p)}$.

Demonstração. Dados $p \in D$ e $\tau \in I_{p}$, defina

$$
\gamma_{1}(t)=\varphi(t, p) \text { e } \gamma_{2}(t)=\varphi(t, \varphi(\tau, p))
$$

Por definição de $\varphi$, sabemos que $\gamma_{1}(t)$ e $\gamma_{2}(t)$ são, respectivamente, as soluções maximais dos PVI's :

$$
\left\{\begin{array} { l } 
{ x ^ { \prime } ( t ) = f ( x ( t ) ) } \\
{ x ( 0 ) = p }
\end{array} \text { e } \quad \left\{\begin{array}{l}
x^{\prime}(t)=f(x(t)) \\
x(0)=\varphi(\tau, p)
\end{array}\right.\right.
$$

Além disso, $\gamma_{1}(\tau)=\gamma_{2}(0)=\varphi(\tau, p)$. Logo, pelo Teorema 1.5.1, concluímos que $\Gamma_{\gamma_{1}}=\Gamma_{\gamma_{2}}$ e $I_{p}=I_{\varphi(\tau, p)}-(0-\tau)$. Ou seja, $I_{\varphi(\tau, p)}=I_{p}-\tau$. De onde segue que a função $\varphi(t+\tau, p)$ está definida para todo $t \in I_{\varphi(\tau, p)}$.

Como $\varphi(0+\tau, p)=\varphi(\tau, p)$ e $\varphi(t+\tau, p)$ está definida em todo o intervalo $I_{\varphi(\tau, p)}$, segue, por unicidade de soluções maximais, que $\varphi(t+\tau, p)$ é solução maximal do PVI

$$
\left\{\begin{array}{l}
x^{\prime}(t)=f(x(t)) \\
x(0)=\varphi(\tau, p)
\end{array}\right.
$$

Ou seja, $\varphi(t+\tau, p)=\varphi(t, \varphi(\tau, p))$ para todo $t \in I_{\varphi(\tau, p)}$, como queríamos provar.

Corolário 1.5.1. Se a curva dada por uma órbita $\Gamma$ de uma solução $\gamma$ da equação (1.8) se autointercepta, então $\Gamma$ é uma curva fechada. Em outras palavras, se houverem reais $t_{1} \neq t_{2}$ tais que $\gamma\left(t_{1}\right)=\gamma\left(t_{2}\right)$, então $\gamma\left(t+t_{1}-t_{2}\right)=\gamma(t)$ para todo $t \in \mathbb{R}$. Nesse caso, $\gamma(t)$ é dita uma solução periódica da equação (1.8). 


\section{Capítulo 2}

\section{Método de Newton e Polinômios Radiais Em Dimensão Finita}

Um ponto $p \in D$ é dito ponto de equilíbrio da equação (1.8) se $p$ é um zero do campo vetorial $f$ desta equação. Ou seja, $f(p)=0$. Em particular, se $p$ é um ponto de equilíbrio de (1.8), então a função $x(t)=p, t \in \mathbb{R}$ é uma solução de (1.8), chamada solução de equilíbrio.

No que segue, descrevemos um método para encontrar numericamente raízes aproximadas de funções da forma $f: D \subset \mathbb{R}^{n} \rightarrow \mathbb{R}^{n}$ e apresentamos uma estratégia de demonstração assistida por computador, que denominaremos método dos polinômios radiais, para garantir a existência de raízes próximas às soluções numéricas obtidas.

Com isso, a estratégia dos polinômios radiais pode ser aplicada na demonstração de existência de soluções de equilíbrio de um sistema de equações diferenciais ordinárias qualquer.

\subsection{Método de Newton}

Considere o problema de determinar raízes de $f: D \subset \mathbb{R}^{n} \rightarrow \mathbb{R}^{n}$. Esse problema é equivalente a encontrarmos pontos fixos de operadores da forma $T(x)=x-A f(x)$ onde $A: \mathbb{R}^{n} \rightarrow \mathbb{R}^{n}$ é um operador linear inversível. De fato, para $\bar{x} \in \Omega$, temos as equilavências:

$$
T(\bar{x})=\bar{x} \Leftrightarrow A f(\bar{x})=0 \Leftrightarrow f(\bar{x})=A^{-1} 0 \Leftrightarrow f(\bar{x})=0 .
$$

A vantagem da abordagem via operador de ponto fixo é que o Teorema do Ponto Fixo de Banach nos dá um método iterativo de determinação desses pontos. Mais precisamente, se o operador $A$ for escolhido de modo que $T$ seja uma contração que deixa o compacto $\Omega$ invariante, ou seja, $T(\Omega) \subset \Omega$, ao iterarmos o operador $T$ a partir de um ponto inicial $x_{0} \in \Omega$ qualquer, a sequência $\left\{T^{n}\left(x_{0}\right)\right\}_{n \geq 1}$, de acordo com o Teorema do Ponto Fixo de Banach, convergirá para um ponto fixo de $T$, ou seja, para uma raiz de $f$. Utilizando um computador para efetuar essas iterações um número suficientemente grande de vezes, teremos uma aproximação para uma raiz de $f$.

Pela desigualdade do valor médio, para todos $x, y \in \Omega$ temos que

$$
|T(x)-T(y)| \leq \sup _{\theta \in \Omega}\|D T(\theta)\||x-y|=\sup _{\theta \in \Omega}\|I d-A D f(\theta)\||x-y| .
$$

Suponha que a derivada de $f$ seja inversível num ponto $x_{0}$. A desigualdade (2.1) motiva a escolha do operador $A$ como sendo a inversa de $D f\left(x_{0}\right)$. De fato, se $A=\left[D T\left(x_{0}\right)\right]^{-1}$, 
temos que $D T\left(x_{0}\right)=0$ e, por continuidade, $\|D T(x)\|<1$ para $x$ suficientemente próximo de $x_{0}$.

Logo, dada uma função $f: D \subset \mathbb{R}^{n} \rightarrow \mathbb{R}^{n}$ para a qual queremos encontrar uma raiz, podemos escolher $x_{0} \in D$ e executarmos computacionalmente as iterações $x_{n+1}=$ $x_{n}-\left[D f\left(x_{0}\right)\right]^{-1} f\left(x_{n}\right)$ na esperança de que as distâncias $\left|x_{n+1}-x_{n}\right|$ fiquem cada vez menores, indicando uma possível convergência. A este processo, denominamos Método de Newton.

Na prática, estipulamos $\epsilon>0$ suficientemente pequeno (da ordem de $10^{-14}$, nos casos estudados nesse trabalho) e executamos as iterações acima até que ocorra $\left|x_{n+1}-x_{n}\right| \leq \epsilon$, e $f\left(x_{n}\right) \approx 0$. Nesse caso, tomamos o último valor de $x_{n}$ retornado por essas iterações como sendo uma aproximação de uma raiz de $f$. Se essa condição de convergência não for verificada após um número máximo de iterações estipulado previamente, assumimos que o método não converge a partir do ponto inicial $x_{0}$ escolhido.

Suponha que tenhamos obtido, computacionalmente, um ponto $\bar{x} \in \mathbb{R}^{n}$ tal que $f(\bar{x}) \approx$ 0. Queremos mostrar que, de fato, numa pequena vizinhança desta aproximação numérica existe uma raiz de $f$. Para isso, na seção seguinte apresentamos um método cujo objetivo é obter uma bola centrada no ponto $\bar{x}$ na qual podemos garantir que o operador de Newton $T$ é uma contração, demonstrando, pelo Teorema do Ponto Fixo de Banach, a existência de uma única raíz de $f$ nessa bola.

\subsection{Polinômios Radiais}

Considere em $\mathbb{R}^{n}$ a norma do máximo, dada por $\left\|\left(x_{1}, \ldots, x_{n}\right)\right\|=\max \left\{\left|x_{1}\right|, . .,\left|x_{n}\right|\right\}$. Seja $f: \mathbb{R}^{n} \rightarrow \mathbb{R}^{n}$ uma função diferenciável, e $\bar{x} \in \mathbb{R}^{n}$ (obtido numericamente) tal que $f(\bar{x}) \approx 0$.

Se $D f(\bar{x})$ é invertível, fixe $A \approx[D f(\bar{x})]^{-1}$ uma matriz inversível que se aproxima da matriz $[D f(\bar{x})]^{-1}$. A matriz $A$ pode ser obtida por qualquer Software matemático. Nesse trabalho, usamos o Software MATLAB. Note que $A \cdot[D f(\bar{x})]^{-1} \approx I d$.

Em seguida, defina o operador de ponto fixo dado por

$$
T(x)=x-A f(x)
$$

Note que, como $A$ é inversível, os pontos fixos de $T$, se houverem, estão em correspondência com as raízes de $f$.

Queremos usar o teorema do ponto fixo de Banach para obter $r>0$ tal que $T$ possua ponto fixo no compacto $B[\bar{x}, r]$. Para isso, precisamos garantir que a bola $B[\bar{x}, r]$ fica invariante por $T$. Ou seja, é necessário que $T(B[\bar{x}, r]) \subset B[\bar{x}, r]$, ou seja, $\|T(x)-\bar{x}\| \leq r$ para todo $x \in B[\bar{x}, r]$. Nesse sentido, temos:

$$
\begin{gathered}
\|T(x)-\bar{x}\| \leq\|T(\bar{x})-\bar{x}\|+\|T(x)-T(\bar{x})\| \leq \\
\quad \leq\|T(\bar{x})-\bar{x}\|+\sup _{\theta \in B[\bar{x}, r]}\|D T(\theta)\|\|x-\bar{x}\| \leq \\
\quad \leq\|T(\bar{x})-\bar{x}\|+\sup _{u, v \in B[0,1]}\|D T(\bar{x}+r u) v\| r .
\end{gathered}
$$

Logo, como estamos considerando a norma do máximo em $\mathbb{R}^{n}$, para que $T(B[\bar{x}, r]) \subset$ $B[\bar{x}, r]$ basta que

$$
\left|T_{k}(\bar{x})-\bar{x}_{k}\right|+\sup _{u, v \in B[0,1]}\left|D T_{k}(\bar{x}+r u) v\right| \cdot r<r, k=1, \ldots, n .
$$


Vejamos que a validade das desigualdades dadas por (2.3) também garante que o operador $T$ é uma contração. De fato, pela desigualdade do valor médio, temos:

$$
\left|T_{k}(x)-T_{k}(y)\right| \leq \sup _{\theta \in B[\bar{x}, r]}\left\|D T_{k}(\theta)\right\|\|x-y\|=\sup _{u, v \in B[0,1]}\left|D T_{k}(\bar{x}+r u) v\right|\|x-y\| .
$$

Decorre das desigualdades dadas por (2.3) que $\sup _{u, v \in B[0,1]}\left|D T_{k}(\bar{x}+r u) v\right| \cdot r<r$, ou seja,

$$
\sup _{u, v \in B[0,1]}\left|D T_{k}(\bar{x}+r u) v\right|<1, k=1, \ldots, n .
$$

Por (2.4) e (2.5), temos que

$$
\left|T_{k}(x)-T_{k}(y)\right| \leq \delta\|x-y\| \text { para algum } \delta<1,
$$

$\log 0$

$$
\|T(x)-T(y)\|=\max _{k \in\{1, \ldots, n\}}\left|T_{k}(x)-T_{k}(y)\right| \leq \delta\|x-y\|,
$$

ou seja, $T$ é uma contração.

Vemos assim que a validade das desigualdades (2.3) garante que $T(x)=x-A f(x)$ é uma contração na bola $B[\bar{x}, r]$, o que implica, pelo teorema do ponto fixo de Banach, que $T(x)$ possui um único ponto fixo na bola $[\bar{x}, r]$, o qual corresponde à unica raiz de $f$ em $B[\bar{x}, r]$.

Suponha que tenhamos encontrado $Y=\left(Y_{1}, \ldots, Y_{n}\right) \in \mathbb{R}^{n}$ e polinômios em $r$ dados por $Z_{1}(r), \ldots, Z_{n}(r)$ tais que

$$
\left|T_{k}(\bar{x})-\bar{x}\right| \leq Y_{k}, k=1, \ldots, n
$$

$\mathrm{e}$

$$
\sup _{u, v \in B[\bar{x}, r]}\left|D T_{k}(\bar{x}+r u) v\right| r \leq Z_{k}(r), k=1, \ldots, n .
$$

Os polinômios

$$
P_{k}(r):=Y_{k}+Z_{k}(r)-r, k=1, \ldots, n
$$

são chamados Polinômios Radiais.

Note que, se houver $r>0$ tal que $P_{k}(r)<0$ para todo $k \in\{1,2, \ldots, n\}$, então as desigualdades em (2.3) são verificadas e, portanto, $f$ possui uma única raiz na bola $B[\bar{x}, r]$.

Sintetizamos essa discussão no seguinte teorema:

Teorema 2.2.1. Seja $f: \mathbb{R}^{n} \rightarrow \mathbb{R}^{n}$ diferenciável e $\bar{x} \in \mathbb{R}^{n}$ tal que $D f(\bar{x})$ é inversível. Seja A uma aproximação numérica para $[D f(\bar{x})]^{-1}$. Suponha que A seja inversível. Seja $Y=\left(Y_{1}, \ldots, Y_{n}\right) \in \mathbb{R}^{n}$ tal que

$$
\left|T_{k}(\bar{x})-\bar{x}\right| \leq Y_{k}, k=1, \ldots, n .
$$

Suponha que tenhamos encontrados polinômios $Z_{1}(r), \ldots, Z_{n}(r)$ tais que

$$
\sup _{u, v \in B[\bar{x}, r]}\left|D T_{k}(\bar{x}+r u) v\right| r \leq Z_{k}(r), k=1, \ldots, n .
$$

Defina os polinômios radiais

$$
P_{k}(r):=Y_{k}+Z_{k}(r)-r, k=1, \ldots, n .
$$

Nessas condições, se houver $r>0$ tal que $P_{k}(r)<0$ para todo $k \in\{1, \ldots, n\}$, então $f$ possui uma única raiz na bola $B[\bar{x}, r]$. 
Uma vez obtidas analiticamente as cotas $Y$ e $Z(r)=\left(Z_{1}(r), \ldots, Z_{n}(r)\right)^{T}$, vejamos como as hipóteses do Teorema 2.2.1 podem ser verificadas de modo rigoroso utilizando o computador.

A princípio, o computador comete erros de arredondamento durante os cálculos aritméticos necessários para a determinação das cotas $Y$ e $Z(r)$. Além disso, as verificações das desigualdades $P_{k}(r)<0$ para algum $r>0$ também estão sujeitas às imprecisões numéricas. É importante notar também que a implementação das cotas $Y$ e $Z(r)$ e as verificações $P_{k}(r)<0$ para algum $r>0$ constituem os únicos cálculos imprescindíveis de rigor matemático.

Para manter o rigor matemático, fazemos todos os cálculos envolvidos na determinação dos polinômios radiais com aritmética de intervalo. Isso quer dizer que todas as variáveis envolvidas nos cálculos são tratadas como intervalos que englobam os erros de arredondamento cometidos pelo computador. A aritmética intervalar executa os cálculos com esses intervalos, retornando um outro intervalo que contém seguramente o resultado exato da operação em questão. Dessa maneira, os coeficientes dos polinômios radiais serão dados por intervalos que contém os coeficientes verdadeiros. Chamaremos esses polinômios de polinômios intervalares e seus coeficientes serão chamados de coeficientes intervalares. Todos os cálculos em aritmética de intervalo realizados nesse trabalho são executados pelo pacote INTLAB [8] do software MATLAB.

Para cada polinômio intervalar obtido acima, consideramos o polinômio cujos coeficientes são dados pelos pontos médios dos coeficientes intervalares. Chamamos os polinômios assim obtidos por polinômios médios. Para esses polinômios, calculamos numericamente $r>0$ para o qual todos os polinômios médios são negativos.

Finalmente, voltamos à aritmética de intervalo para avaliar todos os polinômios intervalares em $r$. O resultado de cada avaliação é um intervalo que contém, particularmente, o resultado exato da avaliação do polinômio correto em $r$. Se todos esses intervalos estiverem contidos na parte negativa da reta real, em particular, todos os polinômios avaliados em $r$ assumirão, seguramente, valores negativos, verificando rigorosamente as hipóteses do Teorema 2.2.1.

Podemos aplicar conjuntamente o Método de Newton e o Teorema 2.2.1 para provarmos resultados simples em equações diferenciais ordinárias, como existência de soluções de equilíbrio.

Nosso principal objetivo neste trabalho é descrever um método, baseado no Método de Newton e na estratégia dos polinômios radiais, para obter aproximações de órbitas periódicas de alguns sistemas de EDO's e, em seguida, provar a existência de órbitas periódicas numa vizinhança dessas aproximações. Analogamente à investigação de soluções de equilíbrio, veremos que é possível condicionar a existência de órbitas periódicas à existência de raízes de funções definidas em espaços de Banach. Assim, precisaremos desenvolver algumas ideias e estimativas que permitem obter condições em dimensões finitas que garantem resultados em dimensões infinitas. Faremos isso nas seções seguintes. 


\section{Capítulo 3}

\section{Soluções Periódicas da Equação de Michelson}

Neste capítulo provaremos a existência de órbitas periódicas para a seguinte equação não linear de terceira ordem para alguns valores do parâmetro $\lambda \in \mathbb{R}$ (Veja [4]).

$$
x^{\prime \prime \prime}+x^{\prime}+\frac{x^{2}}{2}=\lambda^{2} .
$$

Antecipemos aqui, heuristicamente, alguns pontos da abordagem que daremos a este problema, a qual está de acordo com [4].

Se $\gamma(t)$ é uma solução periódica para a equação (3.1), podemos escrevê-la em termos de sua série de Fourier

$$
\gamma(t)=\sum_{k \in \mathbb{Z}} c_{k} e^{-i k l t}
$$

Escrevendo a equação (3.1) em termos desta série obtemos um sistema de infinitas equações algébricas nas variáveis $l, c_{0}, c_{1}, \ldots$ Sintetizamos essas variáveis na nova variável $c=$ $\left\{l, c_{0}, c_{1}, \ldots\right\}$. Provaremos que o conjunto das sequências $c=\left\{l, c_{0}, c_{1}, \ldots\right\}$ de coeficientes de Fourier das soluções periódicas de (3.1) pode ser inserido num determinado espaço de Banach adequado. Com isso, reduzimos o problema do cálculo de órbitas periódicas à resolução de infinitas equações algébricas em infinitas variáveis.

Escrevemos o sistema mencionado acima na forma:

$$
\left\{\begin{array}{l}
F_{0}(c)=0 \\
F_{1}(c)=0 \\
F_{2}(c)=0 \\
\vdots
\end{array}\right.
$$

As soluções desse sistema são exatamente as raízes da função $F$ dada por $F(x)=$ $\left\{F_{k}(x)\right\}_{k=0}^{\infty}$, definida e tomando valores em espaços de Banach que serão descritos em detalhes logo na primeira seção deste capítulo.

Sendo assim, o método proposto nesse trabalho tem como objetivo fornecer (analiticamente) e verificar (computacionalmente) condições suficientes para a existência de raízes dessas funções.

Primeiramente faremos uma busca numérica por soluções periódicas aproximadas de (3.1). Fazemos isso estudando a versão truncada do problema, buscando por soluções aproximadas de (3.1) da forma

$$
\gamma_{m}(t)=\sum_{|k|<m} c_{k} e^{-i k l t} .
$$


Dessa forma, o sistema de equações descrito acima fica restrito a um número finito de equações em finitas variáveis. Porém, dada a dominância dos primeiros coeficientes da série de Fourier sobre o comportamento da solução periódica que por ela é descrita, espera-se encontrar uma raiz aproximada desse sistema (ou, equivalentemente, uma solução periódica aproximada de (3.1)), desde que o truncamento considere uma quantidade significativa de coeficientes. Veremos que essa aproximação poderá ser obtida numericamente via Método de Newton para funções de várias variáveis.

Na sequência, provaremos a existência de raízes de $F$ numa vizinhança da aproximação $\bar{c}$ obtida na primeira etapa. Para isso, construiremos um operador da forma $T(x)=x-A F(x)$, onde $A$ é linear e inversível, de modo que pontos fixos de $T$ correspondam a raízes de $F$. Analiticamente, encontraremos condições que podem ser verificadas pelo computador de maneira rigorosa e que garantem a existência de uma vizinhança da aproximação $\bar{c}$ na qual o operador $T$ é uma contração. Feito isso, ficará garantida, pelo teorema do ponto fixo de Banach, a existência de um ponto fixo de $T$, ou seja, de uma raiz de $F$, na região em que verificamos que $T$ é uma contração. Mais precisamente, mostraremos que, a fim de garantir que o operador $T$ acima é de fato uma contração numa bola $B[\bar{c}, r]$ é suficiente que um número infinito de polinômios de segundo grau assumam valores negativos em $r$.

Como em toda demonstração assistida por computador, precisamos garantir que o método se detenha a uma quantidade finita de computações e que os erros de aproximação cometidos pelo computador sejam superados por algum mecanismo, de modo a eximir qualquer prejuízo ao rigor da demonstração.

A princípio, como observado acima, teremos uma quantidade infinita de condições a serem verificadas computacionalmente (infinitos polinômios retornando valores negativos quando avaliados num determinado número $r$ ). Porém, reduziremos analiticamente essas condições a um número finito de desigualdades.

A fim de garantir precisão computacional dessas verificações trabalharemos sempre com aritmética de intervalo. Nessa abordagem, cada número será considerado como um intervalo, de modo que qualquer operação entre esses números retornará um outro intervalo, o qual, seguramente, conterá o resultado exato das operações aritméticas em questão.

A seguir, descrevemos em detalhes os pontos comentados acima.

\subsection{O Espaço $\Omega^{s}$}

O espaço vetorial descrito nessa seção será o ambiente no qual desenvolveremos o método proposto neste trabalho. Sendo assim, destacamos aqui algumas de suas propriedades.

Apenas para efeito de uniformização da apresentação, definimos:

$$
\omega_{k}=\left\{\begin{array}{ll}
1 & \text { se } k=0 \\
|k| & \text { se }|k|>0
\end{array} \quad k \in \mathbb{Z}\right.
$$

Definição 3.1.1. Para cada número real $s>0$ definimos o conjunto

$$
\Omega^{s}:=\left\{\left(c_{k}\right)_{k=0}^{\infty} \mid c_{k} \in \mathbb{C}^{n}, \sup _{k \geq 0}\left\{\left\|c_{k}\right\|_{\infty} \cdot \omega_{k}^{s}\right\}<\infty\right\}
$$

e a aplicação

$$
\|\cdot\|_{s}: \Omega^{s} \rightarrow \mathbb{R}
$$




$$
\|c\|_{s}=\sup _{k \geq 0}\left\{\left\|c_{k}\right\|_{\infty} \cdot \omega_{k}^{s}\right\}, c \in \Omega^{s}
$$

Teorema 3.1.1. Para todo $s>0$, a aplicação $\|\cdot\|_{s}: \Omega^{s} \rightarrow \mathbb{R}$ é uma norma em $\Omega^{s}$ (norma s). Além disso, o conjunto $\Omega^{s}$, munido das operações de soma termo a termo $\left(c_{k}\right)_{k=0}^{\infty}+\left(d_{k}\right)_{k=0}^{\infty}=\left(c_{k}+d_{k}\right)_{k=0}^{\infty}$ e da multiplicação por escalar $z\left(c_{k}\right)_{k=0}^{\infty}=\left(z c_{k}\right)_{k=0}^{\infty}, z \in \mathbb{C}$, é um espaço vetorial, o qual, munido da norma $\|\cdot\|_{s}$, é um espaço de Banach.

Somatórios do tipo apresentados no teorema a seguir surgirão de modo recorrente neste trabalho e será de suma importância sermos capazes de estimá-los.

Teorema 3.1.2. Dados $s \geq 2$ e $M \geq 6$ existe uma sequência de números positivos $\left\{\sigma_{k}(s)\right\}_{k \geq 0}$ tal que

$$
\sum_{j=0}^{\infty} \omega_{k-j}^{-s} \omega_{j}^{-s} \leq \frac{\sigma_{k}(s)}{\omega_{k}^{s}}, \forall k \geq 0
$$

e, além disso,

$$
\sigma_{k}(s) \leq \sigma_{M}(s), \forall k \geq M
$$

Demonstração. Para $k=0$ temos

$$
\begin{aligned}
& \sum_{j=0}^{\infty} \omega_{k-j}^{-s} \omega_{j}^{-s}=1+\sum_{j=1}^{\infty} \frac{1}{j^{2 s}}<1+2 \cdot 2^{-2 s}+4 \cdot 4^{-2 s}+8 \cdot 8^{-2 s}+\cdots= \\
& =1+2^{-2 s+1}+\left(2^{-2 s+1}\right)^{2}+\left(2^{-2 s+1}\right)^{3}+\cdots=1+\frac{1}{2^{2 s-1}-1}:=\sigma_{0}(s) .
\end{aligned}
$$

Seja $k \geq 1$. Nesse caso, podemos escrever:

$$
\begin{aligned}
\sum_{j=0}^{\infty} \omega_{k-j}^{-s} \omega_{j}^{-s}= & \frac{2}{k^{s}}+\sum_{j=1, j \neq k}^{\infty} \frac{1}{|k-j|^{s} j^{s}}=\frac{2}{k^{s}}+\sum_{j=1}^{k-1} \frac{1}{(k-j)^{s} j^{s}}+\sum_{j=k+1}^{\infty} \frac{1}{(j-k)^{s} j^{s}}= \\
& \frac{2}{k^{s}}+\sum_{j=1}^{k-1}\left[\left(\frac{1}{k-j}+\frac{1}{j}\right) \frac{1}{k}\right]^{s}+\sum_{j=1}^{\infty} \frac{1}{(j+k)^{s} j^{s}}= \\
= & \frac{2}{k^{s}}+\frac{1}{k^{s}} \sum_{j=1}^{k-1}\left(\frac{1}{k-j}+\frac{1}{j}\right)^{s}+\frac{1}{k^{s}} \sum_{j=1}^{\infty}\left(\frac{1}{j}-\frac{1}{k+j}\right)^{s} .
\end{aligned}
$$

Como $s>2$ temos

$$
\sum_{j=1}^{\infty}\left(\frac{1}{j}-\frac{1}{k+j}\right)^{s} \leq \sum_{j=1}^{\infty}\left(\frac{1}{j}\right)^{s} \leq \sum_{j=1}^{\infty}\left(\frac{1}{j}\right)^{2}=\frac{\pi^{2}}{6} .
$$

Por outro lado,

$$
\begin{aligned}
& \sum_{j=1}^{k-1}\left(\frac{1}{k-j}+\frac{1}{j}\right)^{s}=2\left(\frac{k}{k-1}\right)^{s}+\sum_{j=2}^{k-2}\left(\frac{1}{k-j}+\frac{1}{j}\right)^{s}= \\
& =2\left(\frac{k}{k-1}\right)^{s}+\sum_{j=2}^{k-2}\left(\frac{1}{k-j}+\frac{1}{j}\right)^{2}\left(\frac{1}{k-j}+\frac{1}{j}\right)^{s-2} \leq
\end{aligned}
$$




$$
\begin{gathered}
\leq 2\left(\frac{k}{k-1}\right)^{s}+\left(\frac{1}{2}+\frac{1}{k-2}\right)^{s-2} \sum_{j=2}^{k-2}\left(\frac{1}{k-j}+\frac{1}{j}\right)^{2}= \\
=2\left(\frac{k}{k-1}\right)^{s}+\left(\frac{1}{2}+\frac{1}{k-2}\right)^{s-2}\left[\sum_{j=2}^{k-2} \frac{1}{(k-j) 2}+\frac{1}{j^{2}}+\frac{2}{j(k-j)}\right]= \\
=2\left(\frac{k}{k-1}\right)^{s}+\left(\frac{1}{2}+\frac{1}{k-2}\right)^{s-2}\left[2 \sum_{j=2}^{k-2} \frac{1}{j^{2}}+\frac{2}{k} \sum_{j=2}^{k-2}\left(\frac{1}{j}+\frac{1}{k-j}\right)\right]= \\
\leq 2\left(\frac{k}{k-1}\right)^{s}+\left(\frac{1}{2}+\frac{1}{k-2}\right)^{s-2}\left[\frac{\pi^{2}}{3}+\frac{4}{k} \sum_{j=2}^{k-2} \frac{1}{j}\right] \leq \\
\leq 2\left(\frac{1}{2}+\frac{1}{k-2}\right)^{s-2}\left[\frac{\pi^{2}}{3}+\frac{4}{k} \ln (k-2)\right] .
\end{gathered}
$$

Usando as estimativas (3.3) e (3.4) podemos estimar a expressão em (3.2), obtendo-se:

$$
\sum_{j=0}^{\infty} \omega_{k-j}^{-s} \omega_{j}^{-s} \leq \frac{1}{k^{s}}\left\{2+\frac{\pi^{2}}{6}+2\left(\frac{k}{k-1}\right)^{s}+\left(\frac{1}{2}+\frac{1}{k-2}\right)^{s-2}\left[\frac{\pi^{2}}{3}+\frac{4}{k} \ln (k-2)\right]\right\}
$$

Defina

$$
\sigma_{k}(s)=2+\frac{\pi^{2}}{6}+2\left(\frac{k}{k-1}\right)^{s}+\left(\frac{1}{2}+\frac{1}{k-2}\right)^{s-2}\left[\frac{\pi^{2}}{3}+\frac{4}{k} \ln (k-2)\right], k \geq 1 .
$$

Observe que $\frac{4}{k} \ln (k-2)$ é decrescente para $k \geq 6$. Logo, dado $M \geq 6$, temos

$$
\sigma_{k}(s) \leq \sigma_{M}(s) \forall k \geq M
$$

concluindo a demonstração do teorema.

Corolário 3.1.1. Dado $s \geq 2$, para cada natural $k \geq 0$ existe $\alpha_{k}(s)>0$ tal que

$$
\sum_{k_{1}+k_{2}=k} \frac{1}{\omega_{k_{1}}^{s}} \frac{1}{\omega_{k_{2}}^{s}} \leq \frac{\alpha_{k}(s)}{\omega_{k}^{s}}
$$

e, além disso, $\sup \left\{\alpha_{k}(s) ; k \in 0,1,2, \ldots\right\}=C(s)<\infty$.

Corolário 3.1.2. Dado $s \geq 2$, se $a \in \Omega^{s}, b \in \Omega^{s}$ então $\left(\sum_{k_{1}+k_{2}=k} a_{k_{1}} b_{k_{2}}\right)_{k=0}^{\infty} \in \Omega^{s}$. Mais especificamente, $\left\|\left(\sum_{k_{1}+k_{2}=k} a_{k_{1}} b_{k_{2}}\right)_{k=0}^{\infty}\right\|_{s} \leq C(s)\|a\|_{s}\|b\|_{s}$, onde $C(s)$ é como no teorema anterior.

Definição 3.1.2. Sejam $a=\left(a_{j}\right)_{j \geq 0}, \quad b=\left(b_{j}\right)_{j \geq 0} \in \Omega^{s}$, com $a_{j} \in \mathbb{C}$ e $b_{j} \in \mathbb{C}, j=$ $1,2,3, \ldots$ Definimos $(a * b)_{k}:=\sum_{k_{1}+k_{2}=k} a_{k_{1}} b_{k_{2}}$ e denominamos $a * b=\left((a * b)_{k}\right)_{k=0}^{\infty} o$ produto de convolução de a e b. 
Teorema 3.1.3. A função $f_{k}: \Omega^{s} \times \Omega^{s}: \rightarrow \mathbb{C}$ dada por

$$
f_{k}(a, b)=(a * b)_{k}
$$

é diferenciável e

$$
f_{k}^{\prime}(\bar{a}, \bar{b})(u, v)=\sum_{k_{1}+k_{2}=k} \bar{a}_{k_{1}} v_{k_{2}}+\bar{b}_{k_{2}} u_{k_{1}}
$$

Demonstração. Dados $(\bar{a}, \bar{b}),(u, v) \in \Omega^{s} \times \Omega^{s}$, a aplicação

$$
D(\bar{a}, \bar{b}):(u, v) \longmapsto \lim _{t \rightarrow 0} \frac{f_{k}(\bar{a}+t u, \bar{b}+t v)-f_{k}(\bar{a}, \bar{b})}{t}=\sum_{k_{1}+k_{2}=k} \bar{a}_{k_{1}} v_{k_{2}}+\bar{b}_{k_{2}} u_{k_{1}},
$$

é claramente linear em $(u, v)$. Além disso, para $h=\left(h_{1}, h_{2}\right) \in \Omega^{s} \times \Omega^{s}$, considerando a norma do máximo em $\Omega^{s} \times \Omega^{s}$ temos:

$$
\begin{gathered}
\frac{\left|f_{k}\left(\bar{a}+h_{1}, \bar{b}+h_{2}\right)-f_{k}(\bar{a}, \bar{b})-D(\bar{a}, \bar{b})\left(h_{1}, h_{2}\right)\right|}{\max \left\{\left\|h_{1}\right\|_{s},\left\|h_{2}\right\|_{s}\right\}} \leq \frac{\left|\sum_{k_{1}+k_{2}=k}\left(h_{1}\right)_{k_{1}}\left(h_{2}\right)_{k_{2}}\right|}{\left\|h_{1}\right\|_{s}}= \\
=\frac{\left|\left(h_{1} * h_{2}\right)_{k}\right|}{\left\|h_{1}\right\|_{s}} \leq \frac{\left\|\left(h_{1} * h_{2}\right)\right\|_{s}}{k^{s}\left\|h_{1}\right\|_{s}} \leq \frac{C(s)\left\|h_{1}\right\|_{s}\left\|h_{2}\right\|_{s}}{\left\|h_{1}\right\|_{s}}=C(s)\left\|h_{2}\right\|_{s} .
\end{gathered}
$$

Logo, fazendo $h \rightarrow 0$ na expressão acima, concluímos que $f_{k}^{\prime}(\bar{a}, \bar{b})(u, v)=D(\bar{a}, \bar{b})(u, v)$. Ou seja,

$$
f_{k}^{\prime}(\bar{a}, \bar{b})(u, v)=\sum_{k_{1}+k_{2}=k} \bar{a}_{k_{1}} v_{k_{2}}+\bar{b}_{k_{2}} u_{k_{1}}
$$

como queríamos provar.

Observação 3.1.1. Note que a aplicação linear $f_{k}^{\prime}(\bar{a}, \bar{b})$ é limitada. De fato, se $(u, v) \in$ $B_{\Omega^{s} \times \Omega^{s}}(0,1)$, ou seja, se $\|u\|_{s}<1$ e $\|v\|_{s}<1$, então,

$\left|f_{k}^{\prime}(\bar{a}, \bar{b})(u, v)\right|=\left|\sum_{k_{1}+k_{2}=k} \bar{a}_{k_{1}} v_{k_{2}}+\bar{b}_{k_{2}} u_{k_{1}}\right| \leq|\bar{a} * v|+|\bar{b} * u| \leq\|\bar{a}\|_{s}+\|\bar{b}\|_{s}, \forall(u, v) \in B_{\Omega^{s} \times \Omega^{s}}(0,1)$.

$\operatorname{Logo}$,

$$
\left\|f_{k}^{\prime}(\bar{a}, \bar{b})\right\| \leq\|\bar{a}\|_{s}+\|\bar{b}\|_{s}
$$

\subsection{Representação de Funções por Séries de Fourier}

Definição 3.2.1. Seja $f: \mathbb{R} \rightarrow \mathbb{C}$ uma função contínua p-periódica. Definimos a série de Fourier de $f$ por

$$
\sum_{k \in \mathbb{Z}} c_{k} e^{-i k l t}
$$

onde $l=\frac{2 \pi}{p}$ e $c_{k}=\frac{1}{p} \int_{0}^{p} \gamma(t) e^{-i k l t} \in \mathbb{C}$.

Note que, se $\gamma$ é uma função real, então $c_{-k}=\overline{c_{k}}$. De fato,

$$
c_{-k}=\frac{1}{p} \int_{0}^{p} \gamma(t) e^{i k l t}=\frac{1}{p} \int_{0}^{p} \overline{\gamma(t) e^{-i k l t}}=\overline{\left(\frac{1}{p} \int_{0}^{p} \gamma(t) e^{-i k l t}\right)}=\overline{c_{k}}, \quad k \in \mathbb{Z} .
$$

Em particular, $c_{0} \in \mathbb{R}$. 
Teorema 3.2.1. (Representação de Funções Periódicas Por Séries de Fourier) Suponha que $f \in C^{\infty}(\mathbb{R}, \mathbb{R})$ e que $f$ seja p-periódica. Então a série de Fourier de $f$ converge uniformemente para $f$ em $\mathbb{R}$. Além disso, a série de Fourier de $f^{\prime}$ pode ser obtida derivando a série de Fourier de $f$ termo a termo. Ou seja,

$$
f^{\prime}(t)=\sum_{k \in \mathbb{Z}}(-i k l) c_{k} e^{-i k l t} .
$$

Aplicando indutivamente o teorema acima às derivadas de ordem superior, obtemos

$$
f^{(j)}(t)=\sum_{k \in \mathbb{Z}}(-i k l)^{j} c_{k} e^{-i k l t} .
$$

Sendo a convergência uniforme em $\mathbb{R}$.

Teorema 3.2.2. (Decaimento dos coeficientes de Fourier) Sejam $\gamma \in C^{\infty}\left(\mathbb{R}, \mathbb{R}^{n}\right)$ periódica, $\gamma(t)=\sum_{k \in \mathbb{Z}} c_{k} e^{-i k l t}$ a representação de $\gamma(t)$ em série de Fourier, com $c_{k}=\left(c_{k, 1}, \ldots, c_{k, n}\right)^{T}$, e $s \geq 2$. Então, existe $\mu=\mu(s)>0$ tal que

$$
\left|c_{k, j}\right| \leq \frac{\mu(s)}{\omega_{k}^{s}}, \forall k \in \mathbb{Z}, j \in\{1, \ldots, n\}
$$

Demonstração. [4]

Note que o teorema acima nada afirma sobre decaimento dos coeficientes de Fourier de funções não periódicas.

Corolário 3.2.1. Se $\gamma \in C^{\infty}\left(\mathbb{R}, \mathbb{R}^{n}\right)$ é periódica e possui série de Fourier dada por $\gamma(t)=\sum_{k \in \mathbb{Z}} c_{k} e^{-i k l t}$, então $c=\left(c_{k}\right)_{k=0}^{\infty} \in \Omega^{s}$ qualquer que seja $s \geq 2$.

Observe que se $\gamma(t)$ é solução periódica de (3.1) então $\gamma \in C^{\infty}(\mathbb{R}, \mathbb{R})$. De fato, $\gamma^{\prime \prime \prime}(t)=$ $-\gamma^{\prime}(t)-\frac{\gamma^{2}}{2}+\lambda^{2} \Rightarrow \gamma^{\prime \prime \prime} \in C^{1}(\mathbb{R}, \mathbb{R}) \Rightarrow \gamma \in C^{4}(\mathbb{R}, \mathbb{R})$. Derivando, obtemos $\gamma^{(4)} \in C^{1}(\mathbb{R}, \mathbb{R})$, ou seja, $\gamma \in C^{5}(\mathbb{R}, \mathbb{R})$. Indutivamente, concluímos que $\gamma \in C^{k}(\mathbb{R}, \mathbb{R}), \forall k \in \mathbb{N}$, ou seja, $\gamma \in C^{\infty}(\mathbb{R}, \mathbb{R})$. Logo, pelo Teorema 3.2.1 e pelo Corolário 3.2.1 temos

Teorema 3.2.3. Se $\gamma(t)$ é solução p-periódica de (3.1) então ela pode ser expressa em termos de sua série de Fourier $\gamma(t)=\sum_{k \in \mathbb{Z}} c_{k} e^{-i k l t}$, onde $l=\frac{2 \pi}{p}$ e $c=\left(c_{k}\right)_{k=0}^{\infty} \in \Omega^{s}$, qualquer que seja $s \geq 2$.

Teorema 3.2.4. (Produto de séries de Fourier) Sejam $\alpha(t)=\sum_{k \in \mathbb{Z}} a_{k} e^{-i k l t}$ e $\beta(t)=$ $\sum_{k \in \mathbb{Z}} b_{k} e^{-i k l t}$ funções reais infinitamente diferenciáveis $\frac{2 \pi}{l}$-periódicas. Então,

$$
\alpha(t) \beta(t)=\sum_{k \in \mathbb{Z}}\left[\sum_{k_{1}+k_{2}=k} a_{k_{1}} b_{k_{2}}\right] e^{-i k l t} .
$$

Assim, com a notação da sessão anterior podemos escrever

$$
\alpha(t) \beta(t)=\sum_{k \in \mathbb{Z}}(a * b)_{k} e^{-i k l t},
$$

onde $a=\left(a_{k}\right)_{k=0}^{\infty}$ e $b=\left(b_{k}\right)_{k=0}^{\infty}$. 


\subsection{Reformulação do Problema}

Suponha que (3.1) possua uma órbita $p$-periódica $\gamma(t), t \in \mathbb{R}$. Pelo Teorema 3.2.3 podemos escrever

$$
\gamma(t)=\sum_{k \in \mathbb{Z}} c_{k} e^{-i k l t}, t \in \mathbb{R} .
$$

Tendo em vista os teoremas (3.2.1) e (3.2.4) e, substituindo (3.7) em (3.1), obtemos:

$$
\begin{gathered}
\gamma^{\prime \prime \prime}(t)+\gamma^{\prime}(t)+\frac{\gamma^{2}(t)}{2}=\lambda^{2} \Leftrightarrow \\
\Leftrightarrow \sum_{k \in \mathbb{Z}}\left[\left(\frac{1}{2} \sum_{k_{1}+k_{2}=k} c_{k 1} c_{k 2}\right)+i k l c_{k}\left(k^{2} l^{2}-1\right)-\delta_{0, k} \lambda^{2}\right] e^{-i k l t}=0 \\
\Leftrightarrow\left(\frac{1}{2} \sum_{k_{1}+k_{2}=k} c_{k 1} c_{k 2}\right)+i k l c_{k}\left(k^{2} l^{2}-1\right)-\delta_{0, k} \lambda^{2}=0, \quad \forall k \in \mathbb{Z} .
\end{gathered}
$$

Onde a última equivalência deve-se à unicidade dos coeficientes de Fourier.

Logo, definindo

$$
\begin{gathered}
F_{k}: \Omega^{s} \rightarrow \mathbb{C}, \\
F_{k}(c)=\frac{1}{2} \sum_{k_{1}+k_{2}=k}\left(c_{k_{1}} c_{k_{2}}\right)+i k l c_{k}\left(k^{2} l^{2}-1\right)-\delta_{0, k} \lambda^{2}, k \in \mathbb{Z},
\end{gathered}
$$

onde $c=\left(l,\left(c_{k}\right)_{k \geq 0}\right)$, com $c_{-k}=\bar{c}_{k}$ para $k>0$, decorre que o problema de encontrar uma órbita periódica para (3.1) torna-se equivalente a resolver as equações $F_{k}(x)=0, k \in \mathbb{Z}$ onde $x \in \Omega^{s}$ e $x_{-k}:=\bar{x}_{k}$.

Porém, se $F_{k}(x)=0$ para $k \geq 0$, teremos automaticamente que $F_{-k}(x)=0$. De fato, podemos escrever:

$$
\begin{aligned}
& F_{-k}(x)=\left(\frac{1}{2} \sum_{k_{1}+k_{2}=-k} x_{k_{1}} x_{k_{2}}\right)+i(-k) l x_{-k}\left((-k)^{2} l^{2}-1\right)-\delta_{0,-k} \lambda^{2}= \\
&=\left(\frac{1}{2} \sum_{k_{1}+k_{2}=k} x_{-k_{1}} x_{-k_{2}}\right)-i k l x_{-k}\left(k^{2} l^{2}-1\right)-\delta_{0, k} \lambda^{2}= \\
&=\left(\frac{1}{2} \sum_{k_{1}+k_{2}=k} \overline{x_{k_{1}} x_{k_{2}}}\right)+\overline{i k l x_{k}}\left(k^{2} l^{2}-1\right)-\delta_{0, k} \lambda^{2}= \\
&= \overline{\left(\frac{1}{2} \sum_{k_{1}+k_{2}=k} x_{k_{1}} x_{k_{2}}\right)+i k l x_{k}\left(k^{2} l^{2}-1\right)-\delta_{0, k} \lambda^{2}}= \\
&=\overline{F_{k}(x)} .
\end{aligned}
$$

Ou seja, $F_{-k}(x)=0 \Leftrightarrow \overline{F_{k}(x)}=0 \Leftrightarrow F_{k}(x)=0, \forall k \geq 0$. Assim, resolver o sistema de equações $F_{k}(x)=0, k \in \mathbb{Z}$, equivale a resolver as equações com índices não negativos, ou seja, a resolver o sistema

$$
F_{k}(x)=0, k \in\{0,1,2,3, \ldots\} .
$$

Usando a notação $x=\left(x_{k}\right)_{k=0}^{\infty} \operatorname{com} x_{0}=a_{0}+i l \in \mathbb{C}$ e $x_{k}=a_{k}+i b_{k} \in \mathbb{C}, k \geq 1$, abrindo as expressões de $F_{k}(x)$ em suas partes real e imáginária, obtemos:

$$
F_{0}(x)=\frac{1}{2} \sum_{k_{1}+k_{2}=0}\left(a_{k_{1}} a_{k_{2}}-b_{k_{1}} b_{k_{2}}\right)-\lambda^{2} \in \mathbb{R},
$$




$$
F_{k}(x)=\left(k l-k^{3} l^{3}\right) b_{k}+\frac{1}{2} \sum_{k_{1}+k_{2}=k}\left(a_{k_{1}} a_{k_{2}}-b_{k_{1}} b_{k_{2}}\right)+i\left[\left(k^{3} l^{3}-k l\right) a_{k}+\sum_{k_{1}+k_{2}=k} a_{k_{1}} b_{k_{2}}\right] .
$$

Denote por $F_{k, 1}(x)$ e $F_{k, 2}(x)$ as partes real e imaginária, respectivamente, de $F_{k}(x)$, para $k \geq 1$. Se $x \in \Omega^{s}$, tendo em vista as expressões de $F_{k, 1}(x)$ e $F_{k, 2}(x)$ dadas em (3.11) e (3.12) e o Corolário 3.1.1, segue que

$$
F(x):=\left(F_{0}(x), F_{1,1}(x), F_{1,2}(x), F_{2,1}(x), \ldots\right) \in \Omega^{s-3} .
$$

Observação 3.3.1. Note que decorre diretamente da Observação 3.1 .1 e das expressões de $F_{k, 1}$ e $F_{k, 2}$ dadas em (3.11) e (3.12) que as funções componentes de $F$ são diferenciáveis para todo $k \geq 0$ e suas derivadas num ponto qualquer de $\Omega^{s}$ são aplicações lineares limitadas.

Com o que foi discutido acima, fica demonstrada a primeira parte do seguinte teorema.

Teorema 3.3.1. Seja F como definida em (3.13), com $s>2$. A equação (3.1) possui solução periódica dada por $\gamma(t) \sum_{k \in \mathbb{Z}} c_{k} e^{-i k l t}$ se, e somente se, $F(c)=0$ onde $c=$ $\left(l, c_{0}, c_{1}, c_{2}, \ldots\right) \in \Omega^{s}$ com $l, c_{0} \in \mathbb{R} e c_{k} \in \mathbb{C}$.

Demonstração. $(\Rightarrow)$ Já foi provada na discussão acima.

$(\Leftarrow)$ Começamos provando que a série $\sum_{k \in \mathbb{Z}} c_{k} e^{-i k l t}$ converge uniformemente em $\mathbb{R}$.

De fato, como $c_{k} \in \Omega^{s}$, segue que $\sum_{k \in \mathbb{Z}}\left|c_{k}\right| \cdot\left|e^{-i k l t}\right|=\sum_{k \in \mathbb{Z}}\left|c_{k}\right| \leq \sum_{k \in \mathbb{Z}} \frac{\|c\|_{s}}{\omega_{k}^{s}}<\infty, \forall t \in \mathbb{R}$, pois $s>2$. Logo, pelo critério de Wierstrass, a série $\sum_{k \in \mathbb{Z}} c_{k} e^{-i k l t}$ converge uniformemente em $\mathbb{R}$ para alguma função

$$
\gamma(t)=\sum_{k \in \mathbb{Z}} c_{k} e^{-i k l t}
$$

Por outro lado, derivando a série de $\gamma(t)$ termo a termo obtemos a série $\sum_{k \in \mathbb{Z}}(-i k l) c_{k} e^{-i k l t}$. Como $\left(c_{k}\right)_{k \geq 0} \in \Omega^{s}$, segue que $\sum_{k \in \mathbb{Z}}\left|-i k l c_{k} e^{-i k l t}\right| \leq \sum_{k \in \mathbb{Z}}|k l| \frac{\|c\|_{s}}{\omega_{k}^{s}}=\sum_{k \in \mathbb{Z}}|l| \frac{\|c\|_{s}}{\omega_{k}^{s-1}}<\infty$, pois $s>2 \Rightarrow s-1>1$. Logo, a série $\sum_{k \in \mathbb{Z}}(-i k l) c_{k} e^{-i k l t}$ converge uniformemente em $\mathbb{R}$, donde podemos concluir que

$$
\gamma^{\prime}(t)=\sum_{k \in \mathbb{Z}}(-i k l) c_{k} e^{-i k l t}, t \in \mathbb{R}
$$

Por fim, como $c$ é raiz de $F$, então, por definição de $F_{k}$, temos

$$
\begin{gathered}
\left(\frac{1}{2} \sum_{k_{1}+k_{2}=k} c_{k 1} c_{k 2}\right)+i k l c_{k}\left(k^{2} l^{2}-1\right)-\delta_{0, k} \lambda^{2}=0, \quad \forall k \in \mathbb{Z} \Rightarrow \\
\Rightarrow(-i k l)^{3} c_{k}=i k^{3} l^{3} c_{k}=-\left(\frac{1}{2} \sum_{k_{1}+k_{2}=k} c_{k 1} c_{k 2}\right)+i k l c_{k}+\delta_{0, k} \lambda^{2} \forall k \in \mathbb{Z} .
\end{gathered}
$$

Como $\left(c_{k}\right)_{k \geq 0} \in \Omega^{s}$, concluímos que $\left(\frac{1}{2} \sum_{k_{1}+k_{2}=k} c_{k 1} c_{k 2}\right)_{k \geq 0} \in \Omega^{s}$ e $\left(i k l c_{k}\right)_{k \geq 0} \in \Omega^{s-1}$, ou seja, $\left\{(-i k l)^{3} c_{k}\right\}_{k \geq 0} \in \Omega^{s-1}$. Consequentemente, existe constante $B>0$ tal que $\left.\mid(-i k l)^{3} c_{k}\right) \mid \leq \frac{B}{k^{s-1}} \forall k \geq 1$. Donde segue que $\sum_{k \in \mathbb{Z}}\left|(-i k l)^{3} c_{k}\right| \leq \sum_{k \in \mathbb{Z}} \frac{B}{\omega_{k}^{s-1}}<\infty$, pois 
$s-1>1$, e, pelo critério de Wierstrass, a série $\sum_{k \in \mathbb{Z}}(-i k l)^{3} c_{k} e^{-i k l t}$ converge uniformemente em $\mathbb{R}$. Com isso, podemos concluir que

$$
\gamma^{\prime \prime \prime}(t)=\sum_{k \in \mathbb{Z}}(-i k l)^{3} c_{k} e^{-i k l t}, t \in R
$$

Por (3.14), (3.15) e (3.16) podemos escrever

$$
\begin{gathered}
\gamma^{\prime \prime \prime}(t)+\gamma^{\prime}(t)+\frac{\gamma(t)}{2}-\lambda^{2}= \\
=\sum_{k \in \mathbb{Z}}\left[(-i k l)^{3} c_{k}+(-i k l) c_{k}+\left(\frac{1}{2} \sum_{k_{1}+k_{2}=k} c_{k 1} c_{k 2}\right)-\delta_{0, k} \lambda^{2}\right] e^{-i k l t}= \\
=\sum_{k \in \mathbb{Z}} F_{k}(c) e^{-i k l t}=\sum_{k \in \mathbb{Z}} 0 e^{-i k l t}=0, \forall t \in \mathbb{R} .
\end{gathered}
$$

Ou seja, $\gamma(t)=\sum_{k \in \mathbb{Z}} c_{k} e^{-i k l t}$ é uma solução periódica de (3.1), como queríamos provar.

Observação 3.3.2. Note que, pelo Teorema 3.2.3, se $c \in \Omega^{\text {s }}$ satistaz o teorema acima para algum $s>2$, então $c \in \Omega^{\tilde{s}}$ qualquer que seja $\tilde{s}>0$

A fim de podermos aplicar o Teorema 3.3.1, no que segue, tomaremos sempre $s>2$.

Note que, se estivermos considerando $s>2$, o Teorema 3.3.1 estabelece uma correspondência entre raízes de $F: \Omega^{s} \rightarrow \Omega^{s-3}$ e as órbitas periódicas de (3.1). A cada raiz corresponde uma órbita periódica e a cada órbita podem corresponder várias raízes. E, como veremos, tais raízes não são isoladas. Destacamos essa correspondência entre raízes e órbitas periódicas na seguinte definição.

Definição 3.3.1. Sejam F como dada em (3.13), com $s>2$, $\Gamma$ uma órbita periódica de (3.1) e $\gamma(t)=\sum_{k \in \mathbb{Z}} c_{k} e^{-i k l t}$ uma solução periódica de (3.1) que parametriza essa órbita, ou seja, $\Gamma=\{\gamma(t) ; t \in \mathbb{R}\}$. Pelo Teorema 3.3 .1 , sabemos que $c=\left(l,\left(c_{k}\right)_{k \geq 0}\right) \in \Omega^{s}$ é uma raiz de $F$. Desse modo, diremos que $c=\left(l,\left(c_{k}\right)_{k \geq 0}\right) \in \Omega^{s}$ é uma raiz de $F$ associada ̀̀ órbita $\Gamma$.

Note que, para cada solução que parametriza a órbita $\Gamma$, teremos uma raiz de $F$ associada a $\Gamma$. Ou seja, podem haver várias raízes de $F$ associadas a uma mesma órbita. Basta que existam várias parametrizações por séries de Fourier desta mesma órbita. Veremos, na seção seguinte, que este é realmente o caso.

\subsection{Isolando as raízes}

Teorema 3.4.1. Sejam $f \in C^{1}\left(\mathbb{R}^{n}, \mathbb{R}^{n}\right)$ e $\Gamma$ uma órbita periódica do sistema autônomo $x^{\prime}=f(x)$. Suponha que $\Gamma$ seja parametrizada por uma solução periódica $\gamma(t)$ desse sistema. Nesse caso, uma função $\lambda(t)$ é solução do sistema $x^{\prime}=f(x)$ e possui órbita $\Gamma$ se, e somente se, $\lambda(t)=\gamma_{\tau}(t):=\gamma(t+\tau)$, para algum $\tau \in \mathbb{R}$.

Demonstração. Seja $\lambda(t)$ uma solução do sistema $x^{\prime}=f(x)$ cuja órbita é dada por $\Gamma$. Provemos que $\lambda$ é da forma $\gamma_{\tau}(t)$ para algum $\tau \in \mathbb{R}$. 
De fato, seja $x:\left(t, x_{0}\right) \longmapsto x\left(t, x_{0}\right)$ o fluxo gerado pelo campo vetorial $f$. Como $\lambda(t)$ e $\gamma(t)$ parametrizam a mesma curva $\Gamma$, deve existir $\tau \in \mathbb{R}$ tal que $\gamma(\tau)=\lambda(0) \in \Gamma$. Ainda, pela propriedade básica dos fluxos, segue que

$$
\lambda(t)=x(t, \lambda(0))=x(t, \gamma(\tau))=x(t, x(\tau, \gamma(0)))=x(t+\tau, \gamma(0))=\gamma(t+\tau)=\gamma_{\tau}(t) .
$$

Reciprocamente, note que

$$
\gamma_{\tau}^{\prime}(t)=(\gamma(t+\tau))^{\prime}=\gamma^{\prime}(t+\tau)=f(\gamma(t+\tau))
$$

ou seja, $\gamma_{\tau}$ é solução do sistema autônomo $x^{\prime}=f(x)$, qualquer que seja $\tau \in \mathbb{R}$. Resta provar que as funções $\gamma_{\tau}(t)$ parametrizam a mesma órbita que $\gamma(t)$. De fato, seja $\Gamma$ a órbita da solução $\gamma(t)$. Assim, dado $\gamma\left(t_{0}\right) \in \Gamma$, temos que

$$
\gamma\left(t_{0}\right)=\gamma\left(t_{0}-\tau+\tau\right)=\gamma_{\tau}\left(t_{0}-\tau\right)
$$

Ou seja, $\Gamma$ está contida na órbita de $\gamma_{\tau}(t)$. Ainda, dado $t_{0} \in \mathbb{R}$, temos

$$
\gamma_{\tau}\left(t_{0}\right)=\gamma\left(t_{0}+\tau\right) \in \Gamma
$$

Ou seja, a órbita gerada por $\gamma_{\tau}(t)$ está contida em $\Gamma$. Logo, as soluções $\gamma_{\tau}(t)$ e $\gamma(t)$ parametrizam a mesma órbita $\Gamma$, qualquer que seja $\tau \in \mathbb{R}$.

Corolário 3.4.1. Considere $F$ como dada em (3.13) com $s>2$. Sejam $\Gamma$ uma órbita periódica de (3.1) e $\gamma(t)=\sum_{k \in \mathbb{Z}} c_{k} e^{-i k l t}$ uma solução periódica de (3.1) que parametriza a curva $\Gamma$. Nesse caso, as únicas raízes de $F$ associadas à órbita $\Gamma$ são da forma:

$$
\left(l, c_{0}, c_{1}^{\tau}, c_{2}^{\tau}, \ldots\right), \text { onde } c_{k}^{\tau}=c_{k} e^{-i k l \tau}, k \geq 1, \tau \in \mathbb{R} .
$$

Demonstração. As raízes de $F$ associadas a uma órbita $\Gamma$ ficam determinadas pelos coeficientes de Fourier das soluções que parametrizam essa órbita. Mas o sistema de EDO's equivalente à equação (3.1) é autônomo. Logo, segue do Teorema 3.4.1 que as soluções periódicas que parametrizam a órbita $\Gamma$ são dadas por $\gamma_{\tau}(t)=\gamma(t+\tau)$, cujas séries de Fourier são dadas por

$$
\gamma_{\tau}(t)=\gamma(t+\tau)=\sum_{k \in \mathbb{Z}} c_{k} e^{-i k l(t+\tau)}=\sum_{k \in \mathbb{Z}}\left(c_{k} e^{-i k l \tau}\right) e^{-i k l t}=\sum_{k \in \mathbb{Z}} c_{k}^{\tau} e^{-i k l t}, c_{k}^{\tau}:=c_{k} e^{-i k l \tau} .
$$

Logo, as únicas raízes de $F$ associadas à órbita $\Gamma$ são da forma $\left(l, c^{\tau}\right)$ onde $c^{\tau}=\left(c_{k} e^{-i k l \tau}\right)_{k \geq 0}$.

Teorema 3.4.2. Fixado $s>2$, a função $F: \Omega^{s} \rightarrow \Omega^{s-3}$ dada por (3.13) não possui raízes isoladas.

Demonstração. Mostremos que uma raiz qualquer $c=\left(l, c_{0}, c_{1}, c_{2}, \ldots\right)$ da função $F$ dada em (3.13) não é isolada. Seja $c^{\tau}=\left\{c_{k} e^{-i k l \tau}\right\}_{k=0}^{\infty}$. Pelo corolário anterior, $d:=\left(l, c^{\tau}\right)$ é raiz de $F$, qualquer que seja $\tau \in \mathbb{R}$. Pela Observação 3.3.2, temos que $c, d \in \Omega^{\tilde{s}}, \forall \tilde{s}>s$. Em particular, $c, d \in \Omega^{s+2}$. Assim, por definição de $\|\cdot\|_{s+2}$, podemos escrever

$$
\left|c_{k}\right| \leq \frac{\|c\|_{s+2}}{k^{s+2}}, \forall k \geq 1 \Rightarrow\left|c_{k}\right| k^{s} \leq \frac{\|c\|_{s+2}}{k^{2}}, \forall k \geq 1
$$


Portanto, dado $N>0$, temos

$$
\sup _{k \geq N}\left|c_{k}\right| k^{s} \leq \sup _{k \geq N} \frac{\|c\|_{s+2}}{k^{2}}=\frac{\|c\|_{s+2}}{N^{2}}
$$

Analogamente, concluímos que

$$
\sup _{k \geq N}\left|c_{k}^{\tau}\right| k^{s} \leq \frac{\left\|c^{\tau}\right\|_{s+2}}{N^{2}}=\frac{\|c\|_{s+2}}{N^{2}}, \forall \tau \in \mathbb{R}
$$

Com isso, podemos estimar a distância entre as soluções $c$ e $d$ no espaço $\Omega^{s}$. De fato, temos:

$$
\begin{gathered}
\|c-d\|_{s}=\max \left\{\max _{0 \leq k \leq N-1}\left|c_{k}-c_{k} e^{-i k l \tau}\right| \cdot \omega_{k}^{s}, \sup _{k \geq N}\left|c_{k}-c_{k}^{\tau}\right| \cdot \omega_{k}^{s}\right\} \leq \\
\leq \max \left\{\max _{0 \leq k \leq N-1}\left|c_{k}\right| \cdot \omega_{k}^{s} \cdot\left|1-e^{-i k l \tau}\right|, \sup _{k \geq N}\left|c_{k}\right| \cdot \omega_{k}^{s}+\sup _{k \geq N}\left|c_{k}^{\tau}\right| \cdot \omega_{k}^{s}\right\} \leq \\
\leq \max \left\{\|c\|_{s} \max _{0 \leq k \leq N-1}\left|1-e^{-i k l \tau}\right|, \frac{\|c\|_{s+2}}{N^{2}}+\frac{\|c\|_{s+2}}{N^{2}}\right\} .
\end{gathered}
$$

Agora, dado $\epsilon>0$, tome $N$ tal que

$$
2 \frac{\|c\|_{s+2}}{N^{2}} \leq \epsilon
$$

Pela continuidade da função exponencial, para cada $0 \leq k \leq N-1$ podemos tomar $\tau_{k}<0$ com módulo suficientemente pequeno tal que $\left|1-e^{-i k l t}\right|<\frac{\epsilon}{\|c\|_{s}}, \forall t \in\left[\tau_{k}, 0\right]$. Logo, tomando $\tau=\min \left\{\tau_{0}, \ldots, \tau_{N-1}\right\}$, temos $\left|1-e^{-i k l \tau}\right|<\frac{\epsilon}{\|c\|_{s}}, \forall k \in\{0, \ldots, N-1\}$. Ou seja,

$$
\max _{0 \leq k \leq N-1}\left|1-e^{-i k l \tau}\right| \leq \frac{\epsilon}{\|c\|_{s}} .
$$

Podemos estimar (3.17) tendo em vista (3.18) em (3.19), obtendo

$$
\left\|c-c^{\tau}\right\|_{s} \leq \epsilon
$$

Assim, concluímos que para todo $\epsilon>0$ podemos encontrar uma raiz $c^{\tau}$ de $F$ na bola $B_{\Omega^{s}}(c, \epsilon)$ de centro $c$ e raio $\epsilon$ no espaço $\Omega^{s}$. Ou seja, $c$ não é uma raiz isolada de $F$. Como $c$ é uma raiz qualquer de $F$, segue que $F$ não possui raízes isoladas.

Porém, como vimos no preâmbulo desse capítulo, nossa estratégia para a demonstração de existência de raízes baseia-se na determinação, via teorema do ponto fixo de Banach, de pontos fixos isolados de um operador da forma $T(x)=x-A F(x)$. Na prática, queremos provar que $T$ é uma contração numa bola no espaço das raízes de $F$. Logo, se fossemos bem sucedidos em provar que $T$ é uma contração, garantindo a existência de um ponto fixo isolado de $T$, automaticamente estaríamos garantindo a existência de uma raiz isolada de $F$, o que, pelo Teorema 3.4.2, é absurdo.

No que segue, discutimos como acrescentar uma condição sobre as raízes de $F$ de modo que raízes associadas a uma mesma órbita periódica de (3.1) fiquem isoladas umas das outras. A essa condição chamaremos condição de fase.

Note que a condição de fase garante apenas que raízes associadas a uma mesma órbita fiquem isoladas entre si. O isolamento global das raízes de $F$ numa certa região do espaço das raízes depende do isolamento das órbitas periódicas numa região do espaço de fase. Em outras palavras, a ocorrência de órbitas não isoladas no espaço de fase com períodos também arbitráriamente próximos torna nossa estratégia imcompatível com a estrutura do problema. Portanto, a eficácia do método fica sujeita à existencia de órbitas periódicas isoladas no espaço de fase. 
Teorema 3.4.3. Suponha que (3.1) possua uma órbita periódica $\Gamma$. Nesse caso, existe $N \geq 1$ tal que o problema

$$
\left\{\begin{array}{l}
F\left(l, x_{0}, x_{1}, \ldots\right)=0 \\
R e\left\{x_{N}\right\}=0
\end{array}\right.
$$

possui finitas soluções.

Demonstração. Seja $\gamma(t)=\sum_{k \in \mathbb{Z}} c_{k} e^{-i k l t}$ uma solução periódica de (3.1) que parametriza a órbita $\Gamma$. Pelo Corolário 1.4.1, as únicas raízes de $F$ associadas à órbita $\Gamma$ são dadas por $c^{\tau}=\left(l, c_{0}, c_{1}^{\tau}, c_{1}^{\tau}, \ldots\right), \tau \in R$. Como $\gamma(t)$ não é constante, existe $c_{N} \neq 0$, para algum $N \geq 1$. Seja $c_{N}=\left|c_{N}\right| e^{i \theta}$. Por definição, temos $c_{N}^{\tau}:=c_{N} e^{-i N l \tau}$. Assim, podemos escrever:

$$
\begin{gathered}
\operatorname{Re}\left\{c_{N}^{\tau}\right\}=0 \Leftrightarrow \operatorname{Re}\left\{\left|c_{N}\right| e^{i \theta} c_{N} e^{-i N l \tau}\right\}=0 \Leftrightarrow \\
\Leftrightarrow \operatorname{Re}\left\{\left|c_{N}\right| e^{i(\theta-N l \tau)}\right\}=0 \Leftrightarrow \theta-N l \tau=k \frac{\pi}{2}, k \in\{ \pm 1, \pm 3, \pm 5, \ldots\} \Leftrightarrow \\
\Leftrightarrow \tau=\frac{\theta}{l N}-k \frac{\pi}{2 l N}, k \in\{ \pm 1, \pm 3, \pm 5, \ldots\} .
\end{gathered}
$$

Defina $\tau_{k}=\frac{\theta}{l N}-k \frac{\pi}{2 l N}, k \in\{ \pm 1, \pm 3, \pm 5, \ldots\}$. Assim, as únicas raízes de $F$ satisfazendo a condição de fase $\operatorname{Re}\left\{c_{N}^{\tau}\right\}=0$ são dadas por $c^{\tau_{k}}, k \in\{ \pm 1, \pm 3, \pm 5, \ldots\}$. Além disso, para todo $k \geq 1$, temos :

$$
c_{k}^{\tau_{n}}=c_{k} e^{-i k l \tau_{n}}=c_{k} e^{-i k l\left(\frac{\theta}{l N}-n \frac{\pi}{2 l N}\right)}=c_{k} e^{-i k \frac{\theta}{N}}\left(e^{i n \frac{\pi}{2 N}}\right)^{k}, n \in\{ \pm 1, \pm 3, \pm 5, \ldots\} .
$$

Por outro lado, note que

$$
\left\{e^{i n \frac{\pi}{2 N}} ; n= \pm 1, \pm 3, \pm 5, \ldots\right\}=\left\{e^{i n \frac{\pi}{2 N}} ; n=1,3, \ldots 4 N-1\right\}
$$

Logo,

$$
\left\{c^{\tau_{n}} ; n= \pm 1, \pm 3, \pm 5, \ldots\right\}=\left\{c^{\tau_{1}}, c^{\tau_{3}}, \ldots, c^{\tau_{4 N-1}}\right\}
$$

Assim, o conjunto solução do problema

$$
\left\{\begin{array}{l}
F\left(l, x_{0}, x_{1}, \ldots\right)=0 \\
R e\left\{x_{N}\right\}=0
\end{array}\right.
$$

é dado por

$$
S=\left\{c^{\tau_{1}}, c^{\tau_{3}}, \ldots, c^{\tau_{4 N-1}}\right\}
$$

Desse modo, a condição extra $\operatorname{Re}\left\{x_{N}\right\}=0$ "seleciona" um número finito de raízes $F$. Tecnicamente, acrescentar essa condição de fase equivale a acrescentar a função coordenada $x \mapsto R e\left\{x_{N}\right\}$, às funções coordenadas de $F$. Assim, para uniformização da notação, defina $F_{0,1}(x):=\operatorname{Re}\left\{x_{N}\right\}$ e $F_{0,2}(x):=F_{0}(x)$. Desse modo, podemos redefinir $F$ por

$$
\begin{gathered}
F: \Omega^{s} \rightarrow \Omega^{s-3} \\
F(x)=\left(F_{0,1}(x), F_{0,2}(x), F_{1,1}(x), F_{1,2}(x), F_{2,1}(x), F_{2,2}(x), \ldots\right),
\end{gathered}
$$

de modo que as raízes dessa nova $F$ associadas a uma mesma órbita $\Gamma$, pela discussão acima, estarão isoladas, validando a abordagem via terema do ponto fixo de Banach com a finalidade de buscar por raízes de $F$. De agora em diante passaremos a denotar $F_{0}(x)=F_{0,1}(x)+i F_{0,2}(x)$. 


\subsection{Redução a um Problema de Ponto fixo}

Nosso objetivo daqui em diante é provar existência de raízes de $F$ como definida em (3.20) para algum $s>2$. De acordo com o Teorema 3.3.1, isso é suficiente a provar a existência de uma órbita periódica de (3.1).

A seguir descrevemos nossa estratégia, inspirada no teorema do ponto fixo de Banach e no Método de Newton.

O primeiro passo é encontrar uma aproximação para uma raiz de $F$. Será numa vizinhança dessa aproximação que buscaremos por raízes exatas. Para encontrar aproximações de raízes de $F$, consideraremos a versão "truncada" do problema, ou seja, consideraremos um número finito de coeficientes de Fourier, gerando um numero finito de equações em finitas variáveis. Este problema pode ser resolvido pelo Método de Newton clássico, ou seja, em dimensões finitas.

Para provar a existência de raízes nas proximidades da aproximação encontrada no primeiro passo buscaremos por uma vizinhança desta aproximação na qual um determinado operador (inspirado no Método de Newton) será uma contração, cujos pontos fixos, garantidos pelo teorema do ponto fixo de Banach, serão raízes de $F$, demonstrando, de acordo com o Teorema 3.3.1, a existência de órbitas periódicas de (3.1).

\subsubsection{Truncamento}

Sabemos que os coeficientes de Fourier de uma solução periódica de (3.1) tendem rapidamente a zero. Ou seja, o comportamento dessas funções são dominados pelos primeiros coeficientes. Assim, escolhendo $m$ suficientemente grande, esperamos que, obtendo coeficientes $\bar{c}_{0}, \ldots \bar{c}_{m-1}$ e número real $l>0$ tais que

$$
F^{m}\left(\bar{l}, \bar{c}_{0}, \ldots \bar{c}_{m-1}\right):=\left(F_{0}\left(\bar{l}, \bar{c}_{0}, \ldots \bar{c}_{m-1}, 0,0, \ldots\right), \ldots, F_{m-1}\left(\bar{l}, \bar{c}_{0}, \ldots \bar{c}_{m-1}, 0,0, \ldots\right)\right) \approx 0
$$

então a função

$$
\gamma^{m}(t)=\sum_{|k| \leq m-1} \bar{c}_{k} e^{-i k l t}=\sum_{k \in \mathbb{Z}} \bar{c}_{k} e^{-i k l t}, c_{k}=0 \text { se }|k| \geq m
$$

aproxima-se de uma solução periódica de (3.1). Ou seja, $\left(\bar{l}, \bar{c}_{0}, \ldots \bar{c}_{m-1}, 0_{\infty}\right)$ aproxima-se de uma raiz de $F$. Em particular, se formos capazes de provar a existência de tais coeficientes satisfazendo exatamente $F^{m}\left(\bar{l}, \bar{c}_{0}, \ldots \bar{c}_{m-1}\right)=0$ então $\gamma^{m}(t)$ é exatamente uma solução periódica de (3.1).

Note que o Método de Newton nos permite obter raízes aproximadas $\bar{c}=\left(\bar{l}, \bar{c}_{0}, \ldots \bar{c}_{m-1}\right) \in$ $\mathbb{R}^{2 m}$ da função $F^{m}: \mathbb{R}^{2 m} \rightarrow \mathbb{R}^{2 m}$ definida acima. Além disso, quanto maior for $m$, mais próxima de uma solução periódica de (3.1) estará a função

$$
\gamma^{m}(t)=\sum_{|k| \leq m-1} \bar{c}_{k} e^{-i k l t}
$$

ou, equivalentemente, mais próxima de uma raiz de $F$ estará o elemento $(\bar{c}, 0,0, \ldots)=$ $\left(\bar{c}, 0_{\infty}\right)$.

Resumindo, na prática a primeira etapa do método consiste em:

1. Implementar a função $F^{m}(c)=\left(F_{0,1}^{m}(c), F_{0,2}^{m}(c), \ldots, F_{m-1,1}^{m}(c), F_{m-1,2}^{m}(c)\right), c \in \mathbb{R}^{2 m}$, onde $m$ é um parâmetro que se possa variar. 
2. Implementar a matriz Jacobiana da derivada $D F^{m}(c)$;

3. Implementar o Método de Newton para a função $F^{m}$, obtendo $\bar{c} \in \mathbb{R}^{2 m}$ tal que $F^{m}(\bar{c}) \approx 0$.

Na Tabela 3.1 mostramos o resultado obtido pela implementação desses passos para a equação de Michelson.

\subsubsection{Operador de Newton}

Vamos tentar provar a existência de uma raiz de $F$ numa vizinhança do ponto $\bar{c}=$ $\left(\bar{l}, \bar{c}_{0}, \ldots \bar{c}_{m-1}, 0_{\infty}\right)$, obtido na etapa anterior. (Aqui estamos fazendo um abuso de notação ao representar um vetor finito e o correspondente em $\Omega^{s}$ pelo símbolo $\bar{c}$. Faremos isso outras vezes ao longo desse trabalho.) Para isso, buscaremos por uma contração numa pequena bola $B\left[\left(\bar{c}, 0_{\infty}\right), r\right]$ de raio $r$ centrada em $\left(\bar{c}, 0_{\infty}\right)$ da forma

$$
T(x)=x-A \cdot F(x),
$$

com $A: \Omega^{s-3} \rightarrow \Omega^{s}$ inversível.

Exigimos a invertibilidade de $A$ a fim de que pontos fixos de $T$ correspondam a raízes de $F$. De fato, se $\bar{x}$ é um ponto fixo de $T$ e se $A$ é invertível, podemos escrever:

$$
T(\bar{x})=\bar{x} \Leftrightarrow \bar{x}-A F(\bar{x})=\bar{x} \Leftrightarrow A F(\bar{x})=0 \Rightarrow F(\bar{x})=A^{-1} \cdot 0=0 .
$$

Exigimos que $A$ tome valores em $\Omega^{s}$ para que $T$ seja de fato um operador em $\Omega^{s}$. Sem essa condição, o teorema do ponto fixo e Banach não seria aplicável ao operador $T$.

Se formos capazes de encontrar um operador $A$ e um raio $r>0$ de modo que o operador $T$ definido acima seja uma contração e que $T: B_{\Omega^{s}}[\bar{c}, r] \rightarrow B_{\Omega^{s}}[\bar{c}, r]$, o teorema do ponto fixo de Banach garantira a existência de um ponto fixo pelo operador $T$ e, portanto, de uma raiz de $F$, como queremos provar.

A princípio poderiamos escolher qualquer outro tipo de operador com pontos fixos correspondendo a raízes de $F$ e tentar transformálo numa contração nalguma região de $\Omega^{s}$, talvez existam escolhas até mais eficiente (menor constante de contração). Porém, nossa referência para esse trabalho é o Método de Newton em espaços Euclidianos, que faz uso desse tipo de operador.

Vale ressaltar que a vantagem de procurar por pontos fixos em vez de raízes é que temos em mãos o teorema de ponto fixo de Banach, que nos garante a existência de pontos fixos de contrações e ainda nos fornece um método iterativo extremamente simples para calculálos com grande precisão. Em contrapartida, não conhecemos fórmulas ou abordagens centradas no estudo da própria função $F$ para determinação de raízes.

Dito isso, passamos a buscar por um operador $A$ que aumente as possibilidades do operador de Newton $T(x)=x-A \cdot F(x)$ ser uma contração.

\subsubsection{Construção do operador $A$}

Na prática, precisamos estabelecer um operador $A$ adequado. Ou seja, que potencialize a possibilidade de $T$ ser uma contração e faça com que que $T: \Omega^{s} \rightarrow \Omega^{s}$. Analogamente ao Método de Newton em dimensões finitas, somos sugestionados a tomar o operador A como sendo a inversa da derivada de $F$ num ponto próximo a uma de suas raízes. No nosso caso, teríamos $A \approx\left[F^{\prime}\left(\bar{c}, 0_{\infty}\right)\right]^{-1}$. Mostremos que essa é, de fato, uma boa escolha. 
Sejam $\bar{x}=\left(\bar{c}, 0_{\infty}\right)$ e $B(\bar{x}, r) \subset \Omega^{s}$ a bola de centro $\bar{x}$ e raio $r$ segundo a norma $\|\cdot\|_{s}$. Assuma que A seja tal que $T: \Omega^{s} \rightarrow \Omega^{s}$. Assim, pela desigualdade do valor médio, temos

$$
\|T(x)-T(y)\|_{s} \leq\left(\sup _{\Theta \in B(\bar{x}, r)}\|D T(\Theta)\|\right)\|x-y\|_{s}, \forall x, y \in B(\bar{x}, r) .
$$

Portanto, a fim de tornar $T$ uma contração, devemos tomar $A$ de modo que sup $\|D T(\Theta)\|<$

1. Com essa finalidade, impomos que $\|D T(\Theta)\|=\|I d-A \circ D F(\Theta)\| \approx 0 \quad \forall \Theta \in(\bar{x}, r)$, ou, equivalentemente, $A \approx(D F(\Theta))^{-1} \forall \Theta \in(\bar{x}, r)$. Fazemos isso tomando

$$
A \approx(D F(\bar{x}))^{-1} \text {. }
$$

Assim, a descrição do operador $A$ depende de, primeiramente, conhecermos o operador linear $D F(\bar{x})$, e, em seguida, buscarmos uma aproximação para sua inversa, já que a inversa exata é de difícil obtenção.

\section{Representação Jacobiana da derivada $D F(x)$}

Fixado $s>2$ e dado $v=\left(v_{0,1}, v_{0,2}, v_{1,1}, v_{1,2}, v_{2,1}, v_{2,2}, \ldots\right) \in \Omega^{s}$, teremos $v=\sum_{k=0}^{\infty} v^{k} \operatorname{com}$ $v^{k}=\left(0, \ldots, 0, v_{k, 1}, v_{k, 2}, 0,0 \ldots\right)$. De fato, dado $\bar{s}<s$ temos que $v \in \Omega^{\bar{s}}$. Assim, podemos escrever:

$$
\begin{aligned}
\left\|v-\sum_{k=0}^{n} v^{k}\right\|_{\bar{s}}= & \left\|\left(0, \ldots, 0, v_{n+1,1}, v_{n+1,2}, v_{n+2,1}, v_{n+2,2}, \ldots\right)\right\|_{\bar{s}}= \\
& \left.=\sup _{j>n}\left\{\|\left(v_{j, 1}, v_{j, 2}\right)\right) \|_{\infty} \cdot j^{\bar{s}}\right\} .
\end{aligned}
$$

Como $v \in \Omega^{s}$, temos que $\left\|\left(v_{j, 1}, v_{j, 1}\right)\right\|_{\infty} \leq \frac{\|v\|_{s}}{j^{s}}$, para todo $j>0$. Logo,

$$
\left\|v-\sum_{k=0}^{n} v^{k}\right\|_{\bar{s}}=\sup _{j>n}\left\{\left\|\left(v_{j, 1}, v_{j, 2}\right)\right\|_{\infty} \cdot j^{\bar{s}}\right\} \leq \sup _{j>n}\left\{\frac{\|v\|_{s} \cdot j^{\bar{s}}}{j^{s}}\right\}<\frac{\|v\|_{s}}{n^{s-\bar{s}}} .
$$

Fazendo $n \rightarrow \infty$ em (3.22) concluímos que $v=\sum_{k=0}^{\infty} v^{k}$. Portanto, para cada $k \in$ $\{0,1,2, \ldots\}$, pela continuidade de $D F_{k}(\bar{x})$ em $\Omega^{s} \subset \Omega^{\bar{s}}$ com relação à norma $\|\cdot\|_{\bar{s}}$ temos

$$
\begin{gathered}
D F_{k}(\bar{x}) v=D F_{k}(\bar{x})\left(\lim _{n \rightarrow \infty} \sum_{j=0}^{n} v^{j}\right)=\lim _{n \rightarrow \infty} \sum_{j=0}^{n} D F_{k}(\bar{x}) v^{j}=\sum_{j=0}^{\infty} D F_{k}(\bar{x})\left(0, \ldots, 0, v_{j, 1}, v_{j, 2}, 0,0, \ldots\right)= \\
=\sum_{j=0}^{\infty} D F_{k}(\bar{x})\left(0, \ldots, 0, v_{j, 1}, 0,0 \ldots\right)+D F_{k}(\bar{x})\left(0, \ldots, 0, v_{j, 2}, 0,0, \ldots\right)= \\
=\sum_{j=0}^{\infty} \frac{\partial F_{k}}{\partial a_{j}}(\bar{x}) v_{j, 1}+\frac{\partial F_{k}}{\partial b_{j}}(\bar{x}) v_{j, 2}= \\
=\sum_{j=0}^{\infty} \frac{\partial F_{k}}{\partial x_{j}}(\bar{x}) v_{j}
\end{gathered}
$$

onde

$$
\frac{\partial F_{k}}{\partial x_{j}}=\left(\begin{array}{ll}
\frac{\partial F_{k, 1}}{\partial a_{j}} & \frac{\partial F_{k, 1}}{\partial b_{j}} \\
\frac{\partial F_{k, 2}}{\partial a_{j}} & \frac{\partial F_{k, 2}}{\partial b_{j}}
\end{array}\right)
$$


e

$$
v_{j}=\left(\begin{array}{c}
v_{j, 1} \\
v_{j, 2}
\end{array}\right)
$$

Como,

$$
D F(\bar{x}) v=\left(D F_{0}(\bar{x}) v, D F_{1}(\bar{x}) v, \ldots\right)=\left(\sum_{j=0}^{\infty} \frac{\partial F_{0}}{\partial x_{j}}(\bar{x}) v_{j}, \sum_{j=0}^{\infty} \frac{\partial F_{1}}{\partial x_{j}}(\bar{x}) v_{j}, \ldots\right),
$$

segue que $D F(\bar{x}) v$ pode ser expresso na forma matricial abaixo:

$$
D F(\bar{x}) v=\left(\begin{array}{lll}
\frac{\partial F_{0}}{\partial x_{0}}(\bar{x}) & \frac{\partial F_{0}}{\partial x_{1}}(\bar{x}) & \cdots \\
\frac{\partial F_{1}}{\partial x_{0}}(\bar{x}) & \frac{\partial F_{1}}{\partial x_{1}}(\bar{x}) & \cdots \\
\vdots & \vdots & \ddots
\end{array}\right)\left(\begin{array}{l}
v_{0} \\
v_{1} \\
v_{2} \\
v_{3} \\
\vdots
\end{array}\right)
$$

\section{Inversa aproximada da derivada $D F(x)$}

Queremos encontrar uma aproximação para $D F(x)$ que seja simples o suficiente para que possamos conhecer sua inversa. A seguinte análise nos permite obter tal aproximação.

Sejam dados $s>2, m>0, \bar{c}=\left(\bar{l}, \bar{a}_{0}, \bar{a}_{1}, \bar{b}_{1}, \ldots, \bar{a}_{m-1}, \bar{b}_{m-1}\right) \in \mathbb{R}^{2 m}$ e $\bar{x}=\left(\bar{c}, 0_{\infty}\right) \in \Omega^{s}$. A fim de obtermos uma aproximação para o operador $D F(\bar{x})$ dado pela matriz em (3.25), precisamos explicitar as derivadas parciais presentes em suas componentes. Calculando diretamente obtemos:

$$
\begin{gathered}
\frac{\partial F_{k}}{\partial x_{j}}(\bar{x})=\delta_{k, j}\left(k \bar{l}-k^{3} \bar{l}^{3}\right)\left(\begin{array}{cc}
0 & 1 \\
-1 & 0
\end{array}\right)+\left(\begin{array}{cc}
\bar{a}_{k-j}+\bar{a}_{k+j} & \bar{b}_{k+j}-\bar{b}_{k-j} \\
\bar{b}_{k-j}+\bar{b}_{k+j} & \bar{a}_{k-j}-\bar{a}_{k+j}
\end{array}\right) ; k \geq 1, j \geq 1 . \\
\frac{\partial F_{k}}{\partial x_{0}}(\bar{x})=\left(\begin{array}{cc}
\left(k-3 k^{3} \bar{l}^{2}\right) \bar{b}_{k} & \bar{a}_{k} \\
\left(3 k^{3} \bar{l}^{2}-k\right) \bar{a}_{k} & \bar{b}_{k}
\end{array}\right) ; k \geq 1 .
\end{gathered}
$$

Observe que se $k \geq m$ então $\bar{a}_{k}=\bar{b}_{k}=0$. Logo, $\frac{\partial F_{k}}{\partial x_{0}}(\bar{x})=0 \forall k \geq m$.

Por fim,

$$
\begin{gathered}
\frac{\partial F_{0}}{\partial x_{0}}(\bar{x})=\left(\begin{array}{cc}
0 & 0 \\
0 & \bar{a}_{0}
\end{array}\right) ; \\
\frac{\partial F_{0}}{\partial x_{1}}(\bar{x})=\left(\begin{array}{cc}
0 & 1 \\
2 \bar{a}_{1} & 2 \bar{b}_{1}
\end{array}\right) ;
\end{gathered}
$$

e

$$
\frac{\partial F_{0}}{\partial x_{j}}(\bar{x})=\left(\begin{array}{cc}
0 & 0 \\
2 \bar{a}_{j} & 2 \bar{b}_{j}
\end{array}\right), j \geq 2 .
$$

Considere os termos $\frac{\partial F_{k}}{\partial x_{j}}$ em que $k \neq j$ (fora da diagonal). As quatro observações a seguir motiva a construção de uma aproximação para o operador $D F(\bar{x})$, e, consequentemente, uma aproximação para a sua inversa $[D F(\bar{x})]^{-1}$. 
1. Observe que se $k>m$ então $\left(\frac{\partial F_{k}}{\partial x_{j}}\right) \cdot v_{j}=\left(\begin{array}{cc}\bar{a}_{k-j} & -\bar{b}_{k-j} \\ \bar{b}_{k-j} & \bar{a}_{k-j}\end{array}\right) \cdot v_{j} \Rightarrow\left\|\frac{\partial F_{k}}{\partial x_{j}}(\bar{x}) \cdot v_{j}\right\|_{\infty} \leq$ $2 \frac{\|\bar{x}\|_{s} \cdot\|v\|_{s}}{j^{s}|k-j|^{s}}$. Logo, se $j$ é grande, o número $\left\|\frac{\partial F_{k}}{\partial x_{j}}(\bar{x}) \cdot v_{j}\right\|_{\infty}$ fica pequeno. Tomando $m$ grande e $k>m$, se $j$ é pequeno, temos que $|k-j|>m-j$ é grande, ou seja, $\left\|\frac{\partial F_{k}}{\partial x_{j}}(\bar{x}) \cdot v_{j}\right\|_{\infty}$ é pequeno.

Resumindo, tomando $m$ suficientemente grande, se $k>m$, então $\left\|\frac{\partial F_{k}}{\partial x_{j}}(\bar{x}) \cdot v_{j}\right\|_{\infty}$ é pequeno qualquer que seja $j \geq 0, j \neq k$. Ou seja, nas linhas de maior ordem da matriz $\left(\frac{\partial F_{k}}{\partial x_{j}}\right)_{k \geq 0, j \geq 0}$ os elementos fora da diagonal são pouco relevantes para o cálculo de $D F(\bar{x}) \cdot v$.

2. Por outro lado, se $k \leq m$ e $j$ é grande, como os elementos $v_{j}$ tendem a zero e as componentes de $\left(\begin{array}{cc}\bar{a}_{k-j}+\bar{a}_{k+j} & \bar{b}_{k+j}-\bar{b}_{k-j} \\ \bar{b}_{k-j}+\bar{b}_{k+j} & \bar{a}_{k-j}-\bar{a}_{k+j}\end{array}\right) ; k \geq 0, j \geq 0$, são limitadas, segue que os termos de maior ordem das $m$ primeiras linhas também são pouco relevantes para o cálculo de $D F(\bar{x}) \cdot v$.

3. Segue dos dois itens acima que, dentre as componentes $\frac{\partial F_{k}}{\partial x_{j}}(\bar{x})$ da matrix de $D F(\bar{x})$ tais que $k \geq m$ e $j \geq m$, as mais relevantes são aquelas que compoem a diagonal, ou seja, as componentes

$$
\frac{\partial F_{k}}{\partial x_{k}}(\bar{x})=\left(k \bar{l}-k^{3} \bar{l}^{3}\right)\left(\begin{array}{cc}
0 & 1 \\
-1 & 0
\end{array}\right)+\left(\begin{array}{cc}
\bar{a}_{0} & 0 \\
0 & \bar{a}_{0}
\end{array}\right) .
$$

4. Por fim, note que o bloco formado pelas primeiras $m$ linhas e $m$ colunas é exatamente a jacobiana de $F^{m}$ no ponto $\bar{c}$, a qual podemos calcular usando o computador.

Considerando a aproximação

$$
\Lambda_{k}(\bar{x})=\left(k \bar{l}-k^{3} \bar{l}^{3}\right)\left(\begin{array}{cc}
0 & 1 \\
-1 & 0
\end{array}\right) \approx \frac{\partial F_{k}}{\partial x_{k}}(\bar{x}), k \geq m,
$$

segue das quatro observações acima que o operador

$$
A^{\dagger}=\left(\begin{array}{cccc}
J F^{m}(\bar{c}) & & & \\
& \Lambda_{m}(\bar{x}) & & \\
& & \Lambda_{m+1}(\bar{x}) & \\
& & & \ddots
\end{array}\right), \quad A^{\dagger} v=\left(\begin{array}{c}
J F^{m}(\bar{c}) v^{m} \\
\Lambda_{k}(\bar{x}) \cdot v_{m} \\
\Lambda_{k}(\bar{x}) \cdot v_{m+1} \\
\vdots
\end{array}\right)
$$

onde $v^{m}=\left(v_{0}, v_{1}, \ldots, v_{m-1}\right)$, é uma aproximação do operador $D F(\bar{x})$, a qual é tão melhor quanto maior for o valor de $m$. Este operador é computacionalmente viável. De fato, dado $v \in \Omega^{s}$ e $k>0$, é possível obter a $k$-ésima componente de $A^{\dagger} v$, que denotaremos por $\left(A^{\dagger} v\right)_{k}$, com um número finito de cálculos, e, mais importante que isso, denotando por $A^{m}$ a inversa numérica de $D F^{m}(\bar{c}), A^{m} \approx\left(D F^{m}(\bar{c})\right)^{-1}$, segue que o operador

$$
A=\left(\begin{array}{cccc}
A^{m} & & & \\
& {\left[\Lambda_{m}(\bar{x})\right]^{-1}} & & \\
& & {\left[\Lambda_{m+1}(\bar{x})\right]^{-1}} & \\
& & \ddots
\end{array}\right)
$$


é uma aproximação para a inversa de $D F(\bar{x})$. De fato,

$$
A \approx\left(A^{\dagger}\right)^{-1} \approx[D F(\bar{x})]^{-1} \text {. }
$$

A maior vantagem é que o operador $A$ assim definido pode ser facilmente manipulado no computador.

Resta provar que, de fato, existe $m>0$ tal que as inversas $\left[\Lambda_{k}(\bar{x})\right]^{-1}$ de fato existem para todo $k \geq m$. Para ver isto, note que, para $k \geq m$, $\operatorname{det}\left(\Lambda_{k}(\bar{x})\right)=\left(k \bar{l}-k^{3} \bar{l}^{3}\right)^{2}$. Logo, se $\left(k^{3} \bar{l}^{3}-k \bar{l}\right)>0$, ou seja, se $k>\frac{1}{|\bar{l}|}$, então $\Lambda_{k}(\bar{x})$ é inversível.

No entanto, o valor de $m$ teve de ser fixado no momento em que $\bar{c}$ foi calculado, na primeira etapa do método. Logo, pode ocorrer que o valor de $m$ escolhido seja tal que $m<\frac{1}{\bar{l}}$. Nesse caso, tome $m=\left\lceil\frac{1}{\bar{l}}\right\rceil=$ menor inteiro maior que $\frac{1}{|\bar{l}|}$, e extenda $\bar{c}$ a um elemento de $\mathbb{R}^{2 m}$ tomando as componentes restantes iguais a zero. Por exemplo, suponha que a princípio tomamos $m=10$ e o Método de Newton fornece $\bar{l}=0.01$, de modo que $10=m<\frac{1}{\bar{l}}=100$. Nesse caso, passamos a considerar $m=100$ e modificamos a aproximação obtida no Método de Newton para $\left(\bar{c}, 0_{90}\right)$, onde $0_{90}$ é o elemento nulo de $\mathbb{R}^{90}$. Note que, pelo que foi dito acima, as inversas $\left[\Lambda_{k}(\bar{x})\right]^{-1}$ existem para $m \geq 100$ e $\bar{x}=\left(\bar{c}, 0_{90}, 0_{\infty}\right)$. Logo, implementamos o operador $A$ usando $m=100 \mathrm{com}$ a nova aproximação numérica dada por $\left(\bar{c}, 0_{90}\right) \in \mathbb{R}^{200}$.

Encerramos esta seção com um teorema que estabelece um operador de ponto fixo a partir do qual buscaremos pelas raízes de $F$. Como já foi dito, queremos provar que este operador é uma contração numa vizinhança da aproximação numérica.

Teorema 3.5.1. Sejam $m>0, s>2, \bar{c}=\left(\bar{l}, \bar{a}_{0}, \bar{a}_{1}, \bar{b}_{1}, \ldots, \bar{b}_{m-1}\right) \in \mathbb{R}^{2 m}$ tal que $F^{m}(\bar{c}) \approx 0$ $e \bar{x}=\left(\bar{c}, 0_{\infty}\right) \in \Omega^{s}$.

Suponha que $m>\frac{1}{\bar{l}}$. Então

$$
A=\left(\begin{array}{cccc}
A^{m} & & & \\
& {\left[\Lambda_{m}(\bar{x})\right]^{-1}} & & \\
& & {\left[\Lambda_{m+1}(\bar{x})\right]^{-1}} & \\
& & \ddots
\end{array}\right)
$$

é tal que $A: \Omega^{s-3} \rightarrow \Omega^{s}$. Portanto, $T(x)=x-A F(x)$ é tal que

$$
T: \Omega^{s} \rightarrow \Omega^{s} .
$$

Demonstração. Para $k>m$ podemos escrever

$$
\begin{gathered}
\Lambda_{k}(\bar{x})=\left(\begin{array}{cc}
0 & \left(k \bar{l}-k^{3} \bar{l}^{3}\right) \\
-\left(k \bar{l}-k^{3} \bar{l}^{3}\right) & 0
\end{array}\right) \Rightarrow \\
\Rightarrow\left[\Lambda_{k}(\bar{x})\right]^{-1}=\frac{1}{\left(k^{3} \bar{l}^{3}-k \bar{l}\right)^{2}}\left(\begin{array}{cc}
0 & -\left(k \bar{l}-k^{3} \bar{l}^{3}\right) \\
\left(k \bar{l}-k^{3} \bar{l}^{3}\right) & 0
\end{array}\right) .
\end{gathered}
$$

Logo, dado $\left(v_{j}\right)_{j \geq 0} \in \Omega^{s-3}$ temos

$$
\left\|\left[\Lambda_{k}(\bar{x})\right]^{-1} \cdot v_{k}\right\|_{\infty} \leq \frac{1}{\left|\left(k \bar{l}-k^{3} \bar{l}^{3}\right)\right|} \cdot\left\|v_{k}\right\|_{\infty} \leq \frac{1}{k^{3}} \cdot\left(\frac{1}{\bar{l}^{3}-\frac{\bar{l}}{m^{2}}}\right) \cdot\left\|v_{k}\right\|_{\infty}=: \frac{C_{m}}{k^{3}} \cdot\left\|v_{k}\right\|_{\infty} .
$$

Logo,

$$
\left\|\left[\Lambda_{k}(\bar{x})\right]^{-1} \cdot v_{k}\right\|_{\infty} \cdot k^{s} \leq \frac{C_{m}}{k^{3}} \cdot \frac{\|v\|_{s-3}}{k^{s-3}} \cdot k^{s}=C_{m} \cdot\|v\|_{s-3}, \quad \forall k \geq m,
$$

o que implica que $A \cdot v \in \Omega^{s}$, como queriamos. 


\subsection{Polinômios Radiais}

Em todo o restante deste trabalho, dado $x=\left(x_{0}, x_{1}, x_{2}, \ldots\right)$ com $x_{i} \in \mathbb{R}$ denote por $|x|=\left(\left|x_{0}\right|,\left|x_{1}\right|,\left|x_{2}\right|, \ldots\right)$. Se $m \in \mathbb{N}$ e $x=\left(x_{0}, x_{1}, x_{2}, \ldots\right) \in \Omega^{s}, \operatorname{com} x_{i} \in \mathbb{R}^{n}, i=0,1,2, \ldots$, denote por $x^{m}=\left(x_{0}, x_{1}, \ldots, x_{m-1}\right) \in \mathbb{R}^{n m}$. Por fim, dada uma matriz real $M_{m \times n}$, denote por $|\mathrm{M}|$ a matriz cujas componentes são os valores absolutos das componentes de $M$ e por $\leq_{\text {c.c. }}$ a desigualdade componente a componente entre matrizes reais.

O método dos polinômos radiais fornece condições verificáveis pelo computador que garantem as hipóteses do teorema do ponto fixo de Banach. A seguir, introduzimos um teorema que fornece tais condições.

Teorema 3.6.1. Sejam $T(x)=x-A F(x), x \in \Omega^{s}$, onde $F$ é dada por (3.13) e A : $\Omega^{s-3} \rightarrow \Omega^{s}$ é uma aplicação linear invertível. Suponha que existam $Z(r)$ e $Y$ em $\Omega^{s}$ tais que

$$
\begin{gathered}
\sup _{u, v \in B(0,1)}\left|(D T(\bar{x}+r u) \cdot r v)_{k}\right| \leq_{c . c} Z_{k}(r), \\
\left|(T(\bar{x})-\bar{x})_{k}\right| \leq_{c . c} Y_{k} .
\end{gathered}
$$

Nessas condições, se existir $r>0$ tal que

$$
\|Z(r)+Y\|_{s}<r
$$

então $T$ possui um único ponto fixo $\hat{x}$ na bola $B(\bar{x}, r)$. Em particular, $F(\hat{x})=0$ e (3.1) possui uma órbita periódica.

Demonstração. Nossa estratégia é obter $r>0$ tal que o operador $T$ dado pelo Teorema 3.5.1 seja uma contração numa bola $B_{\Omega^{s}}[\bar{x}, r]$. Em particular $T$ deve deixar $B_{\Omega^{s}}[\bar{x}, r]$ invariante, ou seja, $T\left(B_{\Omega^{s}}[\bar{x}, r]\right) \subset B_{\Omega^{s}}[\bar{x}, r]$ Desse modo, é necessário que exista $r>0$ tal que

$$
\|T(x)-\bar{x}\|_{s} \leq r, \quad \forall x \in B_{\Omega^{s}}[\bar{x}, r] .
$$

Nesse sentido, para cada $k \geq 0$, podemos escrever:

$$
\left\|[T(x)-\bar{x}]_{k}\right\|_{\infty} \leq\left\|T_{k}(x)-T_{k}(\bar{x})\right\|_{\infty}+\left\|T_{k}(\bar{x})-\bar{x}_{k}\right\|_{\infty} .
$$

Como $T_{k}: B_{\Omega^{s}}[\bar{x}, r] \rightarrow \mathbb{R}^{2}$, podemos definir

$$
\begin{gathered}
g_{k}:[0,1] \rightarrow \mathbb{R}^{2} \\
g_{k}(t)=T_{k}(\bar{x}+t(x-\bar{x})) .
\end{gathered}
$$

Pela desigualdade do valor médio, existe $\bar{t} \in[0,1]$ tal que:

$$
\begin{gathered}
\left\|T_{k}(x)-T_{k}(\bar{x})\right\|_{\infty}=\left\|g_{k}(1)-g_{k}(0)\right\|_{\infty} \leq\left\|g_{k}^{\prime}(\bar{t}) \cdot 1\right\|_{\infty}=\left\|D T_{k}(\bar{x}+\bar{t}(x-\bar{x}))(x-\bar{x})\right\|_{\infty}= \\
=\left\|D T_{k}(\bar{x}+\bar{t}(x-\bar{x})) \frac{(x-\bar{x})}{\|x-\bar{x}\|_{s}}\right\|_{\infty}\|x-\bar{x}\|_{s} \leq \\
\leq \sup _{u, v \in B_{\Omega^{s}}[0,1]}\left\|D T_{k}(\bar{x}+r u) \cdot v\right\|_{\infty} r .
\end{gathered}
$$

Substituindo (3.38) em (3.37), obtemos:

$$
=\left\|[T(x)-\bar{x}]_{k}\right\|_{\infty} \leq \sup _{u, v \in B_{\Omega^{s}}[0,1]}\left\|D T_{k}(\bar{x}+r u) \cdot v\right\|_{\infty} r+\left\|T_{k}(\bar{x})-\bar{x}_{k}\right\|_{\infty} .
$$


Sejam $Z=Z(r), Y \in \Omega^{s}$ tais que

$$
\sup _{u, v \in B_{\Omega^{s}}(0,1)} \mid\left[D T_{k}(\bar{x}+r u) \cdot r v \mid \leq_{c . c} Z_{k}(r), \text { para todo } k \geq 0\right.
$$

$\mathrm{e}$

$$
\left|T_{k}(\bar{x})-\bar{x}_{k}\right| \leq_{c . c} Y_{k}, \quad \text { para todo } k \geq 0 .
$$

Suponha que $\|Z(r)\|_{s}+\|Y\|_{s}=\|Z(r)+Y\|_{s}<r$. Então, por (3.39), segue que

$$
\left\|[T(x)-\bar{x}]_{k}\right\|_{\infty} k^{s} \leq\left\|Z_{k}(r)\right\|_{\infty} k^{s}+\left\|Y_{k}\right\|_{\infty} k^{s}
$$

o que implica que

$$
\|T(x)-\bar{x}\|_{s} \leq\left\|Z_{k}(r)\right\|_{s}+\left\|Y_{k}\right\|_{s}<r
$$

ou seja,

$$
T(B(\bar{x}, r)) \subset B(\bar{x}, r)
$$

como queríamos provar.

A existência das cotas $Z(r)$ e $Y$ com as propriedades acima garantem ainda que $T$ é uma contração. De fato, usando as mesmas ideias apresentadas acima, dados $x, y \in$ $B_{\Omega^{s}}[\bar{x}, r]$, obtemos:

$$
\begin{aligned}
\left\|T_{k}(x)-T_{k}(y)\right\|_{\infty} \leq & \left(\sup _{u, v \in B_{\Omega^{s}}(0,1)}\left\|\left[D T_{k}(\bar{x}+r u) \cdot v \|_{\infty}\right)\right\| x-y \|_{s}\right. \\
& \leq \frac{\left\|Z_{k}(r)\right\|_{\infty}}{r}\|x-y\|_{s}
\end{aligned}
$$

Como $\|Z(r)\|_{s} \leq\|Z(r)\|_{s}+\|Y\|_{s}<r$, segue que $\frac{\|Z(r)\|_{s}}{r}<1$. Ou seja, $T$ é uma contração.

Observação 3.6.1. Denotando $Z_{k}(r)=\left(Z_{k, 1}(r), Z_{k, 2}(r)\right)$ e $Y_{k}=\left(Y_{k, 1}, Y_{k, 2}\right)$ a condição (3.35) é equivalente a

$$
Z_{k, i}(r)+Y_{k, i}-\frac{r}{\omega_{k}^{s}}<0, i=1,2, k \geq 0
$$

Sendo assim, as desigualdades dadas por (3.40) fornecem condições suficientes para que $T$ possua pontos fixos, ou ainda, para que $F$ possua raízes. A verificação dessas condições depende de conhecermos cotas $Z(r)$ e $Y$ com as propriedades (3.33) e (3.34). Mostraremos que as cotas $Z(r)$ e $Y$ podem ser tomadas de forma que as expressões $Z_{k, i}(r)+Y_{k, i}-\frac{r}{\omega_{k}^{s}}$ sejam polinômios em $r$. O que facilitará a busca por $r$ satisfazendo as desigualdades (3.40). Os polinômios

$$
p_{k, i}(r)=Z_{k, i}(r)+Y_{k, i}-\frac{r}{\omega_{k}^{s}}
$$

$i=1,2, k \geq 0$, serão denominados Polinômios Radiais, tendo em vista que sua variável $r$ diz respeito a raios de bolas fechadas em $\Omega^{s}$.

Resumindo, o Teorema 3.6.1 afirma que a existência de $r>0$ tal que $p_{k, i}(r)<0$ para todo $k \geq 0$ e $i \in\{1,2\}$ implica a existência de órbitas periódicas para a equação (3.1).

No que segue, passamos à deteminação das cotas $Z(r)$ e $Y$. 


\subsubsection{Cálculo de $Z(r)$}

Fixe $m>\frac{1}{|l|}$. Para $k<m$ temos:

$$
\begin{gathered}
(D T(\bar{x}+r u) \cdot r v)^{m}=r v^{m}-A^{m}\left(\begin{array}{c}
D F_{0}(\bar{x}+r u) \cdot r v \\
\vdots \\
D F_{m-1}(\bar{x}+r u) \cdot r v
\end{array}\right)= \\
=r v^{m}-A^{m}\left(\begin{array}{c}
D F_{0}(\bar{x}) \cdot r v \\
\vdots \\
D F_{m-1}(\bar{x}) \cdot r v
\end{array}\right)-A^{m}\left(\begin{array}{c}
D F_{0}(\bar{x}+r u) \cdot r v-D F_{0}(\bar{x}) \cdot r v \\
\vdots \\
D F_{m-1}(\bar{x}+r u) \cdot r v-D F_{m-1}(\bar{x}) \cdot r v
\end{array}\right) .
\end{gathered}
$$

Além disso, definindo $S^{m}: \mathbb{R}^{2 m} \rightarrow \Omega^{s}, S(x)=\left(x, 0_{\infty}\right)$ (a qual é claramente linear), temos:

$$
F_{k}^{m}(x)=F_{k}\left(x, 0_{\infty}\right)=F_{k} \circ S^{m}(x)
$$

Derivando, obtemos:

$$
\begin{gathered}
D F_{k}^{m}(\bar{c}) \cdot v^{m}=D F_{k}\left(\bar{c}, 0_{\infty}\right) \cdot\left(v^{m}, 0_{\infty}\right)= \\
=D F_{k}\left(\bar{c}, 0_{\infty}\right) \cdot\left[v-\left(0, \ldots 0, v_{m}, v_{m+1}, v_{m+2}, \ldots\right)\right]= \\
=D F_{k}(\bar{x}) \cdot v-D F_{k}(\bar{x}) \cdot\left(0, \ldots 0, v_{m}, v_{m+1}, v_{m+2}, \ldots\right) .
\end{gathered}
$$

Logo,

$$
D F_{k}(\bar{x}) \cdot v=D F_{k}^{m}(\bar{c}) \cdot v^{m}+D F_{k}(\bar{x}) \cdot\left(0, \ldots 0, v_{m}, v_{m+1}, v_{m+2}, \ldots\right) .
$$

Substituindo esta identidade na expressão de $(D T(\bar{x}+r u) \cdot r v)^{m}$ acima, obtemos

$$
\begin{aligned}
(D T(\bar{x}+r u) \cdot r v)^{m}= & r v^{m}-A^{m} D F^{m}(\bar{c}) \cdot r v^{m}-r A^{m}\left(\begin{array}{c}
D F_{0}(\bar{x}) \cdot\left(0, \ldots 0, v_{m}, v_{m+1}, \ldots\right) \\
\vdots \\
D F_{m-1}(\bar{x}) \cdot\left(0, \ldots 0, v_{m}, v_{m+1}, \ldots\right)
\end{array}\right)- \\
& -A^{m}\left(\begin{array}{c}
D F_{0}(\bar{x}+r u) \cdot r v-D F_{0}(\bar{x}) \cdot r v \\
\vdots \\
D F_{m-1}(\bar{x}+r u) \cdot r v-D F_{m-1}(\bar{x}) \cdot r v
\end{array}\right)
\end{aligned}
$$

Note que $D F_{0}(\bar{x})\left(0, \ldots, 0, v_{m}, v_{m+1}, \ldots\right)=0$ e, para $1 \leq k \leq m-1$, temos

$$
\left\|D F_{k}(\bar{x})\left(0, \ldots, 0, v_{m}, v_{m+1}, \ldots\right)\right\|_{\infty} \leq \sum_{j=m}^{m+k-1} \frac{\left|\bar{a}_{j-k}\right|+\left|\bar{b}_{j-k}\right|}{j^{s}}=: \beta_{k} .
$$

Defina $B_{0}=\left(\begin{array}{l}0 \\ 0\end{array}\right)$ e $B_{k}=\left(\begin{array}{c}\beta_{k} \\ \beta_{k}\end{array}\right), k \geq 1$.

Por outro lado,

$$
\left|r v^{m}-A^{m} D F^{m}(\bar{c}) \cdot r v^{m}\right| \leq_{c . c .} r\left|\left(I d-A^{m} D F^{m}(\bar{c})\right)\right| w^{m},
$$

onde $w=\left\{\frac{1}{\omega_{k}^{s}}\right\}_{k=0}^{\infty}$. 
Observando (3.41), (3.42) e (3.43), percebemos que nos falta apenas limitar os termos da forma

$$
D F_{k}(\bar{x}+r u) \cdot r v-D F_{k}(\bar{x}) \cdot r v, \quad \text { para } 0 \leq k \leq m-1 .
$$

Para isso, note que

$$
\left[D F_{k}(\bar{x}+r u)-D F_{k}(\bar{x})\right] \cdot r v=\sum_{j=0}^{\infty}\left[\frac{\partial F_{k}}{\partial x_{j}}(\bar{x}+r u)-\frac{\partial F_{k}}{\partial x_{j}}(\bar{x})\right] \cdot r v_{j} .
$$

As expressões

$$
\left[\frac{\partial F_{k}}{\partial x_{j}}(\bar{x}+r u)-\frac{\partial F_{k}}{\partial x_{j}}(\bar{x})\right] \cdot r v_{j}
$$

podem ser estimadas a partir das fórmulas que vão de (3.26) a (3.30), lembrando que $\left\|u_{k}\right\|_{\infty} \leq \frac{1}{\omega_{k}^{s}}$ e $\left\|v_{k}\right\|_{\infty} \leq \frac{1}{\omega_{k} k^{s}}$ para todo $k \geq 0$ e recorrendo às cotas $\sigma_{k}(s)$ dadas pelo Teorema 3.1.2. Para facilitar as estimativas consideramos $0 \leq r \leq 1$, obtendo:

Para $k \geq 1$ :

$$
\begin{gathered}
\left\|\left[D F_{k}(\bar{x}+r u)-D F_{k}(\bar{x})\right] \cdot r v\right\|_{\infty} \leq\left[3 k^{3}(2|\bar{l}|+1)\left(\left|\bar{a}_{k}\right|+\left|\bar{b}_{k}\right|\right)\right] \cdot r^{2}+ \\
+\left[4 \sum_{j=0}^{\infty} \omega_{k-j}^{-s} \omega_{k}^{-s}+\frac{\left(6 \bar{l}^{2}+9|\bar{l}|+4\right) k^{3}+2 k+1}{\omega_{k}^{s}}\right] \cdot r^{2} \leq \\
\leq \underbrace{\left[3 k^{3}(2|\bar{l}|+1)\left(\left|\bar{a}_{k}\right|+\left|\bar{b}_{k}\right|\right)+4 \frac{\sigma_{k}(s)}{\omega_{k}^{s}}+\frac{\left(6 \bar{l}^{2}+9|\bar{l}|+4\right) k^{3}+2 k+1}{\omega_{k}^{s}}\right]}_{\eta_{k}} \cdot r^{2}
\end{gathered}
$$

Para $k=0$ :

$$
\left\|\left[D F_{0}(\bar{x}+r u)-D F_{0}(\bar{x})\right] \cdot r v\right\|_{\infty} \leq\left[4 \sum_{j=0}^{\infty} \omega_{k-j}^{-s} \omega_{k}^{-s}+1\right] \cdot r^{2} \leq \underbrace{\left[4 \sigma_{0}(s)\right]}_{\eta_{0}} \cdot r^{2} .
$$

Defina $E_{k}=\left(\begin{array}{c}\eta_{k} \\ \eta_{k}\end{array}\right), 0 \leq k \leq m-1$. Substituindo-se as estimativas (3.42), (3.43), (3.44) e (3.45) em (3.41) obtem-se:

$$
\begin{aligned}
\left|(D T(\bar{x}+r u) \cdot r v)^{m}\right| \leq_{c . c .} & {\left[\left|\left(I d-A^{m} D F^{m}(\bar{c})\right)\right| \cdot w^{m}+\left|A^{m}\right| \cdot\left(\begin{array}{c}
B_{0} \\
\vdots \\
B_{m-1}
\end{array}\right)\right] \cdot r+} \\
& +\left|A^{m}\right| \cdot\left(\begin{array}{c}
E_{0} \\
\vdots \\
E_{m-1}
\end{array}\right) \cdot r^{2}
\end{aligned}
$$


Logo, podemos tomar

$$
\begin{gathered}
{[Z(r)]^{m}=\left[\left|\left(I d-A^{m} D F^{m}(\bar{c})\right)\right| \cdot w^{m}+\left|A^{m}\right| \cdot\left(\begin{array}{c}
B_{0} \\
\vdots \\
B_{m-1}
\end{array}\right)\right] \cdot r+} \\
+\left|A^{m}\right| \cdot\left(\begin{array}{c}
E_{0} \\
\vdots \\
E_{m-1}
\end{array}\right) \cdot r^{2} .
\end{gathered}
$$

Para $k \geq m$, temos:

$$
\begin{gathered}
D T_{k}(\bar{x}+r u) \cdot r v=r v_{k}-\left[\Lambda_{k}(\bar{x})\right]^{-1} \cdot \sum_{j=0}^{\infty} \frac{\partial F_{k}}{\partial x_{j}}(\bar{x}+r u) \cdot r v_{j}= \\
=r v_{k}-\left[\Lambda_{k}(\bar{x})\right]^{-1} \cdot \sum_{j=0}^{\infty} \frac{\partial F_{k}}{\partial x_{j}}(\bar{x}) \cdot r v_{j}-\left[\Lambda_{k}(\bar{x})\right]^{-1} \sum_{j=0}^{\infty}\left[\frac{\partial F_{k}}{\partial x_{j}}(\bar{x}+r u)-\frac{\partial F_{k}}{\partial x_{j}}(\bar{x})\right] \cdot r v_{j}= \\
=-\left[\Lambda_{k}(\bar{x})\right]^{-1} \cdot \sum_{j=0}^{\infty} \frac{\partial F_{k}}{\partial x_{j}}(\bar{x}) \cdot r v_{j}-\left[\Lambda_{k}(\bar{x})\right]^{-1} \sum_{j=0}^{\infty}\left[\frac{\partial F_{k}}{\partial x_{j}}(\bar{x}+r u)-\frac{\partial F_{k}}{\partial x_{j}}(\bar{x})\right] \cdot r v_{j}= \\
=-\left[\Lambda_{k}(\bar{x})\right]^{-1} \cdot \sum_{j=1}^{m+k-1}\left(\begin{array}{cc}
\bar{a}_{k-j} & -\bar{b}_{k-j} \\
\bar{b}_{k-j} & \bar{a}_{k-j}
\end{array}\right) r v_{j}-\left[\Lambda_{k}(\bar{x})\right]^{-1} \cdot\left[D F_{k}(\bar{x}+r u)-D F_{k}(\bar{x})\right] \cdot r v .
\end{gathered}
$$

Logo, para $k \geq m$, temos

$$
\left\|D T_{k}(\bar{x}+r u) \cdot r v\right\|_{\infty} \leq\left[\frac{C_{m}}{k^{3}} \sum_{j=1}^{m+k-1} \frac{\left|\bar{a}_{k-j}\right|+\left|\bar{b}_{k-j}\right|}{j^{s}}\right] \cdot r+\left[\frac{C_{m}}{k^{3}} \eta_{k}\right] \cdot r^{2}
$$

e podemos tomar

$$
Z_{k}(r)=\left[\frac{C_{m}}{k^{3}} \sum_{j=1}^{m+k-1} \frac{\left|\bar{a}_{k-j}\right|+\left|\bar{b}_{k-j}\right|}{j^{s}}\right] \cdot r+\left[\frac{C_{m}}{k^{3}} \eta_{k}\right] \cdot r^{2}, k \geq m .
$$

Observação 3.6.2. Lembremos que, de acordo com o Teorema 3.6.1, estamos interessados em encontrar cotas $Z(r)$ e $Y$ no conjunto $\Omega^{s}$ tais que $\left\|Z_{k}(r)+Y_{k}\right\|_{\infty} \leq \frac{r}{\omega_{k}^{s}}$ para todo $k \geq 0$. Mas isso fornece uma quantidade infinita de condições a serem verificadas, o que é impossivel do ponto de vista computacional.

Porém, mostraremos a seguir que, dado $M \geq 6$, podemos construir um polinômio $Z_{M}(r)$ tal que $Z_{k}(r) \leq_{\text {c.c. }} \frac{Z_{M}(r)}{\omega_{k}^{s}}$ para todo $k \geq M$ e para todo $r>0$. Mostraremos ainda que, para todo $k \geq 2 m$, temos $Y_{k}=0$.

Feito isso, tomando $M \geq 2 m$, podemos escrever

$$
\left\|Z_{k}(r)+Y_{k}\right\|_{\infty} \leq\left\|Z_{M}(r)+Y_{M}\right\|_{\infty}, k \geq M
$$

Com isso, se existir $r>0$ tal que $\left\|Z_{M}(r)+Y_{M}\right\|_{\infty}=\left\|Z_{M}(r)\right\|_{\infty}<1$ teremos, para todo $k \geq M$, que

$$
\left\|Z_{k}(r)+Y_{k}\right\|_{\infty}=\left\|Z_{k}(r)\right\|_{\infty} \leq \frac{\left\|Z_{M}(r)\right\|}{k^{s}} \leq \frac{1}{k^{s}}
$$


Ou seja, a demonstração da existência de órbitas periódicas fica reduzida à verificação de uma quantidade finita de condições, a saber:

$$
\begin{gathered}
\left\|Z_{k}(r)+Y_{k}\right\|_{\infty} \leq \frac{1}{\omega_{k}^{s}}, k \in\{0,1, \ldots, M-1\}, \\
Z_{M}(r)<1 .
\end{gathered}
$$

Como prometido na observação acima, encerramos esta seção com o seguinte teorema.

Teorema 3.6.2. Seja $M \geq 6$ um natural fixado. Então, para todo $k \geq M$ temos

onde

$$
\left\|Z_{k}(r)\right\|_{\infty} \leq \frac{Z_{M}(r)}{k^{s}}
$$

$$
\begin{aligned}
Z_{M}(r) & =\frac{C_{m}}{M^{3}}\left\{\left|\bar{a}_{0}\right|+\sum_{j=1}^{m-1}\left(\left|\bar{a}_{j}\right|+\left|\bar{b}_{j}\right|\right)\left[1+\frac{1}{\left(1-\frac{j}{M}\right)^{s}}\right]\right\} r+ \\
& +C_{m}\left[\frac{\sigma_{M}(s)}{M^{3}}+\frac{2 M+1}{M^{3}}+6|\bar{l}|^{2}+9|\bar{l}|+4\right] r^{2} .
\end{aligned}
$$

Demonstração. Por (3.45) temos

$$
Z_{k}(r)=\left[\frac{C_{m}}{k^{3}} \sum_{j=1}^{m+k-1} \frac{\left|\bar{a}_{k-j}\right|+\left|\bar{b}_{k-j}\right|}{j^{s}}\right] \cdot r+\left[\frac{C_{m}}{k^{3}} \eta_{k}\right] \cdot r^{2}, k \geq m .
$$

Escrevendo

$$
\sum_{j=1}^{m+k-1} \frac{\left|\bar{a}_{k-j}\right|+\left|\bar{b}_{k-j}\right|}{j^{s}}=\frac{\left|\bar{a}_{0}\right|}{k^{s}}+\frac{1}{k^{s}} \sum_{j=1}^{m-1} \frac{\left|\bar{a}_{j}\right|+\left|\bar{b}_{j}\right|}{\left(1-\frac{j}{k}\right)^{s}}+\frac{1}{k^{s}} \sum_{j=1}^{m-1} \frac{\left|\bar{a}_{j}\right|+\left|\bar{b}_{j}\right|}{(k+j)^{s}},
$$

e observando que $\left(1-\frac{j}{k}\right)^{s} \leq\left(1-\frac{j}{M}\right)^{s}$ para todo $k \geq M$ e que $\frac{1}{(k+j)^{s}} \leq \frac{1}{k^{s}}$ para todo $k>0$, segue a estimativa para o coeficiente de $r$. A estimativa para o coeficiente de $r^{2}$ segue da expressão de $\eta_{k}$ para $k \geq M$ dada em (3.41) e de que $\sigma_{k}(s) \leq \sigma_{M}(s)$ para todo $k \geq M$.

\subsubsection{Cálculo de $Y$}

Queremos encontrar um limitante $Y=\left(Y_{k}\right)_{k \geq 0}$ satisfazendo (3.34).

Seja $x=\left(x_{k}\right)_{k \geq 0} \in \Omega^{s}, x_{k}=\left(x_{k, 1}, x_{k, 2}\right)$ com $x_{k}=0 \forall k \geq m$. Lembremos que

$$
F_{k}(x)=\frac{1}{2} \sum_{k_{1}+k_{2}=k}\left(x_{k_{1}} x_{k_{2}}\right)+i k l x_{k}\left(k^{2} l^{2}-1\right)-\delta_{0, k} \lambda^{2}, k \in \mathbb{Z},
$$

onde consideramos $x_{-k}=\left(x_{k, 1},-x_{k, 2}\right)$ para $k>0$.

Note que, se $k \geq 2 m$ e $k_{1}+k_{2}=k$, então, ou $\left|k_{1}\right| \geq m$, ou $\left|k_{2}\right| \geq m$, pois, se $\left|k_{1}\right|<m$ e $\left|k_{2}\right|<m$, então, $k=k_{1}+k_{2} \leq\left|k_{1}\right|+\left|k_{2}\right|<2 m \leq k$. Absurdo. Logo, se $k \geq 2 m$, temos que $F_{k}(x)=\frac{1}{2} \sum_{k_{1}+k_{2}=k}\left(x_{k_{1}} x_{k_{2}}\right)+i k l x_{k}\left(k^{2} l^{2}-1\right)$, com $\left|k_{1}\right| \geq m\left(\operatorname{logo} x_{k_{1}}=0\right)$, ou $\left|k_{2}\right| \geq m\left(\operatorname{logo}, x_{k_{2}}=0\right)$. Em ambos os casos temos $F_{k}(x)=0, \forall k \geq 2 m$.

Consequentemente, (mantendo a notação dos paragráfos anteriores) se $k \geq 2 m$ então $T(\bar{x})-\bar{x}=0-0=0$. Restando estimar apenas as $2 m$ primeiras componentes de $T(\bar{x})-\bar{x}$. 
Isso pode ser feito usando o computador, pois $(T(\bar{x})-\bar{x})^{m}=A^{m} F^{m}(\bar{c})$ e, para $k \geq m$, temos

$$
\left\|(T(\bar{x})-\bar{x})_{k}\right\|_{\infty}=\left\|\left[\frac{\partial F_{k}}{\partial x_{k}}(\bar{x})\right]^{-1} \cdot F_{k}(\bar{x})\right\|_{\infty} \leq \frac{C_{m}}{k^{3}}\left\|F_{k}(\bar{x})\right\|_{\infty} .
$$

Com isso, podemos tomar

$$
\begin{gathered}
Y^{m}=A^{m} F^{m}(\bar{c}), \\
Y_{k}=\left(\begin{array}{c}
\frac{C_{m}}{k^{3}}\left\|F_{k}(\bar{x})\right\|_{\infty} \\
\frac{c_{m}}{k^{3}}\left\|F_{k}(\bar{x})\right\|_{\infty}
\end{array}\right), m \leq k \leq 2 m-1, \\
Y_{k}=0, k \geq 2 m .
\end{gathered}
$$

\subsection{Resultados}

Em termos práticos, o método para obtenção de soluções periódicas da equação de Michelson descrito neste trabalho pode ser sintetizado nos seguintes passos:

1. Implementamos a projeção finita $F^{m}(x)$ e a Jacobiana $J F^{m}(x)$ para valores de $m \in \mathbb{N}$ e $x \in \mathbb{R}^{2 m}$ fornecidos pelo usuário.

2. Implementamos o Método de Newton $x_{i+1}=x_{i}-\left[J F^{m}\left(x_{i}\right)\right]^{-1} \cdot F^{m}\left(x_{i}\right)$ e o executamos para diferentes valores iniciais de $x_{0} \in \mathbb{R}^{2 m}$ até ocorrer a convergência para uma raiz aproximada de $F^{m}$.

3. Usamos as aproximações obtidas pelo Método de Newton para implementar as cotas $Z(r)$ e $Y$ obtidas nas Seções 3.6.1 e 3.6.2. Com essas cotas implementadas, podemos construir os polinômios radiais de segundo grau $p_{k, i}(r)=Z_{k, i}(r)+Y_{k, i}-\frac{r}{k^{s}}$ para $0 \leq k \leq 2 m$ e $i \in\{1,2\}$. Em seguida, obtemos as raízes de cada um desses polinômios usando o Software MATLAB. Observe que, como todos os polinômios tem o coeficiente do termo $r^{2}$ positivo, cada um dos polinômios radiais assume valores negativos nos intervalos determinados pelos seus pares de raízes. Assim, se denotarmos por $r_{1, k, i}<r_{2, k, i}$ as raízes dos polinômios $p_{k, i}(r)$, devemos obter $\bar{r}>0$ tal que $\bar{r} \in \cap_{k, i}\left(r_{1, k, i}, r_{2, k, i}\right)$. Desse modo, teremos que $p_{k, i}(\bar{r})<$ 0 , sempre que $0 \leq k \leq 2 m$, e $i \in\{1,2\}$. Em seguida, tomamos o menor valor possível de $\bar{r} \in \cap_{k, i}\left(r_{1, k, i}, r_{2, k, i}\right)$ e verificamos se ocorre a desigualdade

$$
Z_{M}(\bar{r})<1
$$

onde $Z_{M}$ é dado pelo Teorema 3.6.2. Para as órbitas traçadas abaixo obtivemos $\bar{r}$ da ordem de $10^{-5}$ satizfazendo todas essas condições, o que reflete grande proximidade entre a órbita numérica, obtida pelo Método de Newton e a órbita exata.

4. Se os cálculos feitos pelo computador até aqui fossem isentos de erro, pelo Teorema 3.6.1 e pela Observação 3.6.2 já teriamos demonstrado a existência de órbitas periódicas para a equação de Michelson. Porém, não podemos garantir que as implementações retornem exatamente as cotas $Z(r)$ e $Y$ obtidas nas seções (3.6.1) e (3.6.2) e nem que as verificações $p_{k, i}(\bar{r})<0, \forall 0 \leq k \leq 2 m, i \in\{1,2\}$ feitas pelo computador sejam exatas.

Para contornar esse problema, implementamos uma versão dos polinômios radiais usando aritmética de intervalo, de modo que seus coeficientes serão intervalos que 
contém os coeficientes exatos. Também usamos aritmética de intervalo para avaliar esses polinômios "intervalares" no ponto $\bar{r}$ obtido no processo numérico descrito no parágrafo acima. Desse modo, os valores retornados nessas avaliações também são dados por intervalos que contém o resultado exato proveniente da avaliação do polinômio verdadeiro em $\bar{r}$. Feito isso, se todos os intervalos provenientes destas avaliações feitas por aritmética de intervalo estiverem contidos na parte negativa da reta real, com maior razão serão negativas as avaliações dos polinômios exatos no ponto $\bar{r}$, e a existência de uma órbita periódica da equação de Michelson fica finalmente provada.

Tomando $m=12$ e $\lambda=1$ o Método de Newton convergiu com o chute inicial dado na Tabela 3.1, apresentada logo abaixo, fornecendo a solução periódica aproximada $\gamma_{12}(t)=$ $\sum_{k=-12}^{12} \bar{c}_{k} e^{-i k \bar{l} t}$, onde $\bar{l}$ e $\bar{c}_{k}=\bar{a}_{k}+i \bar{b}_{k}, k \geq 0$. Os coeficientes não nulos estão apresentados na Tabela 3.1. Na Figura 3.1 temos um esboço da curva $\gamma_{12}(t)$ :

\begin{tabular}{|l|l|l|}
\hline \multicolumn{2}{|c|}{ Chute inicial } & Solução obtida via Método de Newton \\
\hline$l=0.6377$ & $b_{1}=0.6761$ & $\bar{l}=0.3964$ \\
\hline$a_{0}=0.9577$ & $b_{2}=0.6718$ & $\bar{b}_{1}=0.2594$ \\
\hline$a_{1}=0.2407$ & $b_{3}=0.0680$ & $\bar{b}_{2}=0.8701$ \\
\hline$a_{2}=0.2891$ & $b_{4}=0.2240$ & $\bar{b}_{3}=-0.4002$ \\
\hline$a_{3}=0.6951$ & $b_{5}=0.8444$ & $\bar{b}_{4}=-0.1069$ \\
\hline$a_{4}=0.2548$ & $b_{6}=0.7805$ & $\bar{b}_{5}=0.0639$ \\
\hline$a_{5}=0.6678$ & $b_{7}=0.0067$ & $\bar{b}_{6}=-4.0728 \times 10^{-4}$ \\
\hline$a_{6}=0.3445$ & $b_{8}=0.3868$ & $\bar{b}_{7}=-0.0053$ \\
\hline$a_{7}=0.6753$ & $b_{9}=0.0012$ & $\bar{b}_{8}=7.5217 \times 10^{-4}$ \\
\hline$a_{8}=0.6022$ & $b_{10}=0.4243$ & $\bar{b}_{9}=2.6363 \times 10^{-4}$ \\
\hline$a_{9}=0.9160$ & $b_{11}=0.7702$ & $\bar{b}_{10}=-8.4356 \times 10^{-5}$ \\
\hline$a_{10}=0.4624$ & $a_{11}=0.4609$ & $\bar{b}_{11}=-5.6411 \times 10^{-6}$ \\
\hline
\end{tabular}

Tabela 3.1: Chute inicial gerado aleatoriamente pelo MATLAB e solução obtida pelo Método de Newton com esse chute inicial.

Teorema 3.7.1. Com a notação acima, seja $\bar{c}=\left(\bar{l}, \bar{c}_{0}, \ldots, \bar{c}_{11}\right)$. Para $s=2,1$ e $r=$ $7.7225 \times 10^{-4}$, existe único $\hat{c}=\left(\hat{l}, \hat{c}_{0}, \hat{c}_{1}, \ldots\right) \in B_{\Omega^{s}}[\bar{c}, r]$ tal que $F(\hat{c})=0$, ou equivalentemete, $\gamma(t)=\sum_{k \in \mathbb{Z}} \hat{c}_{k} e^{-i k \hat{l} t}$, com $\hat{c}_{-k}=\hat{c}_{k}, k \geq 0$, é uma solução periódica da equação de Michelson.

A partir da solução apresentada acima, podemos repetir todo o processo para calcular uma curva de soluções periódicas conforme variamos o parâmetro $\lambda$. Para isso, variamos o parâmetro $\lambda$ em 0.1 unidades, obtendo um novo problema. Usamos a solução acima como chute inicial para o Método de Newton associado a esse novo problema, obtendo uma nova solução periódica para a nova equação. Continuando com a variação de $\lambda$ obtemos uma curva de soluções periódicas. Na Figura 3.2 mostramos a curva de soluções obtida a partir da solução dada pelo Teorema 3.7.1. Nela podemos visualizar, no mesmo plano, as soluções da equação (3.1) para diferentes valores do parâmetro $\lambda$.

Aplicando o mesmo processo descrito acima com diferentes chutes iniciais aleatórios, obtivemos outras soluções periódicas para o valor de parâmetro $\lambda=1$. Para cada uma dessas soluções pudemos variar o parâmetro $\lambda$ para obter curvas de soluções periódicas. Nas Figuras seguintes apresentamos duas dessas curvas de soluções. 


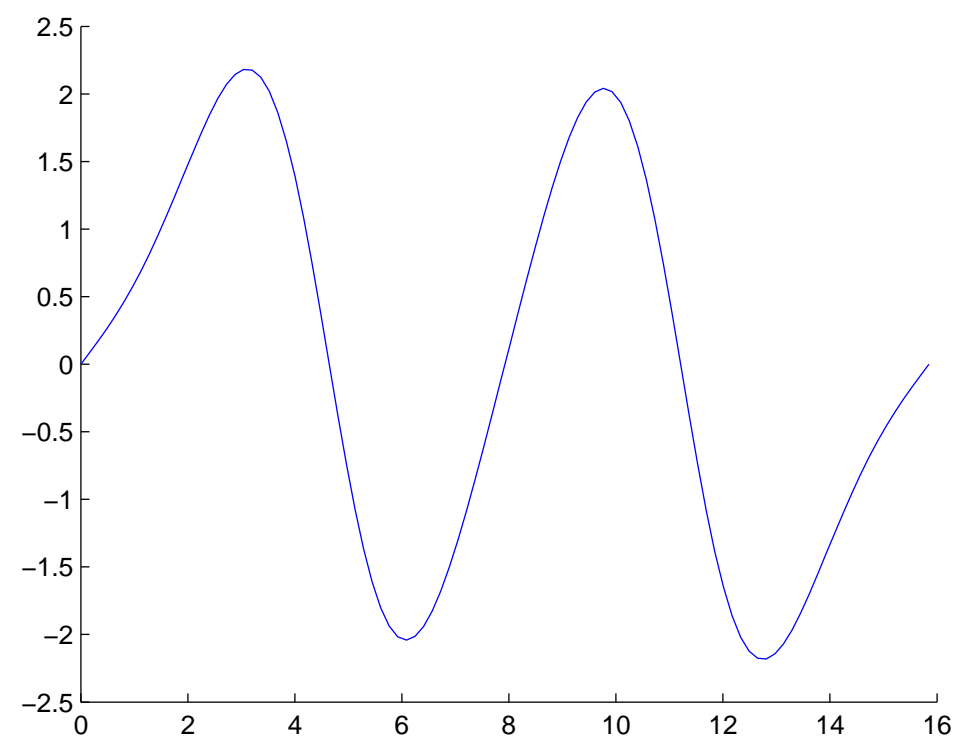

Figura 3.1: Solução periódica da equação (3.1) obtida via Método de Newton, com período $p=2 \pi / \bar{l} \approx 15,85$.

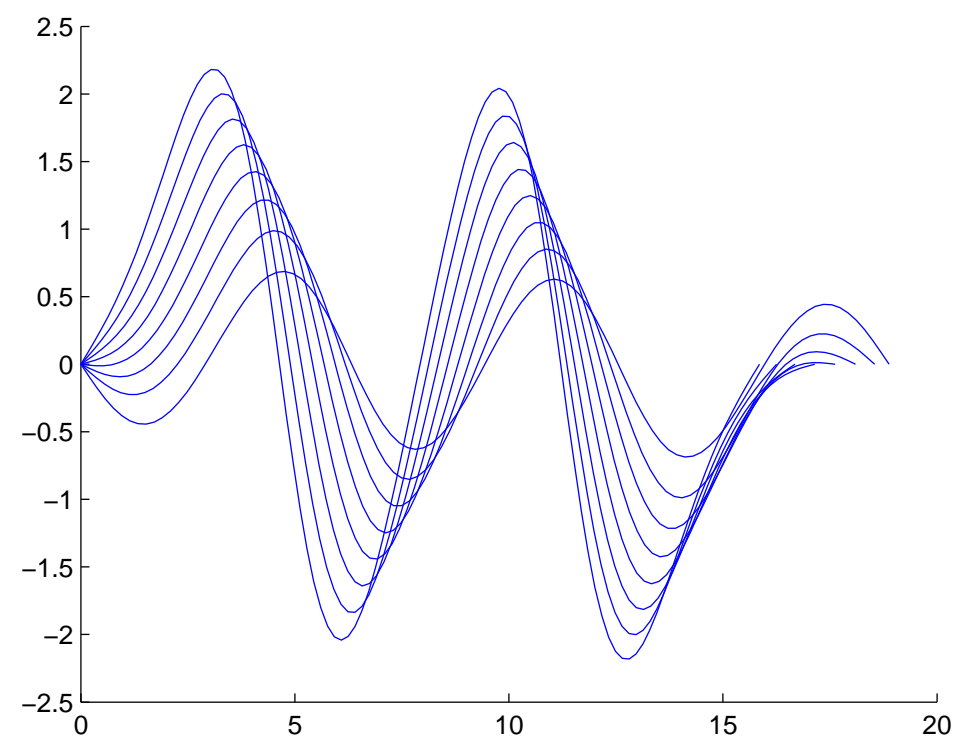

Figura 3.2: Curva de soluções obtidas ao variar o parâmetro $\lambda$ na equação (3.1). 


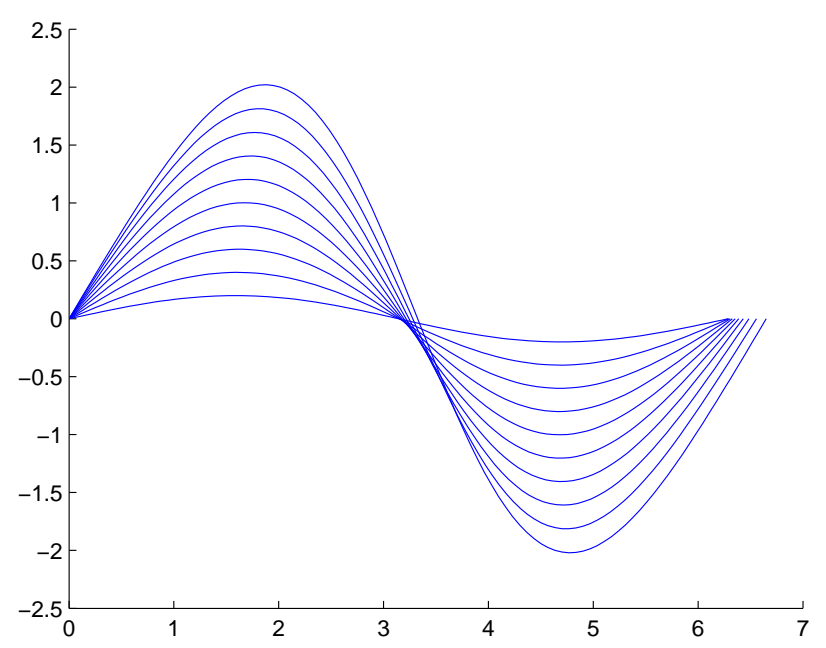

Figura 3.3: Curva de soluções da equação de Michelson obtida ao variar o parâmetro $\lambda$ na equação (3.1).

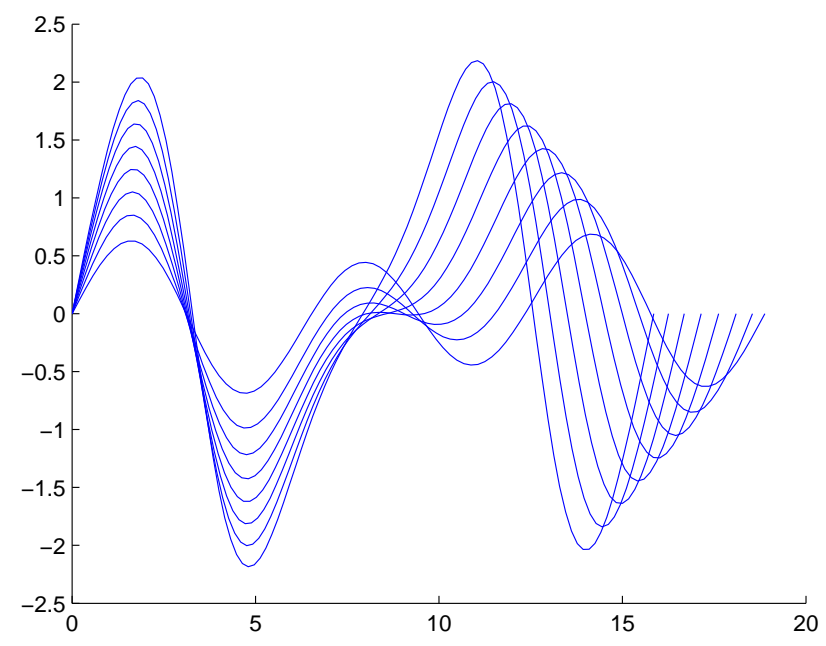

Figura 3.4: Curva de soluções da equação de Michelson obtida ao variar o parâmetro $\lambda$ na equação (3.1). 


\section{Capítulo 4}

\section{Soluções Periódicas do Sistema de Lorenz}

Nesse capítulo provaremos a existência de órbitas periódicas para o seguinte sistema não linear de dimensão três:

$$
\left\{\begin{array}{l}
\dot{x}_{1}=\sigma\left(x_{2}-x_{1}\right) \\
\dot{x}_{2}=\rho x_{1}-x_{2}-x_{1} x_{3} \\
\dot{x}_{3}=x_{1} x_{2}-\beta x_{3}
\end{array}\right.
$$

ou, equivalentemente,

$$
\dot{x}=\underbrace{\left(\begin{array}{ccc}
-\sigma & \sigma & 0 \\
\rho & -1 & 0 \\
0 & 0 & -\beta
\end{array}\right)}_{L(\sigma, \rho, \beta)} x+\left(\begin{array}{c}
0 \\
-x_{1} x_{3} \\
x_{1} x_{2}
\end{array}\right) .
$$

Observe que se $\gamma(t)=\left(\begin{array}{c}\gamma_{1}(t) \\ \gamma_{2}(t) \\ \gamma_{3}(t)\end{array}\right)$ é uma solução periódica de (4.1) então $\gamma(t)$ é de classe $C^{\infty}$. Logo, pelo Corolário 3.2.1 podemos concluir que $\gamma$ pode ser dada em termos de sua série de fourier

$$
\gamma(t)=\sum_{k \in \mathbb{Z}} c_{k} e^{-i k l t}, \quad t \in \mathbb{R}
$$

onde

$$
\begin{gathered}
c_{-k}=\bar{c}_{k}, \quad \forall k \geq 0, \\
l \in \mathbb{R}, l>0, \\
c_{0}=\left(a_{0}^{1}, a_{0}^{2}, a_{0}^{3}\right) \in \mathbb{R}^{3}
\end{gathered}
$$

e

$$
c_{k}=\left(c_{k}^{1}, c_{k}^{2}, c_{k}^{3}\right)=\left(a_{k}^{1}+i b_{k}^{1}, a_{k}^{2}+i b_{k}^{2}, a_{k}^{3}+i b_{k}^{3}\right) \in \mathbb{C}^{3} .
$$

Além disso, pelo Corolário 3.2.1, a sequência

$$
c:=\left(l, a_{0}^{1}, a_{0}^{2}, a_{0}^{3}, a_{1}^{1}, b_{1}^{1}, a_{1}^{2}, b_{1}^{2}, a_{1}^{3}, b_{1}^{3}, a_{2}^{1}, b_{2}^{1}, a_{2}^{2}, b_{2}^{2}, \ldots\right) \in \Omega^{s} \forall s>0
$$

com

$$
\|c\|_{s}=\max \left(\left\|\left(l, a_{0}^{1}, a_{0}^{2}, a_{0}^{3}\right)\right\|_{\infty}, \sup _{k \geq 1}\left\{\left\|\left(a_{k}^{1}, b_{k}^{1}, a_{k}^{2}, b_{k}^{2}, a_{k}^{3}, b_{k}^{3}\right)\right\|_{\infty} \cdot k^{s}\right\}\right)<\infty
$$


Pelo Teorema 3.2.1 podemos escrever

$$
\gamma^{\prime}(t)=\sum_{k \in \mathbb{Z}}-i k l c_{k} e^{-i k l t}
$$

Pelo Teorema 3.2.4 temos

$$
\gamma_{1}(t) \gamma_{3}(t)=\sum_{k \in \mathbb{Z}}\left[\sum_{k_{1}+k_{2}=k} c_{k_{1}}^{1} c_{k_{2}}^{3}\right] e^{-i k l t}=\sum_{k \in \mathbb{Z}}\left(c^{1} * c^{3}\right)_{k} \cdot e^{-i k l t}
$$

e

$$
\gamma_{1}(t) \gamma_{2}(t)=\sum_{k \in \mathbb{Z}}\left[\sum_{k_{1}+k_{2}=k} c_{k_{1}}^{1} c_{k_{2}}^{2}\right] e^{-i k l t}=\sum_{k \in \mathbb{Z}}\left(c^{1} * c^{2}\right)_{k} \cdot e^{-i k l t}
$$

Como $\gamma(t)$ é solução de (4.1), podemos substituir as expressões (4.2), (4.3) e (4.4) no sistema de Lorenz, obtendo-se

$$
\begin{gathered}
\sum_{k \in \mathbb{Z}}-i k l c_{k} e^{-i k l t}=L(\sigma, \rho, \beta) \sum_{k \in \mathbb{Z}} c_{k} e^{-i k l t}+\sum_{k \in \mathbb{Z}}\left[\begin{array}{c}
0 \\
-\left(c^{1} * c^{3}\right)_{k} \\
\left(c^{1} * c^{2}\right)_{k}
\end{array}\right] e^{-i k l t} \Rightarrow \\
\Rightarrow \sum_{k \in \mathbb{Z}}\left[-i k l c_{k}-L(\sigma, \rho, \beta) c_{k}-\left[\begin{array}{c}
0 \\
-\left(c^{1} * c^{3}\right)_{k} \\
\left(c^{1} * c^{2}\right)_{k}
\end{array}\right]\right] \cdot e^{-i k l t}=0 \Rightarrow \\
\Rightarrow-i k l c_{k}-L(\sigma, \rho, \beta) c_{k}-\left[\begin{array}{c}
0 \\
-\left(c^{1} * c^{3}\right)_{k} \\
\left(c^{1} * c^{2}\right)_{k}
\end{array}\right]=0 \quad \forall k \in \mathbb{Z}
\end{gathered}
$$

onde a última implicação deve-se à unicidade dos coeficientes de Fourier. A seguir, defina:

$$
\begin{gathered}
f_{k}: \Omega^{s} \rightarrow \mathbb{C}^{3} \\
f_{k}(c)=-i k l c_{k}-L(\sigma, \rho, \beta) c_{k}-\left[\begin{array}{c}
0 \\
-\left(c^{1} * c^{3}\right)_{k} \\
\left(c^{1} * c^{2}\right)_{k}
\end{array}\right] .
\end{gathered}
$$

Pelas contas acima, fixado $s>2$, concluímos que, se $\gamma(t)=\sum_{k \in \mathbb{Z}} c_{k} e^{-i k l t}$ é solução periódica de (4.1), então $f_{k}(c)=0 \forall k \in \mathbb{Z}$.

Reciprocamente, suponha que existam $s>2$ e

$$
c:=\left(l, a_{0}^{1}, a_{0}^{2}, a_{0}^{3}, a_{1}^{1}, b_{1}^{1}, a_{1}^{2}, b_{1}^{2}, a_{1}^{3}, b_{1}^{3}, a_{2}^{1}, b_{2}^{1}, a_{2}^{2}, b_{2}^{2}, \ldots\right) \in \Omega^{s}
$$

satisfazendo

$$
f_{k}(c)=0, \forall k \geq 0 .
$$

Analogamente ao que foi visto no capítulo anterior, podemos concluir que

$$
f_{-k}(x)=\overline{f_{k}(x)}, \forall k \geq 0
$$

Logo,

$$
f_{k}(c)=0 \quad \forall k \geq 0 \Rightarrow f_{k}(c)=0 \quad \forall k \in \mathbb{Z}
$$

Donde segue que 


$$
\sum_{k \in \mathbb{Z}} f_{k}(c) e^{-i k l t}=\sum_{k \in \mathbb{Z}}\left[-i k l c_{k}-L(\sigma, \rho, \beta) c_{k}-\left[\begin{array}{c}
0 \\
-\left(c^{1} * c^{3}\right)_{k} \\
\left(c^{1} * c^{2}\right)_{k}
\end{array}\right]\right] \cdot e^{-i k l t}=0
$$

Por outro lado, temos que

$$
\left\|-i k l c_{k} e^{-i k l t}\right\|_{\infty}=k l\left\|c_{k}\right\|_{\infty} \leq \frac{l\|c\|_{s}}{k^{s-1}}, \forall t \in \mathbb{R}
$$

Como $s>2$, segue que

$$
\sum_{k \in \mathbb{Z}} \frac{l\|c\|_{s}}{k^{s-1}}<\infty
$$

e, consequentemente, a série $\sum_{k \in \mathbb{Z}}-i k l c_{k} e^{-i k l t}$ converge uniformemente para $\gamma^{\prime}(t)$ em $\mathbb{R}$. Temos ainda que $\sum_{k \in \mathbb{Z}} c_{k} e^{-i k l t}$ converge uniformemente para $\gamma(t)$ em $\mathbb{R}$. De fato,

$$
\left\|c_{k} e^{-i k l t}\right\|_{\infty} \leq \frac{\|c\|_{s}}{k^{s}}, \forall k \geq 1, \forall t \in \mathbb{R}
$$

e a convergência segue pelo critério de Weierstrass. Dadas essas convergências e as equações (4.3) e (4.4), segue que (4.6) pode ser reescrita como

$$
\gamma^{\prime}(t)-L(\sigma, \rho, \beta) \gamma(t)-\left[\begin{array}{c}
0 \\
-\gamma_{1}(t) \gamma_{3}(t) \\
\gamma_{1}(t) \gamma_{2}(t)
\end{array}\right]=0
$$

Donde segue imediatamente que $\gamma(t)$ é solução periódica de (4.1). Com isso, acabamos de provar o seguinte teorema:

Teorema 4.0.2. Sejam $s>2$ fixado e $f_{k}, k \geq 0$, como definidas em (4.5). O sistema (4.1) possui solução periódica $\gamma(t)=\sum_{k \in \mathbb{Z}} c_{k} e^{-i k l t}$ se, e somente se, $f_{k}(c)=0, \forall k \geq 0$, onde $c=\left(l, c_{0}, c_{1}, c_{2}, \ldots\right) \in \Omega^{s}$ com $l \in \mathbb{R}, \quad c_{0} \in \mathbb{R}^{3}, c_{k} \in \mathbb{C}^{3}, k \geq 1$.

Observação 4.0.1. Pelo Corolário 3.2.1 se $c=\left(l, c_{0}, c_{1}, c_{2}, \ldots\right) \in \Omega^{s}$ satisfaz o teorema acima para algum $s>2$ fixado, então $c \in \Omega^{\tilde{s}}$ para todo $\tilde{s}>2$.

O seguinte teorema é a formulação em termos de funções reais do Teorema 4.0.1. Ele é claramente menos consiso em termos de notação e nada acrescenta à versão acima do ponto de vista teórico. Porém, a formulação seguinte é mais simples do ponto de vista prático, pois nos permite trabalhar apenas com funções diferenciáveis. Isto não ocorre no tratamento complexo, uma vez que nesse caso devem surgir termos do tipo $\bar{c}_{k}$. E sabemos que a conjugação complexa não é diferenciável.

Teorema 4.0.3. Seja $s>2$ fixado. Estipule a seguinte notação em $\Omega^{s}$ :

$$
c=\left(l, a_{0}^{1}, a_{0}^{2}, a_{0}^{3}, a_{1}^{1}, a_{1}^{2}, b_{1}^{1}, b_{1}^{2}, b_{1}^{3}, \ldots\right) \in \Omega^{s}
$$

com

$$
c_{0}=\left(\begin{array}{c}
a_{0}^{1} \\
a_{0}^{2} \\
a_{0}^{3}
\end{array}\right) \in \mathbb{R}^{3}, l \in \mathbb{R}, c_{k}=\left(\begin{array}{c}
a_{k}^{1}+i b_{k}^{1} \\
a_{k}^{2}+i b_{k}^{2} \\
a_{k}^{3}+i b_{k}^{3}
\end{array}\right) \in \mathbb{C}^{3}, \forall k \geq 1 .
$$


Considere $\psi_{k}: \Omega^{s} \rightarrow \mathbb{C}^{3}, k \geq 0$, dadas por

$$
\psi_{k}(c)=\left(\begin{array}{c}
\psi_{k, 1}(c) \\
\psi_{k, 2}(c) \\
\psi_{k, 2}(c)
\end{array}\right)=i k l c_{k}+L(\sigma, \rho, \beta) c_{k}+\left[\begin{array}{c}
0 \\
-\left(c^{1} * c^{3}\right)_{k} \\
\left(c^{1} * c^{2}\right)_{k}
\end{array}\right], k \geq 0 .
$$

Para cada $k \geq 0$ defina $f_{k}: \Omega^{s} \rightarrow \mathbb{R}^{6}$ dada por

$$
f_{k}(c)=\left(\begin{array}{c}
\operatorname{Re}\left\{\psi_{k, 1}(c)\right\} \\
\operatorname{Im}\left\{\psi_{k, 1}(c)\right\} \\
\operatorname{Re}\left\{\psi_{k, 2}(c)\right\} \\
\operatorname{Im}\left\{\psi_{k, 2}(c)\right\} \\
\operatorname{Re}\left\{\psi_{k, 3}(c)\right\} \\
\operatorname{Im}\left\{\psi_{k, 3}(c)\right\}
\end{array}\right), k \geq 1
$$

Nessas condições, o sistema (4.1) possui solução periódica $\gamma(t)=\sum_{k \in \mathbb{Z}} c_{k} e^{-i k l t}$ se, $e$ somente se,

$$
f_{k}(c)=0, \forall k \geq 0
$$

Observação 4.0.2. Note que, pelo Corolário 3.1.2, para cada $c \in \Omega^{s}$, podemos concluir que $\left\{f_{k}(c)\right\}_{k=0}^{\infty} \in \Omega^{s-1}$. Logo, o Teorema 4.0.3 afirma que, para provarmos a existência de órbitas periódicas do sistema (4.1), é suficiente provarmos a existência de raízes para a função

$$
\begin{aligned}
f: & \Omega^{s} \rightarrow \Omega^{s-1} \\
c & \longmapsto\left\{f_{k}(c)\right\}_{k \geq 0}
\end{aligned}
$$

para algum $s>2$, onde estamos considerando $f_{k}: \Omega^{s} \rightarrow \mathbb{R}^{6}$ como dadas no enunciado do Teorema 4.0.3.

Os métodos de ponto fixo que estamos interessados em utilizar identificam apenas raízes isoladas. Porém, sabemos do capítulo anterior que uma mesma órbita periódica está associada a diferentes raízes de $f$,dada por (4.7), que estão arbitrariamente próximas entre si. A seguir, estabelecemos uma restrição sobre as condições iniciais das soluções que parametrizam uma mesma órbita de modo que as soluções que satisfazem a esta restrição ficam naturalmente isoladas entre si. Mais precisamente, buscamos apenas por soluções que parametrizam uma mesma órbita e que satisfaçam condições iniciais que se encontram sobre uma região do espaço de fase em que a órbita em questão possua um número finito de pontos em comum.

\subsection{Condição de Fase de Poincaré}

O não isolamento das raízes da função $f$ dada em (4.7) se deve às múltilas parametrizações possíveis de uma mesma órbita periódica de um campo autônomo. Cada uma das parametrizações é proveniente de uma condição inicial diferente sobre a órbita. Se $\gamma(t)$ é uma solução que parametriza uma órbita $\Gamma$, então, para cada $\tau \in \mathbb{R}$, a função $\gamma_{\tau}(t):=\gamma(t+\tau)$ também é uma solução que parametriza a mesma órbita $\Gamma$. Além disso, estas são as únicas soluções que parametrizam a órbita $\Gamma$. A fim de isolar uma das outras soluções que parametrizam uma mesma órbita, adiciona-se uma condição de fase ao problema. No capítulo anterior esta condição consistia em impor que algum coeficiente de Fourier da solução tivesse parte real nula. Além disso, no caso da equação de 
Michelson, não foi necessário conhecer previamente uma solução aproximada para que houvesse convergência do Método de Newton. Ou seja, usando chutes iniciais aleatórios, o método convergiu, fornecendo soluções muito próximas das verdadeiras. No caso das equações de Lorenz, usando a mesma condição de fase dada pelo capítulo anterior, chutes iniciais aleatórios não foram suficientes para que ocorresse convergência. Mesmo usando condições iniciais dadas por soluções periódicas obtidas numericamente, o método não convergiu para soluções não nulas. Sendo assim, foi necessário mudar a condição de fase do problema. Optamos pela condição de fase de Poincaré que descrevemos na sequência. Veremos que essa condição só faz sentido se conhecermos previamente alguma solução periódica proveniente de algum método numérico. Sendo assim, o método que estamos descrevendo neste trabalho é útil em provar a validade do método numérico que forneceu determinada órbita, bem como fornecer um meio de continuação de soluções com relação a parâmetos, como veremos no final deste capítulo.

Suponha conhecida alguma solução periódica aproximada obtida por meio de algum método numérico apropriado (no nosso caso, obtemos tal solução pelo diagrama de bifurcação fornecido pelo pacote MatCont [3]). Denotemos por $\gamma_{0}(t)$ a solução assim obtida, por $\Gamma_{0}$ a órbita correspondente e por $\Sigma$ o plano que passa por $\gamma_{0}(0)$ e que é perpendicular a $\gamma_{0}^{\prime}(0)$. Podemos supor que $\Gamma$ está próxima de $\Gamma_{0}$ no espaço de fase e, portanto, também intercepta o plano $\Sigma$.

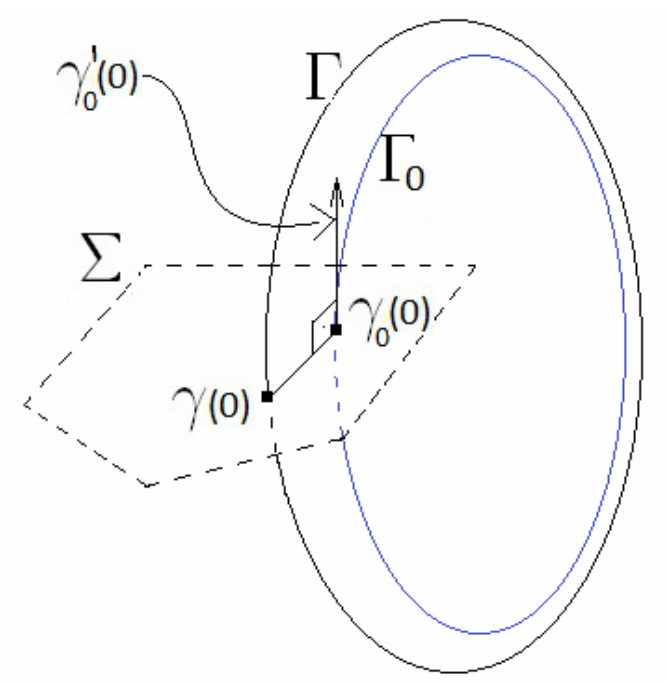

Figura 4.1: Isolamos as soluções que prametrizam a órbita $\Gamma$ impondo que a condição inicial esteja sobre o plano $\Sigma$.

Impondo a condição $\gamma(0) \in \Sigma$, que pode ser equacionada por

$$
\left\langle\gamma(0)-\gamma_{0}(0), \gamma_{0}^{\prime}(0)\right\rangle=0
$$

restringimos nossa busca apenas às soluções $\gamma(t)$ que possuem ponto inicial $\gamma(0)$ sobre o plano $\Sigma$. Note que $\Gamma$ cruza $\Sigma$ num número finito de pontos. Ou seja, apenas para finitos valores de $\tau$, as parametrizações $\gamma_{\tau}(t)$ satisfazem à condição acima, pois a cada $\tau$ está associada uma única condição inicial.

Da discussão acima podemos concluir que, se $f$ é como em (4.7) e existem soluções para o problema

$$
\left\{\begin{array}{l}
\left\langle\gamma(0)-\gamma_{0}(0), \gamma_{0}^{\prime}(0)\right\rangle=0 \\
f(c)=0
\end{array}\right.
$$


então estas soluções estão isoladas entre si. Sendo assim, deixamos de buscar por raízes de $f$ dada por (4.7) e passamos a buscar por raízes da função

$$
F(c)=\left\{\eta(c), f_{0}(c), f_{1}(c), \ldots\right\}
$$

onde

$$
\eta(c)=\left\langle\gamma(0)-\gamma_{0}(0), \gamma_{0}^{\prime}(0)\right\rangle
$$

está expressa em (4.9).

Denotemos por

$$
\gamma_{0}(t)=\sum_{k \in \mathbb{Z}}\left(U_{k}+i V_{k}\right) e^{-i k l_{0} t}
$$

onde

$$
U_{k}=V_{k}=0, \forall k \geq m
$$

e

$$
c=\left\{l, a_{0}^{1}, a_{0}^{2}, a_{0}^{3}, a_{1}^{1}, b_{1}^{1}, a_{1}^{2}, b_{1}^{2}, \ldots\right\} .
$$

Lembre que $\gamma_{0}(t)$ é uma solução aproximada obtida em simulação numérica. Nos limitamos a manipular um número finito $m$ de coeficientes de Fourier desta solução igual ao número de coeficientes que estamos levando em consideração no Método de Newton. Nesses termos, temos:

$$
\begin{aligned}
& \eta(c)=\left\langle\left(\begin{array}{c}
a_{0}^{1} \\
a_{0}^{2} \\
a_{0}^{3}
\end{array}\right)-\left(\begin{array}{c}
U_{0}^{1} \\
U_{0}^{2} \\
U_{0}^{3}
\end{array}\right)+2 \sum_{k=1}^{\infty} a_{k}-U_{k}, \sum_{k=1}^{\infty} k V_{k}\right\rangle, \\
& f_{0}(c)=L(\sigma, \rho, \beta) a_{0}+\left[\begin{array}{c}
0 \\
-\sum_{k_{1}+k_{2}=0} a_{k_{1}}^{1} a_{k_{2}}^{3}-b_{k_{1}}^{1} b_{k_{2}}^{3} \\
\sum_{k_{1}+k_{2}=0}^{1} a_{k_{1}}^{1} a_{k_{2}}^{2}-b_{k_{1}}^{1} b_{k_{2}}^{2}
\end{array}\right] \\
& f_{k}(c)=\left(\begin{array}{cccccc}
-\sigma & -k l & \sigma & 0 & 0 & 0 \\
k l & -\sigma & 0 & \sigma & 0 & 0 \\
\rho & 0 & -1 & -k l & 0 & 0 \\
0 & \rho & k l & -1 & 0 & 0 \\
0 & 0 & 0 & -\beta & -k l & 0 \\
0 & 0 & 0 & 0 & k l & -\beta
\end{array}\right) c_{k}+\left\{\begin{array}{c}
0 \\
0 \\
-\sum_{k_{1}+k_{2}=k} a_{k_{1}}^{1} a_{k_{2}}^{3}-b_{k_{1}}^{1} b_{k_{2}}^{3} \\
-\sum_{k_{1}+k_{2}=k}^{1} a_{k_{1}}^{1} b_{k_{2}}^{3}+b_{k_{1}}^{1} a_{k_{2}}^{3} \\
\sum_{k_{1}+k_{2}=k} a_{k_{1}}^{1} a_{k_{2}}^{2}-b_{k_{1}}^{1} b_{k_{2}}^{2} \\
\sum_{k_{1}+k_{2}=k}^{2} a_{k_{1}}^{1} b_{k_{2}}^{2}+b_{k_{1}}^{1} a_{k_{2}}^{2}
\end{array}\right\}, k \geq 1
\end{aligned}
$$

Escolhendo $m=20$ e aplicando o Método de Newton à projeção finita de ordem $m$ da função $F$, dada por

$$
F^{m}\left(l, c_{0}, \ldots, c_{m-1}, 0_{\infty}\right)=\left(\begin{array}{c}
\eta\left(l, c_{0}, \ldots, c_{m-1}, 0_{\infty}\right) \\
f_{0}\left(l, c_{0}, \ldots, c_{m-1}, 0_{\infty}\right) \\
\vdots \\
f_{m-1}\left(l, c_{0}, \ldots, c_{m-1}, 0_{\infty}\right)
\end{array}\right)
$$

obtemos uma raiz aproximada de $F$, que denotaremos por $\left(\bar{c}, 0_{\infty}\right)$ com $\bar{c} \in \mathbb{R}^{6 m-2}$. 
Nossa meta nas seções seguintes é descrever um método computacional rigoroso para provar a existência de uma raiz de $F$ numa vizinhança de $\left(\bar{c}, 0_{\infty}\right)$. Como raízes de $F$ correspondem a soluções periódicas do sistema de Lorenz, teremos concluído o objetivo deste capítulo.

\subsection{Polinômios radiais para o sistema de Lorenz}

O método dos polinômios radiais está intrinsecamente ligado à verificação das hipóteses do seguinte teorema, as quais dão condições suficientes para a existência raízes da $F$ dada em (4.8), e de órbitas periódicas para o sistema de Lorenz.

Teorema 4.2.1. Sejam $T(x)=x-A F(x), x \in \Omega^{s}$ onde F é como em (4.8) e $A: \Omega^{s-1} \rightarrow$ $\Omega^{s}$ é uma aplicação linear inversivel. Suponha que existam $\bar{x}, Z(r)$ e $Y$ em $\Omega^{s}$ tais que

$$
\begin{gathered}
\sup _{u, v \in B(0,1)}\left|(D T(\bar{x}+r u) \cdot r v)_{k}\right| \leq_{c . c} Z_{k}(r), \forall k \geq 0 \\
\left|(T(\bar{x})-\bar{x})_{k}\right| \leq_{c . c} Y_{k} \forall k \geq 0 .
\end{gathered}
$$

Nessas condições, se existir $r>0$ tal que

$$
\|Z(r)+Y\|_{s}<r \Leftrightarrow p_{k, i}(r)=Z_{k, i}(r)+Y_{k, i}-\frac{r}{\omega_{k}^{s}}<0, k \geq 0, i \in\{1, \ldots, 6\},
$$

então $T$ possui um único ponto fixo $\hat{x}$ na bola $B(\bar{x}, r)$. Em particular, $F(\hat{x})=0$ e (4.1) possui uma órbita periódica.

A demonstração deste teorema é idêntica à demonstração do Teorema 3.6.1.

O mesmo argumento heurístico apresentado para a construção do operador $A$ no caso da equação de Michelson pode ser aplicado aqui. Logo, no Teorema 4.2.1 tomaremos

$$
A=\left(\begin{array}{cccc}
A^{m} & & & \\
& \Lambda_{m}^{-1}(\bar{x}) & & \\
& & \Lambda_{m+1}^{-1}(\bar{x}) & \\
& & & \ddots
\end{array}\right)
$$

onde $A^{m}$ é uma inversa numérica da jacobiana de $F^{m}$ num ponto $\bar{c}$ tal que $F^{m}(\bar{c}) \approx 0$ e $\Lambda_{k}(\bar{x}), \bar{x}=\left\{\bar{c}, 0_{\infty}\right\}, k \geq m$, são as derivadas parciais $\frac{\partial F_{k}}{x_{k}}(\bar{x})$ expressas abaixo, em (4.15).

Para $k \geq m$ temos:

$$
\Lambda_{k}(\bar{x}):=\frac{\partial F_{k}}{\partial x_{k}}(\bar{x})=\left(\begin{array}{cccccc}
-\sigma & -k \bar{l} & \sigma & 0 & 0 & 0 \\
k \bar{l} & -\sigma & 0 & \sigma & 0 & 0 \\
\rho-\bar{a}_{0}^{3} & 0 & -1 & -k \bar{l} & -\bar{a}_{0}^{1} & 0 \\
0 & \rho-\bar{a}_{0}^{3} & k \bar{l} & -1 & 0 & -\bar{a}_{0}^{1} \\
\bar{a}_{0}^{2} & 0 & \bar{a}_{0}^{1} & 0 & -\beta & -k \bar{l} \\
0 & \bar{a}_{0}^{2} & 0 & \bar{a}_{0}^{1} & k \bar{l} & -\beta
\end{array}\right) .
$$

Mostremos a seguir que existe $m>0$ tal que $\Lambda_{k}(\bar{x})$ é inversível para todo $k \geq m$.

Definição 4.2.1. Seja $M=\left(m_{i j}\right)$ uma matriz real quadrada de ordem $n$. Dizemos que $M$ é estritamente diagonalmente dominante se $\left|m_{i i}\right|>\sum_{j \neq i}\left|m_{i j}\right|, \quad \forall i \in\{1,2, \ldots, n\}$ 
Teorema 4.2.2. Seja $M=\left(m_{i j}\right)$ uma matriz real quadrada de ordem $n$. Se $M$ é estritamente diagonalmente dominante então $M$ é invertível e $\left\|M^{-1} x\right\|_{\infty} \leq \frac{1}{\alpha}\|x\|_{\infty}, \forall x \in \mathbb{R}^{n}$, onde $\alpha=\min _{1 \leq i \leq n}\left(\left|m_{i i}\right|-\sum_{j \neq i}\left|m_{i j}\right|\right)$.

Para demonstração, ver [4].

Teorema 4.2.3. As matrizes $\Lambda_{k}(\bar{x})$ como definidas em (4.17) são invertiveis sempre que

$$
k \geq \max \left\{\frac{2|\sigma|}{|\bar{l}|}, \frac{\left|\rho-\bar{a}_{0}^{3}\right|+\left|\bar{a}_{0}^{1}\right|+1}{|\bar{l}|}, \frac{|\beta|+\left|\bar{a}_{0}^{1}\right|+\left|\bar{a}_{0}^{2}\right|}{|\bar{l}|}\right\}
$$

e, além disso,

$$
\left\|\Lambda_{k}(\bar{x})^{-1} x\right\|_{\infty} \leq \frac{1}{\alpha}\|x\|_{\infty}, \forall x \in \mathbb{R}^{6}
$$

onde

$$
\alpha=\min \left\{k-\frac{2|\sigma|}{|\bar{l}|}, k-\frac{\left|\rho-\bar{a}_{0}^{3}\right|+\left|\bar{a}_{0}^{1}\right|+1}{|\bar{l}|}, k-\frac{|\beta|+\left|\bar{a}_{0}^{1}\right|+\left|\bar{a}_{0}^{2}\right|}{|\bar{l}|}\right\} .
$$

Demonstração. Temos

$$
\Lambda_{k}(\bar{x})=\left(\begin{array}{cccccc}
\sigma & -\sigma & 0 & k \bar{l} & 0 & 0 \\
\rho & 1 & 0 & 0 & k \bar{l} & 0 \\
0 & 0 & \beta & 0 & 0 & k \bar{l} \\
-k \bar{l} & 0 & 0 & \sigma & -\sigma & 0 \\
0 & -k \bar{l} & 0 & -\rho & 1 & 0 \\
0 & 0 & -k \bar{l} & 0 & 0 & \beta
\end{array}\right)
$$

Permutando as colunas da matriz $\Lambda_{k}(\bar{x})$, obtemos a matriz:

$$
B_{k}(\bar{x})=\left(\begin{array}{cccccc}
-k \bar{l} & -\sigma & 0 & \sigma & 0 & 0 \\
-\sigma & k \bar{l} & \sigma & 0 & 0 & 0 \\
0 & \rho-\bar{a}_{0}^{3} & -k \bar{l} & -1 & 0 & -\bar{a}_{0}^{1} \\
\rho-\bar{a}_{0}^{3} & 0 & -1 & k \bar{l} & -\bar{a}_{0}^{1} & 0 \\
0 & \bar{a}_{0}^{2} & 0 & \bar{a}_{0}^{1} & -k \bar{l} & -\beta \\
\bar{a}_{0}^{2} & 0 & \bar{a}_{0}^{1} & 0 & -\beta & k \bar{l}
\end{array}\right)
$$

Observe que se $k \bar{l}>\max \left\{2|\sigma|,\left|\rho-\bar{a}_{0}^{3}\right|+\left|\bar{a}_{0}^{1}\right|+1,\left|\bar{a}_{0}^{1}\right|+\left|\bar{a}_{0}^{2}\right|+|\beta|\right\}$ então $B_{k}(\bar{x})$ é estritamente diagonalmente dominante. Logo, pelo teorema anterior, $B_{k}(\bar{x})$ é intertível e

$$
\left\|B_{k}(\bar{x})^{-1} x\right\|_{\infty} \leq \frac{1}{\mu_{k}}\|x\|_{\infty}, \forall x \in \mathbb{R}^{6}
$$

onde $\mu_{k}=k \bar{l}-\max \left\{2|\sigma|,\left|\rho-\bar{a}_{0}^{3}\right|+\left|\bar{a}_{0}^{1}\right|+1,\left|\bar{a}_{0}^{1}\right|+\left|\bar{a}_{0}^{2}\right|+|\beta|\right\}$.

Por fim, basta observar que $\left\|\Lambda_{k}(\bar{x})\right\|_{\infty}=\left\|B_{k}(\bar{s})\right\|_{\infty}$ de onde segue que $\Lambda_{k}(\bar{x})$ também é inversível e que $\left\|\Lambda_{k}^{-1}(\bar{x})\right\|_{\infty}=\left\|B^{-1}\right\|_{\infty} \leq \frac{1}{\mu_{k}}$.

Na prática, escolhe-se o menor natural $m>0$ tal que $m \bar{l}>\max \left\{2|\sigma|,\left|\rho-\bar{a}_{0}^{3}\right|+\left|\bar{a}_{0}^{1}\right|+\right.$ $\left.1,\left|\bar{a}_{0}^{1}\right|+\left|\bar{a}_{0}^{2}\right|+|\beta|\right\}$. Se a aproximação $\bar{c}$ obtida pelo Método de Newton tiver dimensão $m^{\prime}<m$, substituímos a sequência $\bar{c}$ por $\left(\bar{c}, 0_{6\left(m-m^{\prime}\right)}\right)$, onde $0_{6\left(m-m^{\prime}\right)}$ é o elemento neutro do espaço $\mathbb{R}^{6\left(m-m^{\prime}\right)}$, e seguimos com o cálculo das cotas $Z(r)$ e $Y$ usando essa nova sequência de coeficientes. 
Corolário 4.2.1. Para todo $k \geq m$, onde $m$ é o menor número natural satisfazendo $m \bar{l}>\max \left\{2|\sigma|,\left|\rho-\bar{a}_{0}^{3}\right|+\left|\bar{a}_{0}^{1}\right|+1,\left|\bar{a}_{0}^{1}\right|+\left|\bar{a}_{0}^{2}\right|+|\beta|\right\}$ e para todo $x \in R^{6}$, temos

$$
\left\|\Lambda_{k}(\bar{x})^{-1} x\right\|_{\infty} \leq \frac{1}{k} C_{m}\|x\|_{\infty}
$$

onde

$$
C_{m}=\frac{1}{|\bar{l}|-\frac{1}{m} \max \left\{2|\sigma|,\left|\rho-\bar{a}_{0}^{3}\right|+\left|\bar{a}_{0}^{1}\right|+1,\left|\bar{a}_{0}^{1}\right|+\left|\bar{a}_{0}^{2}\right|+|\beta|\right\}} .
$$

Demonstração. Basta observar que para $k \geq m$ podemos escrever

$$
\begin{gathered}
\mu_{k}=k\left(|\bar{l}|-\frac{1}{k} \max \left\{2|\sigma|,\left|\rho-\bar{a}_{0}^{3}\right|+\left|\bar{a}_{0}^{1}\right|+1,\left|\bar{a}_{0}^{1}\right|+\left|\bar{a}_{0}^{2}\right|+|\beta|\right\}\right) \geq \\
\geq k\left(|\bar{l}|-\frac{1}{m} \max \left\{2|\sigma|,\left|\rho-\bar{a}_{0}^{3}\right|+\left|\bar{a}_{0}^{1}\right|+1,\left|\bar{a}_{0}^{1}\right|+\left|\bar{a}_{0}^{2}\right|+|\beta|\right\}\right), \quad \forall k \geq m .
\end{gathered}
$$

Logo,

$$
\frac{1}{\mu_{k}} \leq \frac{C_{m}}{k}
$$

onde

$$
C_{m}=\frac{1}{|\bar{l}|-\frac{1}{m} \max \left\{2|\sigma|,\left|\rho-\bar{a}_{0}^{3}\right|+\left|\bar{a}_{0}^{1}\right|+1,\left|\bar{a}_{0}^{1}\right|+\left|\bar{a}_{0}^{2}\right|+|\beta|\right\}} .
$$

Assim, para $m$ satisfazendo as hipóteses do corolário acima, podemos definir o operador linear:

$$
A=\left(\begin{array}{cccc}
A^{m} & & & \\
& \Lambda_{m}^{-1}(\bar{x}) & & \\
& & \Lambda_{m+1}^{-1}(\bar{x}) & \\
& & & \ddots
\end{array}\right),
$$

em que $A^{m}$ denota uma inversa numérica da jacobiana $J F^{m}(\bar{c})$.

Dado $s>2$, decorre do Corolário 4.1.1 que

$$
A: \Omega^{s-1} \rightarrow \Omega^{s}
$$

Consequentemente, o operador definido em $\Omega^{s}$ pela expressão

$$
T(x)=x-A F(x)
$$

é tal que

$$
T: \Omega^{s} \rightarrow \Omega^{s}
$$

Agora já estamos em condições de calcular os limitantes $Z(r)$ e $Y$ satisfazendo (4.12) e (4.13). 


\subsubsection{Cálculo de $\mathrm{Z}(\mathrm{r})$}

Sejam $u$ e $v$ pertencentes à bola unitária de $\Omega^{s}$, ou seja, $\left\|u_{k}\right\|_{\infty} \leq \omega_{k}^{-s}$ e $\left\|v_{k}\right\|_{\infty} \leq \omega_{k}^{-s}$ para todo $k \geq 0$. Temos que $D T(\bar{x}+r u) r v=I d \cdot(r v)-A \cdot D F(\bar{x}+r u) \cdot(r v)$.

Dada a estrutura do operador $A$, precisamos estimar separadamente a parte principal, composta pelos primeiros $m$ blocos de componentes de $D T(\bar{x}+r u) r v$, e a parte restante, que chamaremos de calda.

Para estimar a parte principal, escrevemos:

$$
\begin{gathered}
(D T(\bar{x}+r u) \cdot r v)^{m}=r v^{m}-A^{m}\left(\begin{array}{c}
D F_{0}(\bar{x}+r u) \cdot r v \\
\vdots \\
D F_{m-1}(\bar{x}+r u) \cdot r v
\end{array}\right)= \\
=r v^{m}-A^{m}\left(\begin{array}{c}
D F_{0}(\bar{x}) \cdot r v \\
\vdots \\
D F_{m-1}(\bar{x}) \cdot r v
\end{array}\right)-A^{m}\left(\begin{array}{c}
D F_{0}(\bar{x}+r u) \cdot r v-D F_{0}(\bar{x}) \cdot r v \\
\vdots \\
D F_{m-1}(\bar{x}+r u) \cdot r v-D F_{m-1}(\bar{x}) \cdot r v
\end{array}\right) .
\end{gathered}
$$

Além disso, definindo $S^{m}: \mathbb{R}^{6 m-2} \rightarrow \Omega^{s}, S(x)=\left(x, 0_{\infty}\right)$ (a qual é claramente linear), podemos escrever:

$$
\begin{gathered}
F_{k}^{m}(x)=F_{k}\left(x, 0_{\infty}\right)=F_{k} \circ S^{m}(x) \Rightarrow D F_{k}^{m}(\bar{c}) \cdot v^{m}= \\
=D F_{k}\left(\bar{c}, 0_{\infty}\right) \cdot\left(v^{m}, 0_{\infty}\right)=D F_{k}\left(\bar{c}, 0_{\infty}\right) \cdot\left[v-\left(0, \ldots 0, v_{m}, v_{m+1}, v_{m+2}, \ldots\right)\right] \Rightarrow \\
\Rightarrow D F_{k}(\bar{x}) \cdot v=D F_{k}^{m}(\bar{c}) \cdot v^{m}+D F_{k}(\bar{x}) \cdot\left(0, \ldots 0, v_{m}, v_{m+1}, v_{m+2}, \ldots\right) .
\end{gathered}
$$

Substituindo esta identidade na igualdade acima, obtemos

$$
\begin{aligned}
(D T(\bar{x}+r u) \cdot r v)^{m}= & r v^{m}-A^{m} D F^{m}(\bar{c}) \cdot r v^{m}-r A^{m}\left(\begin{array}{c}
D F_{0}(\bar{x}) \cdot\left(0, \ldots 0, v_{m}, v_{m+1}, \ldots\right) \\
\vdots \\
D F_{m-1}(\bar{x}) \cdot\left(0, \ldots 0, v_{m}, v_{m+1}, \ldots\right)
\end{array}\right)- \\
& -A^{m}\left(\begin{array}{c}
D F_{0}(\bar{x}+r u) \cdot r v-D F_{0}(\bar{x}) \cdot r v \\
\vdots \\
D F_{m-1}(\bar{x}+r u) \cdot r v-D F_{m-1}(\bar{x}) \cdot r v
\end{array}\right)
\end{aligned}
$$

Logo,

$$
\begin{aligned}
\left|(D T(\bar{x}+r u) \cdot r v)^{m}\right| & \leq_{c . c}\left|I d-A^{m} D F^{m}(\bar{c})\right|\left|v^{m}\right| r+\left|A^{m}\right|\left|\left(\begin{array}{c}
D F_{0}(\bar{x}) \cdot\left(0, \ldots 0, v_{m}, v_{m+1}, \ldots\right) \\
\vdots \\
D F_{m-1}(\bar{x}) \cdot\left(0, \ldots 0, v_{m}, v_{m+1}, \ldots\right)
\end{array}\right)\right| r+ \\
& +\left|A^{m}\right|\left|\left(\begin{array}{c}
D F_{0}(\bar{x}+r u) \cdot r v-D F_{0}(\bar{x}) \cdot r v \\
\vdots \\
D F_{m-1}(\bar{x}+r u) \cdot r v-D F_{m-1}(\bar{x}) \cdot r v
\end{array}\right)\right|
\end{aligned}
$$

A fim de estimar as duas últimas parcelas do lado direito de (4.17), precisamos conhecer as expressões das derivadas $D F_{k}(x) v$, onde $x, v \in \Omega^{s}$. Para isso, adote a seguinte notação para $x$ e $v$ :

$$
x=\left(l, a_{0}^{1}, a_{0}^{2}, a_{0}^{3}, a_{1}^{1}, b_{1}^{1}, a_{1}^{2}, b_{1}^{2}, a_{1}^{3}, b_{1}^{3}, \ldots\right),
$$




$$
v=\left(v_{00}, v_{0,1}, v_{0,2}, v_{0,3}, v_{1,1}^{1}, v_{1,2}^{1}, v_{1,1}^{2}, v_{1,2}^{2}, v_{1,1}^{3}, v_{1,2}^{3}, \ldots\right) .
$$

Segundo a notação acima, temos:

$$
D \eta(x) v=\left\langle\left(E_{1}, E_{2}, E_{3}\right), v_{0}\right\rangle+2\left\langle\left(E_{1}, 0, E_{2}, 0, E_{3}, 0\right), \sum_{j=1}^{\infty} v_{j}\right\rangle
$$

onde

$$
E_{1}=\sum_{k=1}^{m-1} k V_{k}^{1}, E_{2}=\sum_{k=1}^{m-1} k V_{k}^{2}, E_{3}=\sum_{k=1}^{m-1} k V_{k}^{3}
$$

$\mathrm{e}$

$$
D f_{0}(x) v=L(\sigma, \rho, \beta) v_{00}+\left(\begin{array}{c}
0 \\
-\sum_{k_{1}+k_{2}=0} a_{k_{1}}^{1} v_{k_{2}, 1}^{3}+a_{k_{2}}^{3} v_{k_{1}, 1}^{1}-b_{k_{1}}^{1} v_{k_{2}, 2}^{3}-b_{k_{2}}^{3} v_{k_{1}, 2}^{1} \\
\sum_{k_{1}+k_{2}=0} a_{k_{1}}^{1} v_{k_{2}, 1}^{2}+a_{k_{2}}^{2} v_{k_{1}, 1}^{1}-b_{k_{1}}^{1} v_{k_{2}, 2}^{2}-b_{k_{2}}^{2} v_{k_{1}, 2}^{1}
\end{array}\right), k \geq 1
$$

$$
\begin{gathered}
D f_{k}(x) v=\left(\begin{array}{c}
-k b_{k}^{1} \\
k a_{k}^{1} \\
-k b_{k}^{2} \\
k a_{k}^{2} \\
-k b_{k}^{3} \\
k a_{k}^{3}
\end{array}\right) v_{00}+\left(\begin{array}{cccccc}
-\sigma & -k l & \sigma & 0 & 0 & 0 \\
k l & -\sigma & 0 & \sigma & 0 & 0 \\
\rho & 0 & -1 & -k l & 0 & 0 \\
0 & \rho & k l & -1 & 0 & 0 \\
0 & 0 & 0 & 0 & -\beta & -k l \\
0 & 0 & 0 & 0 & k l & -\beta
\end{array}\right) v_{k}+ \\
0 \\
0 \\
+\left(\begin{array}{c}
-\sum_{k_{1}+k_{2}=k} a_{k_{1}}^{1} v_{k_{2}, 1}^{3}+a_{k_{2}}^{3} v_{k_{1}, 1}^{1}-b_{k_{1}}^{1} v_{k_{2}, 2}^{3}-b_{k_{2}}^{3} v_{k_{1}, 2}^{1} \\
-\sum_{k_{1}+k_{2}=k} a_{k_{1}}^{1} v_{k_{2}, 2}^{3}+b_{k_{2}}^{3} v_{k_{1}, 1}^{1}+a_{k_{2}}^{3} v_{k_{1}, 2}^{1}+b_{k_{1}}^{1} v_{k_{2}, 1}^{3} \\
\sum_{k_{1}+k_{2}=k} a_{k_{1}}^{1} v_{k_{2}, 1}^{2}+a_{k_{2}}^{2} v_{k_{1}, 1}^{1}-b_{k_{1}}^{1} v_{k_{2}, 2}^{2}-b_{k_{2}}^{2} v_{k_{1}, 2}^{1} \\
\sum_{k_{1}+k_{2}=k}^{1} a_{k_{1}}^{1} v_{k_{2}, 2}^{2}+b_{k_{2}}^{2} v_{k_{1}, 1}^{1}+a_{k_{2}}^{2} v_{k_{1}, 2}^{1}+b_{k_{1}}^{1} v_{k_{2}, 1}^{2}
\end{array}\right), k \geq 1 .
\end{gathered}
$$

Podemos usar as fórmulas acima, observando que o termo envolvendo somatórios é linear em $x$ e o fato de que $\left\|u_{k}\right\|_{\infty} \leq \omega_{k}^{-s}$ e $\left\|v_{k}\right\|_{\infty} \leq \omega_{k}^{-s}$, para obter a seguinte estimativa para as diferenças $\left|D F_{k}(\bar{x}+r u) r v-D F_{k}(\bar{x}) r v\right|$ :

$$
\begin{gathered}
\left|D f_{0}(\bar{x}+r u) r v-D f_{0}(\bar{x}) r v\right| \leq_{c . c .} \underbrace{\left[8 \sigma(s)\left(\begin{array}{l}
0 \\
0 \\
1 \\
1
\end{array}\right)+\left(\begin{array}{l}
0 \\
0 \\
2 \\
2
\end{array}\right)\right]}_{\eta_{0}} r^{2} \\
\left|D f_{k}(\bar{x}+r u) r v-D f_{k}(\bar{x}) r v\right| \leq_{c . c .} \underbrace{\left[\frac{8 \sigma_{k}(s)}{k^{s}}\left(\begin{array}{l}
0 \\
0 \\
1 \\
1 \\
1 \\
1
\end{array}\right)+\frac{2}{k^{s-1}}\left(\begin{array}{l}
1 \\
1 \\
1 \\
1 \\
1 \\
1
\end{array}\right)\right]}_{\eta_{k}} r^{2}, k \geq 1 .
\end{gathered}
$$


Defina

$$
E=\left(\begin{array}{c}
\eta_{0} \\
\eta_{1} \\
\vdots \\
\eta_{m-1}
\end{array}\right)
$$

Quanto aos termos da forma $D f_{k}(\bar{x})\left(0, \ldots, 0, v_{m}, v_{m+1}, \ldots\right)$, começamos lembrando que:

$$
D f_{k}(\bar{x})\left(0, \ldots, 0, v_{m}, v_{m+1}, \ldots\right)=\sum_{j=m}^{\infty} \frac{\partial F_{k}}{\partial x_{j}}(\bar{x}) v_{j} .
$$

Ainda, para $1 \leq k \leq m-1$ e $j \geq m$, temos:

$$
\frac{\partial f_{k}}{\partial x_{j}}(\bar{x})=\left(\begin{array}{cccccc}
0 & 0 & 0 & 0 & 0 & 0 \\
0 & 0 & 0 & 0 & 0 & 0 \\
-\bar{a}_{j-k}^{3} & -\bar{b}_{j-k}^{3} & 0 & 0 & -\bar{a}_{j-k}^{1} & -\bar{b}_{j-k}^{1} \\
\bar{b}_{j-k}^{3} & -\bar{a}_{j-k}^{3} & 0 & 0 & \bar{b}_{j-k}^{1} & -\bar{a}_{j-k}^{1} \\
\bar{a}_{j-k}^{2} & \bar{b}_{j-k}^{2} & \bar{a}_{j-k}^{1} & \bar{b}_{j-k}^{1} & 0 & 0 \\
-\bar{b}_{j-k}^{2} & \bar{a}_{j-k}^{2} & -\bar{b}_{j-k}^{1} & -\bar{a}_{j-k}^{1} & 0 & 0
\end{array}\right)
$$

Levando essas derivadas na expressão $\sum_{j=m}^{\infty} \frac{\partial f_{k}}{\partial x_{j}}(\bar{x}) v_{j}$, obtemos:

$$
\begin{gathered}
\left|D F_{k}(\bar{x})\left(0, \ldots, 0, v_{m}, v_{m+1}, \ldots\right)\right| \leq_{c . c .} \\
\leq_{c . c .} \underbrace{\underbrace{m+j)^{s}}_{\beta_{k}}, \quad 1 \leq k \leq m-1 .}_{\sum_{j=m-k}^{m-1}\left(\begin{array}{c}
0 \\
0 \\
\left|\bar{a}_{j}^{3}\right|+\left|\bar{b}_{j}^{3}\right|+\left|\bar{a}_{j}^{1}\right|+\left|\bar{b}_{j}^{1}\right| \\
\left|\bar{a}_{j}^{3}\right|+\left|\bar{b}_{j}^{3}\right|+\left|\bar{a}_{j}^{1}\right|+\left|\bar{b}_{j}^{1}\right| \\
\left|\bar{a}_{j}^{2}\right|+\left|\bar{b}_{j}^{2}\right|+\left|\bar{a}_{j}^{1}\right|+\left|\bar{b}_{j}^{1}\right| \\
\left|\bar{a}_{j}^{2}\right|+\left|\bar{b}_{j}^{2}\right|+\left|\bar{a}_{j}^{1}\right|+\left|\bar{b}_{j}^{1}\right|
\end{array}\right)}
\end{gathered}
$$

E ainda:

$$
D f_{0}(\bar{x})\left(0, \ldots, 0, v_{m}, v_{m+1}, \ldots\right)=\left(\begin{array}{l}
0 \\
0 \\
0 \\
0
\end{array}\right)=\beta_{0}
$$

Defina

$$
B=\left(\begin{array}{c}
\beta_{0} \\
\beta_{1} \\
\vdots \\
\beta_{m-1}
\end{array}\right)
$$

Substituindo as estimativas acima na desigualdade (4.17) obtemos

$$
\left|(D T(\bar{x}+r u) \cdot r v)^{m}\right| \leq_{c . c}\left|I d-A^{m} D F^{m}(\bar{c})\right|\left|v^{m}\right| r+\left|A^{m}\right| B r+\left|A^{m}\right| E r^{2} .
$$

Logo, podemos tomar

$$
(Z(r))^{m}=\left[\left|I d-A^{m} D F^{m}(\bar{c})\right| w^{m}+\left|A^{m}\right| \cdot B\right] r+\left|A^{m}\right| \cdot E r^{2},
$$


onde

$$
w=\left\{\omega_{k}^{-s}\right\}_{k \geq 0} .
$$

Resta estimar os termos $(D T(\bar{x}+r u) \cdot r v)_{k}$ para $k \geq m$. Nesse sentido, podemos escrever:

$$
\begin{aligned}
& (D T(\bar{x}+r u) \cdot r v)_{k}=D T_{k}(\bar{x}+r u) \cdot r v=r v_{k}-\left[\frac{\partial f_{k}}{\partial x_{k}}(\bar{x})\right]^{-1} \cdot\left[D f_{k}(\bar{x}+r u) r v\right]= \\
= & r v_{k}-\left[\frac{\partial f_{k}}{\partial x_{k}}(\bar{x})\right]^{-1} \cdot\left[D f_{k}(\bar{x}) r v\right]-\left[\frac{\partial f_{k}}{\partial x_{k}}(\bar{x})\right]^{-1} \cdot\left[D f_{k}(\bar{x}+r u) r v-D f_{k}(\bar{x}) r v\right]= \\
= & r v_{k}-\left[\frac{\partial f_{k}}{\partial x_{k}}(\bar{x})\right]^{-1} \cdot \sum_{j=0}^{\infty} \frac{\partial F_{k}}{\partial x_{j}}(\bar{x}) r v_{j}-\left[\frac{\partial f_{k}}{\partial x_{k}}(\bar{x})\right]^{-1} \cdot\left[D f_{k}(\bar{x}+r u) r v-D f_{k}(\bar{x}) r v\right]= \\
= & -\left[\frac{\partial f_{k}}{\partial x_{k}}(\bar{x})\right]^{-1} \cdot \sum_{j=0, j \neq k}^{\infty} \frac{\partial F_{k}}{\partial x_{j}}(\bar{x}) r v_{j}-\left[\frac{\partial f_{k}}{\partial x_{k}}(\bar{x})\right]^{-1} \cdot\left[D f_{k}(\bar{x}+r u) r v-D f_{k}(\bar{x}) r v\right] \cdot(4.25)
\end{aligned}
$$

Para estimar a primeira parcela da soma do lado direito de (4.25) basta usar o Corolário 4.2.1, e as expressões

$$
\frac{\partial f_{k}}{\partial x_{j}}(\bar{x})=\left(\begin{array}{cccccc}
0 & 0 & 0 & 0 & 0 & 0 \\
0 & 0 & 0 & 0 & 0 & 0 \\
-\bar{a}_{k-j}^{3} & \bar{b}_{k-j}^{3} & 0 & 0 & -\bar{a}_{k-j}^{1} & -\bar{b}_{k-j}^{1} \\
-\bar{b}_{k-j}^{3} & -\bar{a}_{k-j}^{3} & 0 & 0 & -\bar{b}_{k-j}^{1} & -\bar{a}_{k-j}^{1} \\
\bar{a}_{k-j}^{2} & -\bar{b}_{k-j}^{2} & \bar{a}_{k-j}^{1} & -\bar{b}_{k-j}^{1} & 0 & 0 \\
\bar{b}_{k-j}^{2} & \bar{a}_{k-j}^{2} & \bar{b}_{k-j}^{1} & -\bar{a}_{k-j}^{1} & 0 & 0
\end{array}\right), \forall k \geq m, j \geq 1, j \neq k
$$

e

$$
\frac{\partial f_{k}}{\partial x_{0}}(\bar{x})=\left(\begin{array}{cccc}
0 & 0 & 0 & 0 \\
0 & 0 & 0 & 0 \\
0 & 0 & 0 & 0 \\
0 & 0 & 0 & 0 \\
0 & 0 & 0 & 0
\end{array}\right), k \geq m .
$$

Para estimar a segunda parcela na soma do lado direito de (4.25) basta usar o Corolário 4.2.1 e as estimativas dadas por (4.22.)

Aplicando esses resultados em (4.25), obtemos:

$$
\begin{aligned}
& \left|(D T(\bar{x}+r u) \cdot r v)_{k}\right| \leq_{c . c .} \frac{C_{m}}{k}\left[\sum_{j=1}^{m-1}\left(\begin{array}{c}
0 \\
0 \\
\left|\bar{a}_{j}^{1}\right|+\left|\bar{b}_{j}^{1}\right|+\left|\bar{a}_{j}^{3}\right|+\left|\bar{b}_{j}^{3}\right| \\
\left|\bar{a}_{j}^{1}\right|+\left|\bar{b}_{j}^{1}\right|+\left|\bar{a}_{j}^{3}\right|+\left|\bar{b}_{j}^{3}\right| \\
\left|\bar{a}_{j}^{1}\right|+\left|\bar{b}_{j}^{1}\right|+\left|\bar{a}_{j}^{2}\right|+\left|\bar{b}_{j}^{2}\right| \\
\left|\bar{a}_{j}^{1}\right|+\left|\bar{b}_{j}^{1}\right|+\left|\bar{a}_{j}^{2}\right|+\left|\bar{b}_{j}^{2}\right|
\end{array}\right) \frac{1}{(k+j)^{s}}\right] r+ \\
& +\frac{C_{m}}{k}\left(\frac{1}{k^{s-1}}+8 \frac{\sigma_{k}}{k^{s}}\right)\left(\begin{array}{c}
1 \\
1 \\
1 \\
1 \\
1 \\
1
\end{array}\right) r^{2}
\end{aligned}
$$


Desse modo, para $k \geq m$ podemos tomar

$$
\begin{gathered}
Z_{k}(r)=\left|(D T(\bar{x}+r u) \cdot r v)_{k}\right| \leq c . c . \frac{C_{m}}{k}\left[\sum_{j=1}^{m-1}\left(\begin{array}{c}
0 \\
0 \\
\left|\bar{a}_{j}^{1}\right|+\left|\bar{b}_{j}^{1}\right|+\left|\bar{a}_{j}^{3}\right|+\left|\bar{b}_{j}^{3}\right| \\
\left|\bar{a}_{j}^{1}\right|+\left|\bar{b}_{j}^{1}\right|+\left|\bar{a}_{j}^{3}\right|+\left|\bar{b}_{j}^{3}\right| \\
\left|\bar{a}_{j}^{1}\right|+\left|\bar{b}_{j}^{1}\right|+\left|\bar{a}_{j}^{2}\right|+\left|\bar{b}_{j}\right| \\
\left|\bar{a}_{j}^{1}\right|+\left|\bar{b}_{j}^{1}\right|+\left|\bar{a}_{j}^{2}\right|+\left|\bar{b}_{j}\right|
\end{array}\right) \frac{1}{(k+j)^{s}}\right] r+ \\
+\frac{C_{m}}{k}\left(\frac{1}{k^{s-1}}+8 \frac{\sigma_{k}}{k^{s}}\right)\left(\begin{array}{c}
1 \\
1 \\
1 \\
1 \\
1 \\
1
\end{array}\right) r^{2} .
\end{gathered}
$$

Teorema 4.2.4. Dado $M \geq 6$ existe polinômio de segundo grau $Z_{M}(r)$ tal que $Z_{k}(r) \leq$ $\frac{Z_{M}(r)}{k^{s}}$ para todo $k \geq M$.

Demonstração. Para $k \geq M$ temos:

$$
\begin{gathered}
\frac{C_{m}}{k}\left[\sum_{j=1}^{m-1}\left(\begin{array}{c}
0 \\
0 \\
\left|\bar{a}_{j}^{1}\right|+\left|\bar{b}_{j}^{1}\right|+\left|\bar{a}_{j}^{3}\right|+\left|\bar{b}_{j}^{3}\right| \\
\left|\bar{a}_{j}^{1}\right|+\left|\bar{b}_{j}^{1}\right|+\left|\bar{a}_{j}^{3}\right|+\left|\bar{b}_{j}^{3}\right| \\
\left|\bar{a}_{j}^{1}\right|+\left|\bar{b}_{j}^{1}\right|+\left|\bar{a}_{j}^{2}\right|+\left|\bar{b}_{j}^{2}\right| \\
\left|\bar{a}_{j}^{1}\right|+\left|\bar{b}_{j}^{1}\right|+\left|\bar{a}_{j}^{2}\right|+\left|\bar{b}_{j}^{2}\right|
\end{array}\right) \frac{1}{(k+j)^{s}}\right] r \leq \\
\leq \frac{1}{k^{s}}\left[\frac{C_{m}}{M} \sum_{j=1}^{m-1}\left(\begin{array}{c}
0 \\
\left|\bar{a}_{j}^{1}\right|+\left|\bar{b}_{j}^{1}\right|+\left|\bar{a}_{j}^{3}\right|+\left|\bar{b}_{j}^{3}\right| \\
\left|\bar{a}_{j}^{1}\right|+\left|\bar{b}_{j}^{1}\right|+\left|\bar{a}_{j}^{3}\right|+\left|\bar{b}_{j}^{3}\right| \\
\left|\bar{a}_{j}^{1}\right|+\left|\bar{b}_{j}^{1}\right|+\left|\bar{a}_{j}^{2}\right|+\left|\bar{b}_{j}^{2}\right| \\
\left|\bar{a}_{j}^{1}\right|+\left|\bar{b}_{j}^{1}\right|+\left|\bar{a}_{j}^{2}\right|+\left|\bar{b}_{j}^{2}\right|
\end{array}\right)\right] r=: \frac{a_{M}}{k^{s}} r .
\end{gathered}
$$

Por outro lado, como $\sigma_{k}(s) \leq \sigma_{M}(s)$ para todo $k \geq M$, temos que

$$
\frac{C_{m}}{k}\left[\frac{1}{k^{s-1}}+8 \frac{\sigma_{k}(s)}{k^{s}}\right] r^{2} \leq \frac{1}{k^{s}}\left[C_{m}+8 \frac{\sigma_{M}}{M}\right] r^{2}=: \frac{b_{M}}{k^{s}} r^{2} .
$$

Logo,

$$
\left\|Z_{k}(r)\right\|_{\infty} \leq \frac{a_{M} r+b_{M} r^{2}}{k^{s}}=: \frac{Z_{M}(r)}{k^{s}}
$$

\subsubsection{Cálculo de Y}

Teorema 4.2.5. $(T(\bar{x})-\bar{x})_{k}=0$ para todo $k \geq M$.

Demonstração. Temos

$$
|T(\bar{x})-\bar{x}|=\mid \bar{x}-A F(\bar{x}-\bar{x}|=| A F(\bar{x}) \mid .
$$


Logo,

$$
\begin{gathered}
(T(\bar{x})-\bar{x})^{m}=A^{m} F^{m}(\bar{x}) ; \\
(T(\bar{x})-\bar{x})_{k}=\Lambda_{k}^{-1}(x) f_{k}(\bar{x}), k \geq m .
\end{gathered}
$$

Por outro lado,

$$
f_{k}(\bar{x})=\left(\begin{array}{cccccc}
-\sigma & -k \bar{l} & \sigma & 0 & 0 & 0 \\
k \bar{l} & -\sigma & 0 & \sigma & 0 & 0 \\
\rho & 0 & -1 & -k \bar{l} & 0 & 0 \\
0 & \rho & k \bar{l} & -1 & 0 & 0 \\
0 & 0 & 0 & -\beta & -k \bar{l} & 0 \\
0 & 0 & 0 & 0 & k \bar{l} & -\beta
\end{array}\right) \bar{c}_{k}+\left\{\begin{array}{c}
0 \\
0 \\
-\sum_{k_{1}+k_{2}=k} \bar{a}_{k_{1}}^{1} \bar{a}_{k_{2}}^{3}-\bar{b}_{k_{1}}^{1} \bar{b}_{k_{2}}^{3} \\
-\sum_{k_{1}+k_{2}=k} \bar{a}_{k_{1}}^{1} \bar{b}_{k_{2}}^{3}+\bar{b}_{k_{1}}^{1} \bar{a}_{k_{2}}^{3} \\
\sum_{k_{1}+k_{2}=k} \bar{a}_{k_{1}}^{1} \bar{a}_{k_{2}}^{2}-\bar{b}_{k_{1}}^{1} \bar{b}_{k_{2}}^{2} \\
\sum_{k_{1}+k_{2}=k} \bar{a}_{k_{1}}^{1} \bar{b}_{k_{2}}^{2}+\bar{b}_{k_{1}}^{1} \bar{a}_{k_{2}}^{2}
\end{array}\right\}, k \geq 1
$$

Porém, se $k_{1}+k_{2}=k$, com $k \geq 2 m$, então $k_{1} \geq m$ ou $k_{2} \geq m$. Como $\bar{a}_{k}=\bar{b}_{k}=0$ para todo $k \geq m$, segue que $f_{k}(\bar{x})=0$ para todo $k \geq 2 m$. Como $(T(\bar{x})-\bar{x})_{k}=\Lambda_{k}^{-1}(x) f_{k}(\bar{x}), k \geq$ $m$, segue que

$$
(T(\bar{x})-\bar{x})_{k}=0, \forall k \geq 2 m
$$

Segue do teorema acima que podemos tomar

$$
\begin{gathered}
Y^{m}=A^{m} F^{m}(\bar{x}) . \\
Y_{k}=\Lambda_{k}^{-1}(\bar{x}) F_{k}(\bar{x}), m \leq k \leq 2 m-1 . \\
Y_{k}=0, \quad k \geq 2 m .
\end{gathered}
$$

\subsection{Resultados}

Fixamos os parâmetros $\sigma=10, \beta=8 / 3$ e estudamos o surgimento de órbitas periódicas variando o parâmetro $\rho$, atentando para o surgimento de pontos de bifurcação de Hopf, pois podemos usar o pacote MatCont [3], para o MATLAB, a fim de obtermos órbitas periódicas que emanam destes pontos.

Utilizando o pacote MatCont, para o MATLAB, traçamos o diagrama de bifurcação do sistema de Lorenz mostrado na Figura 4.2.

Para $\rho=24.736842$, detectamos um ponto de bifurcação de Hopf. A partir daí o MatCont calcula numericamente as curvas periódicas em torno deste ponto.

Com auxílio da Função fft do MATLAB, obtemos aproximações para os primeiros coeficientes de fourier de uma dessas curvas periódicas, que denotamos por $\gamma_{0}(t)$. Podemos tomar esses coeficientes como chutes iniciais para executarmos o Método de Newton para a função

$$
\begin{gathered}
F_{m}: \mathbb{R}^{6 m-2} \rightarrow \mathbb{R}^{6 m-2} \\
F^{m}(c)=\left(\eta\left(c, 0_{\infty}\right), f_{0}\left(c, 0_{\infty}\right), f_{1}\left(c, 0_{\infty}\right), f_{2}\left(c, 0_{\infty}\right), f_{3}\left(c, 0_{\infty}\right), \ldots, f_{m-1}\left(c, 0_{\infty}\right)\right),
\end{gathered}
$$

visando-se obter algum $\bar{c} \in \mathbb{R}^{6 m-2)}$ tal que $F^{m}(\bar{c}) \approx 0$. Em seguida, implemtentamos os polinômios $p_{k}(r)=Z_{k}(r)+Y_{k}-\frac{r}{\omega_{k}^{s}}$, para $0 \leq k \leq M$ para $M \geq 2 m$, onde as cotas $Z(r)$ e $Y$ são obtidas como mostrado nas duas seções anteriores. Verificamos a existência de 


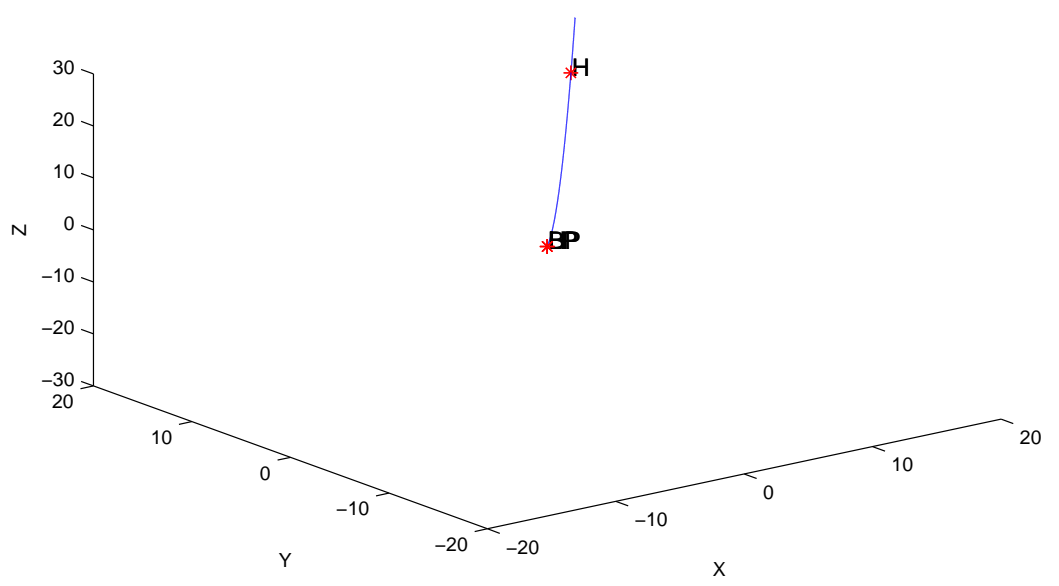

Figura 4.2: Curva de equilíbrios obtida ao variar o parâmetro $\rho$. Uma bifurcação de Hopf (ponto H no gráfico) é encontrada em $\rho=24.736842$.

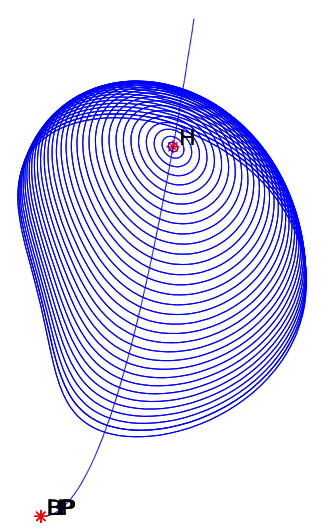

Figura 4.3: Órbitas periódicas emanando da bifurcação de Hopf $(\mathrm{H})$ calculadas numericamente pelo pacote MatCont.

$r>0$ tal que $p_{k}(r)<0$ para todo $0 \leq k \leq M-1$ e $Z_{M}(r)<1$. Refazemos os cálculos com aritmética de intervalo para garantir a validade das operações numéricas.

Como visto ao longo deste trabalho, a existência de $r>0$ satisfazendo essas desigualdades garante a existência de órbitas periódicas próximas às órbitas numéricas.

Tomamos a curva periódica numérica obtida pelo MatCont para o valor de parâmetro $\rho=18$. Aplicando a Função fft a essa curva, obtemos alguns de seus coeficientes de Fourier, que estão apresentados na Tabela 4.1, onde $c_{k}=a_{k}+i b_{k}$ é o k-ésimo coeficiente retornado pela Função fft e $l$ é a frequência da solução que estes coeficientes representam (se a frequência é $l$ o período da solução é $2 \pi / l$ ).

A Função fft retorna tantos coeficientes de Fourier quantos forem os pontos da curva calculados pelo MatCont. Na Tabela 4.1 selecionamos apenas os $m=20$ primeiros coeficientes. Com este valor de $m$ aplicamos o Método de Newton à função $F^{m}$, com chute inicial dado por esses $m$ coeficientes. Com isso, esperamos obter, via Método de Newton, uma nova solução periódica aproximada, para a qual sabemos provar, com o método dos polinômios radiais, a existência de uma órbita periódica nas suas proximidades. A solução $\sum_{-m<k<m} \bar{c}_{k} e^{-i k \bar{l} t}$ obtida desta maneira tem os coeficientes de Fourier apresentados na 
Tabela 4.2 .

Na Figura 4.4 apresentamos um esboço da órbita associada a estes coeficientes.

Para a solução numérica assim obtida, o método dos polinômios radiais prova o seguinte teorema.

Teorema 4.3.1. Para $\rho=18$, sejam $s=2.1$ e $r=2.2671 \times 10^{-4}$. Existe um único ponto $\hat{c} \in B_{\Omega^{s}}[\bar{c}, r]$ tal que $F(\hat{c})=0$.

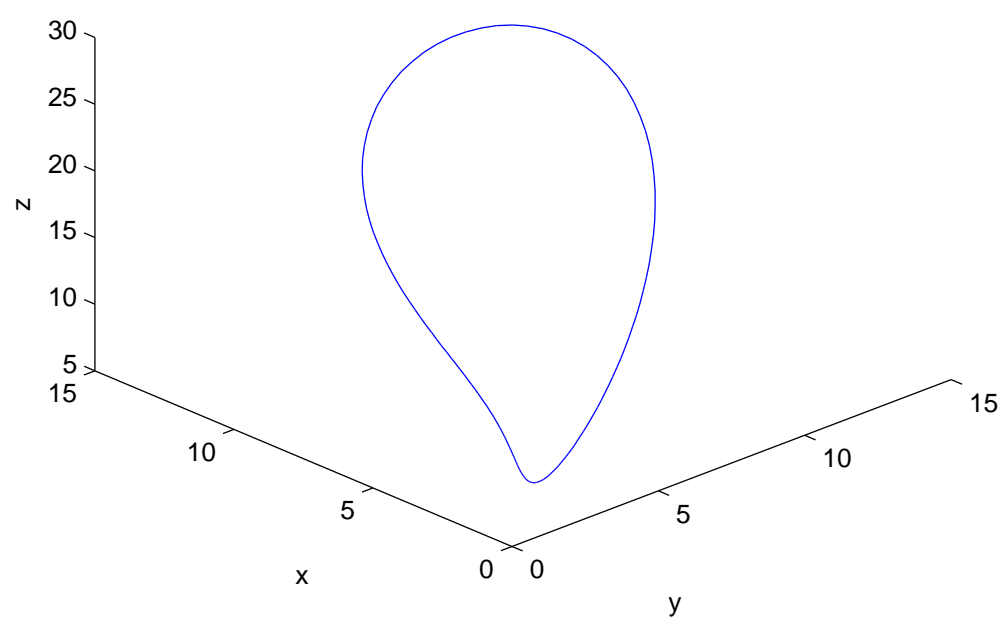

Figura 4.4: Órbita associada à solução numérica $\bar{c}$, obtida pelo Método de Newton para o parâmetro $\rho=18$.

O teorema acima pode ser obtido para os valores do parâmetro $\rho$, variando entre $\rho=15$ $\rho=24$. Na Figura 4.5, apresentamos as órbitas obtidas pelo método aqui apresentado ao variar o parâmetro $\rho$ neste intervalo.

Pode-se ainda aplicar o método descrito neste capítulo com a condição de fase dada no capítulo anterior, na qual impomos que algum dos coeficientes tenha parte real nula. Para $\rho=23.7715$, tomando-se $m=41$ no Método de Newton com a condição de fase $\operatorname{Re}\left\{c_{1}^{1}\right\}=0$ obtemos, a partir de um chute inicial aleatória, convergência para uma raiz aproximada de $F^{m}$ cujos coeficientes não nulos estão dados na Tabela 4.3.

A Figura 4.6 mostra um esboço da órbita construída a partir destes coeficientes.

Teorema 4.3.2. Seja $\bar{c}$ como dado pela Tabela 4.3, s $=2.1$ e $r=6.0750 \times 10^{-4}$. Existe único ponto $\hat{c} \in B_{\Omega^{s}}[\bar{c}, r]$ tal que $\gamma(t)=\sum_{k \in \mathbb{Z}} \hat{c}_{k} e^{-i k \hat{l} t}$ é solução periódica do sistema de Lorenz para o parâmetro $\rho=23.7715$.

Para cada $\rho$ no intervalo $[15,24]$ obtemos, pelo método dos polinômios radiais, um teorema análogo ao enunciado acima. Na Figura 4.7 traçamos a curva de soluções obtida ao variar $\rho$ neste intervalo. 


\begin{tabular}{|c|c|c|c|}
\hline$l=-6.4023$ & $a_{5}^{2}=5.9663 \times 10^{-3}$ & $a_{10}^{2}=-5.8828 \times 10^{-5}$ & $a_{15}^{2}=1.3850 \times 10^{-7}$ \\
\hline$a_{0}^{1}=4.8649$ & $b_{5}^{2}=-4.7491 \times 10^{-2}$ & $b_{10}^{2}=7.8028 \times 10^{-5}$ & $b_{15}^{2}=-7.2174 \times 10^{-8}$ \\
\hline$a_{0}^{2}=4.8649$ & $a_{5}^{3}=-4.6882 \times 10^{-2}$ & $a_{10}^{3}=7.7473 \times 10^{-5}$ & $a_{15}^{3}=-7.1358 \times 10^{-8}$ \\
\hline$a_{0}^{3}=14.322$ & $b_{5}^{3}=-6.8238 \times 10^{-3}$ & $b_{10}^{3}=5.9440 \times 10^{-5}$ & $b_{15}^{3}=-1.3884 \times 10^{-7}$ \\
\hline$a_{1}^{1}=-1.4779$ & $a_{6}^{1}=2.6185 \times 10^{-3}$ & $a_{11}^{1}=-3.5692 \times 10^{-6}$ & $a_{16}^{1}=4.1219 \times 10^{-9}$ \\
\hline$b_{1}^{1}=2.0858$ & $b_{6}^{1}=-2.4880 \times 10^{-3}$ & $b_{11}^{1}=1.4156 \times 10^{-6}$ & $b_{16}^{1}=6.8619 \times 10^{-11}$ \\
\hline$a_{1}^{2}=-2.8133$ & $a_{6}^{2}=1.2176 \times 10^{-2}$ & $a_{11}^{2}=-1.3538 \times 10^{-5}$ & $a_{16}^{2}=3.4190 \times 10^{-9}$ \\
\hline$b_{1}^{\frac{1}{2}}=1.1397$ & $b_{6}^{2}=7.5707 \times 10^{-3}$ & $b_{11}^{2}=-2.3720 \times 10^{-5}$ & $b_{16}^{2}=4.2292 \times 10^{-8}$ \\
\hline$a_{1}^{3}=1.2773$ & $a_{6}^{3}=7.7461 \times 10^{-3}$ & $a_{11}^{3}=-2.3807 \times 10^{-5}$ & $a_{16}^{3}=4.2292 \times 10^{-8}$ \\
\hline$b_{1}^{3}=4.2514$ & $b_{6}^{3}=-1.1997 \times 10^{-2}$ & $b_{11}^{3}=1.3348 \times 10^{-5}$ & $b_{16}^{3}=-3.1932 \times 10^{-9}$ \\
\hline$a_{2}^{1}=-4.6848 \times 10^{-1}$ & $a_{7}^{1}=3.3304 \times 10^{-4}$ & $a_{12}^{1}=1.4710 \times 10^{-8}$ & $a_{17}^{1}=-4.1883 \times 10^{-10}$ \\
\hline$b_{2}^{1}=-6.7494 \times 10^{-1}$ & $b_{7}^{1}=8.5387 \times 10^{-4}$ & $b_{12}^{1}=-9.7780 \times 10^{-7}$ & $b_{17}^{1}=9.6363 \times 10^{-10}$ \\
\hline$a_{2}^{2}=3.9574 \times 10^{-1}$ & $a_{7}^{2}=-3.4936 \times 10^{-3}$ & $a_{12}^{2}=7.5268 \times 10^{-6}$ & $a_{17}^{\frac{1}{2}}=-1.0907 \times 10^{-8}$ \\
\hline$b_{2}^{2}=-1.2748$ & $b_{7}^{2}=2.3464 \times 10^{-3}$ & $b_{12}^{2}=-8.6478 \times 10^{-7}$ & $b_{17}^{2}=-3.5948 \times 10^{-9}$ \\
\hline$a_{2}^{3}=-1.3639$ & $a_{7}^{3}=2.2951 \times 10^{-3}$ & $a_{12}^{3}=-8.1149 \times 10^{-7}$ & $a_{17}^{3}=-3.6481 \times 10^{-9}$ \\
\hline$b_{2}^{3}=-3.5767 \times 10^{-1}$ & $b_{7}^{3}=3.5198 \times 10^{-3}$ & $b_{12}^{3}=-7.5280 \times 10^{-6}$ & $b_{17}^{3}=1.0885 \times 10^{-8}$ \\
\hline$a_{3}^{1}=2.1213 \times 10^{-1}$ & $a_{8}^{1}=-2.3289 \times 10^{-4}$ & $a_{13}^{1}=2.2868 \times 10^{-7}$ & $a_{18}^{1}=-1.8582 \times 10^{-10}$ \\
\hline$b_{3}^{1}=-6.3447 \times 10^{-2}$ & $b_{8}^{1}=-4.2179 \times 10^{-6}$ & $b_{13}^{1}=9.8824 \times 10^{-8}$ & $b_{18}^{1}=-1.9266 \times 10^{-10}$ \\
\hline$a_{3}^{2}=3.3400 \times 10^{-1}$ & $a_{8}^{2}=-2.1129 \times 10^{-4}$ & $a_{13}^{2}=-5.9383 \times 10^{-7}$ & $a_{18}^{2}=2.0344 \times 10^{-9}$ \\
\hline$b_{3}^{2}=3.4400 \times 10^{-1}$ & $b_{8}^{2}=-1.1970 \times 10^{-3}$ & $b_{13}^{2}=2.0021 \times 10^{-6}$ & $b_{18}^{2}=-2.3341 \times 10^{-9}$ \\
\hline$a_{3}^{3}=3.3379 \times 10^{-1}$ & $a_{8}^{3}=-1.1983 \times 10^{-3}$ & $a_{13}^{3}=1.9971 \times 10^{-6}$ & $a_{18}^{3}=-2.3238 \times 10^{-9}$ \\
\hline$b_{3}^{3}=-3.3082 \times 10^{-1}$ & $b_{8}^{3}=1.9779 \times 10^{-4}$ & $b_{13}^{3}=6.0646 \times 10^{-7}$ & $b_{18}^{3}=-2.0452 \times 10^{-9}$ \\
\hline$a_{4}^{1}=-3.1781 \times 10^{-3}$ & $a_{9}^{1}=2.3574 \times 10^{-5}$ & $a_{14}^{1}=-4.5576 \times 10^{-8}$ & $a_{19}^{1}=6.3144 \times 10^{-11}$ \\
\hline$b_{4}^{1}=5.6401 \times 10^{-2}$ & $b_{9}^{1}=-5.4360 \times 10^{-5}$ & $b_{14}^{1}=4.4178 \times 10^{-8}$ & $b_{19}^{1}=-2.4723 \times 10^{-11}$ \\
\hline$a_{4}^{2}=-1.4761 \times 10^{-1}$ & $a_{9}^{2}=3.3680 \times 10^{-4}$ & $a_{14}^{2}=-4.4155 \times 10^{-7}$ & $a_{19}^{2}=3.6389 \times 10^{-10}$ \\
\hline$b_{4}^{2}=4.8262 \times 10^{-2}$ & $b_{9}^{2}=8.1473 \times 10^{-5}$ & $b_{14}^{2}=-3.6433 \times 10^{-7}$ & $b_{19}^{2}=7.4337 \times 10^{-10}$ \\
\hline$a_{4}^{3}=4.5182 \times 10^{-2}$ & $a_{9}^{3}=8.4606 \times 10^{-5}$ & $a_{14}^{3}=-3.6680 \times 10^{-7}$ & $a_{19}^{3}=7.4580 \times 10^{-10}$ \\
\hline$b_{4}^{3}=1.4529 \times 10^{-1}$ & $b_{9}^{3}=-3.3576 \times 10^{-4}$ & $b_{14}^{3}=4.3912 \times 10^{-7}$ & $b_{19}^{3}=-3.6006 \times 10^{-10}$ \\
\hline$a_{5}^{1}=-1.2986 \times 10^{-2}$ & $a_{10}^{1}=1.0496 \times 10^{-5}$ & $a_{15}^{1}=-5.9492 \times 10^{-9}$ & \\
\hline$b_{5}^{1}=-5.9206 \times 10^{-3}$ & $b_{10}^{1}=1.0828 \times 10^{-5}$ & $b_{15}^{1}=-1.5041 \times 10^{-8}$ & \\
\hline
\end{tabular}

Tabela 4.1: Chute inicial para o Método de Newton, obtido via pacote MatCont e Função fft.

\begin{tabular}{|c|c|c|c|}
\hline $\bar{l}=-6.0663$ & $\bar{a}_{5}^{2}=-3.9221 \times 10^{-2}$ & $\bar{a}_{10}^{2}=1.5000 \times 10^{-4}$ & $\bar{a}_{15}^{2}-4.1666 \times 10^{-7}$ \\
\hline $\bar{a}_{0}^{1}=4.5651$ & $\bar{b}_{5}^{2}=-4.9572 \times 10^{-2}$ & $\bar{b}_{10}^{2}=1.1254 \times 10^{-4}$ & $\bar{b}_{15}^{2}=-1.3227 \times 10^{-7}$ \\
\hline $\bar{a}_{0}^{2}=4.5651$ & $\bar{a}_{5}^{3}=-4.9715 \times 10^{-2}$ & $\bar{a}_{10}^{3}=1.1379 \times 10^{-4}$ & $\bar{a}_{15}^{3}=-1.3468 \times 10^{-7}$ \\
\hline $\bar{a}_{0}^{3}=13.407$ & $\bar{b}_{5}^{3}=3.7775 \times 10^{-2}$ & $\bar{b}_{10}^{3}=-1.4888 \times 10^{-4}$ & $\bar{b}_{15}^{3}=4.1566 \times 10^{-7}$ \\
\hline $\bar{a}_{1}^{1}=-1.1336$ & $\bar{a}_{6}^{1}=-6.7789 \times 10^{-4}$ & $\bar{a}_{11}^{1}=4.2216 \times 10^{-6}$ & $\bar{a}_{16}^{1}=-1.0422 \times 10^{-8}$ \\
\hline $\bar{b}_{1}^{1}=2.3007$ & $\bar{b}_{6}^{1}=-5.3590 \times 10^{-3}$ & $\bar{b}_{11}^{1}=7.2343 \times 10^{-6}$ & $\bar{b}_{16}^{1}=-7.9810 \times 10^{-9}$ \\
\hline $\bar{a}_{1}^{2}=-2.5293$ & $\bar{a}_{6}^{2}=1.8828 \times 10^{-2}$ & $\bar{a}_{11}^{2}=-4.4053 \times 10^{-5}$ & $\bar{a}_{16}^{2}=6.7042 \times 10^{-8}$ \\
\hline $\bar{b}_{1}^{2}=1.6130$ & $\bar{b}_{6}^{2}=-7.8264 \times 10^{-3}$ & $\bar{b}_{11}^{2}=3.5405 \times 10^{-5}$ & $\bar{b}_{16}^{2}=-1.0914 \times 10^{-7}$ \\
\hline $\bar{a}_{1}^{3}=1.9497$ & $\bar{a}_{6}^{3}=-7.4467 \times 10^{-3}$ & $\bar{a}_{11}^{3}=3.5023 \times 10^{-5}$ & $\bar{a}_{16}^{3}=-1.0872 \times 10^{-7}$ \\
\hline $\bar{b}_{1}^{3}=4.0291$ & $\bar{b}_{6}^{3}=-1.8867 \times 10^{-2}$ & $\bar{b}_{11}^{3}=4.4304 \times 10^{-5}$ & $\bar{b}_{16}^{3}=-6.7621 \times 10^{-8}$ \\
\hline $\bar{a}_{2}^{1}=-7.0839 \times 10^{-1}$ & $\bar{a}_{7}^{1}=1.4697 \times 10^{-3}$ & $\bar{a}_{12}^{1}=-2.2032 \times 10^{-6}$ & $\bar{a}_{17}^{1}=2.8048 \times 10^{-9}$ \\
\hline $\bar{b}_{2}^{1}=-5.4865 \times 10^{-1}$ & $\bar{b}_{7}^{1}=1.5457 \times 10^{-4}$ & $\bar{b}_{12}^{1}=6.6305 \times 10^{-7}$ & $\bar{b}_{17}^{1}=-2.2706 \times 10^{-9}$ \\
\hline $\bar{a}_{2}^{2}=-4.2730 \times 10^{-2}$ & $\bar{a}_{7}^{2}=8.1338 \times 10^{-4}$ & $\bar{a}_{12}^{2}=-7.0300 \times 10^{-6}$ & $\bar{a}_{17}^{2}=2.6221 \times 10^{-8}$ \\
\hline $\bar{b}_{2}^{2}=-1.4081$ & $\bar{b}_{7}^{2}=6.3957 \times 10^{-3}$ & $\bar{b}_{12}^{2}=-1.5376 \times 10^{-5}$ & $\bar{b}_{17}^{2}=2.6655 \times 10^{-8}$ \\
\hline $\bar{a}_{2}^{3}=-1.5038$ & $\bar{a}_{7}^{3}=6.3938 \times 10^{-3}$ & $\bar{a}_{12}^{3}=-1.5417 \times 10^{-5}$ & $\bar{a}_{17}^{3}=2.6783 \times 10^{-8}$ \\
\hline $\bar{b}_{2}^{3}=1.1008 \times 10^{-1}$ & $\bar{b}_{7}^{3}=-7.1786 \times 10^{-4}$ & $\bar{b}_{12}^{3}=6.9116 \times 10^{-6}$ & $\bar{b}_{17}^{3}=-2.6070 \times 10^{-8}$ \\
\hline $\bar{a}_{3}^{1}=1.9204 \times 10^{-1}$ & $\bar{a}_{8}^{1}=-1.3423 \times 10^{-4}$ & $\bar{a}_{13}^{1}=-3.5460 \times 10^{-8}$ & $\bar{a}_{18}^{1}=4.2555 \times 10^{-10}$ \\
\hline $\bar{b}_{3}^{1}=-1.7914 \times 10^{-1}$ & $\bar{b}_{8}^{1}=3.8214 \times 10^{-4}$ & $\bar{b}_{13}^{1}=-6.3120 \times 10^{-7}$ & $\bar{b}_{18}^{1}=8.9543 \times 10^{-10}$ \\
\hline $\bar{a}_{3}^{2}=5.1805 \times 10^{-1}$ & $\bar{a}_{8}^{2}=-1.9888 \times 10^{-3}$ & $\bar{a}_{13}^{2}=4.9423 \times 10^{-6}$ & $\bar{a}_{18}^{2}=-9.3519 \times 10^{-9}$ \\
\hline $\bar{b}_{3}^{2}=1.7036 \times 10^{-1}$ & $\bar{b}_{8}^{2}=-2.6928 \times 10^{-4}$ & $\bar{b}_{13}^{2}=-9.1084 \times 10^{-7}$ & $\bar{b}_{18}^{2}=5.5421 \times 10^{-9}$ \\
\hline $\bar{a}_{3}^{3}=1.6010 \times 10^{-1}$ & $\bar{a}_{8}^{3}=-2.9277 \times 10^{-4}$ & $\bar{a}_{13}^{3}=-8.7651 \times 10^{-7}$ & $\bar{a}_{18}^{3}=5.4932 \times 10^{-9}$ \\
\hline $\bar{b}_{3}^{3}=-5.1174 \times 10^{-1}$ & $\bar{b}_{8}^{3}=1.9833 \times 10^{-3}$ & $\bar{b}_{13}^{3}=-4.9454 \times 10^{-6}$ & $\bar{b}_{18}^{3}=9.3781 \times 10^{-9}$ \\
\hline $\bar{a}_{4}^{1}=3.8733 \times 10^{-2}$ & $\bar{a}_{9}^{1}=-9.3425 \times 10^{-5}$ & $\bar{a}_{14}^{1}=1.7092 \times 10^{-7}$ & $\bar{a}_{19}^{1}=-2.6493 \times 10^{-10}$ \\
\hline $\bar{b}_{4}^{1}=6.1360 \times 10^{-2}$ & $\bar{b}_{9}^{1}=-6.0151 \times 10^{-5}$ & $\bar{b}_{14}^{1}=3.1153 \times 10^{-8}$ & $\bar{b}_{19}^{1}=5.5382 \times 10^{-11}$ \\
\hline $\bar{a}_{4}^{2}=-1.1016 \times 10^{-1}$ & $\bar{a}_{9}^{2}=2.3498 \times 10^{-4}$ & $\bar{a}_{14}^{2}=-9.3655 \times 10^{-8}$ & $\bar{a}_{19}^{2}=-9.0326 \times 10^{-10}$ \\
\hline $\bar{b}_{4}^{2}=1.5535 \times 10^{-1}$ & $\bar{b}_{9}^{2}=-5.7022 \times 10^{-4}$ & $\bar{b}_{14}^{2}=1.4828 \times 10^{-6}$ & $\bar{b}_{19}^{2}=-2.9982 \times 10^{-9}$ \\
\hline $\bar{a}_{4}^{3}=1.5064 \times 10^{-1}$ & $\bar{a}_{9}^{3}=-5.6736 \times 10^{-4}$ & $\bar{a}_{14}^{3}=1.4813 \times 10^{-6}$ & $\bar{a}_{19}^{3}=-3.0058 \times 10^{-9}$ \\
\hline $\bar{b}_{4}^{3}=1.0960 \times 10^{-1}$ & $\bar{b}_{9}^{3}=-2.4056 \times 10^{-4}$ & $\bar{b}_{14}^{3}=1.0303 \times 10^{-7}$ & $\bar{b}_{19}^{3}=8.8620 \times 10^{-10}$ \\
\hline $\bar{a}_{5}^{1}=-1.8586 \times 10^{-2}$ & $\bar{a}_{10}^{1}=2.2029 \times 10^{-5}$ & $\bar{a}_{15}^{1}=-1.9334 \times 10^{-8}$ & \\
\hline $\bar{b}_{5}^{1}=6.8031 \times 10^{-3}$ & $\bar{b}_{10}^{\mathrm{I}}=-2.1096 \times 10^{-5}$ & $\bar{b}_{15}^{1}=4.3665 \times 10^{-8}$ & \\
\hline
\end{tabular}

Tabela 4.2: Solução obtida via Método de Newton com chute inicial expresso na tabela acima. 


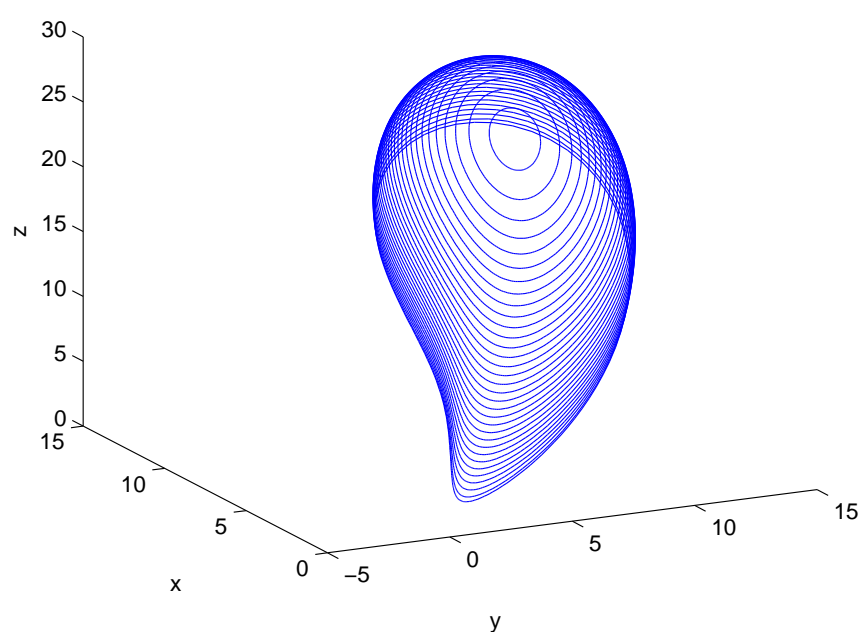

Figura 4.5: Curva de soluções periódicas do sistema de Lorenz obtida ao vairar o parâmetro $\rho$ no interalo $[15,24]$ com passo 0.1 .

\begin{tabular}{|c|c|c|c|}
\hline \multicolumn{4}{|c|}{$\bar{l}=3.5677$} \\
\hline $\bar{a}_{0}^{3}=18.688$ & $\bar{a}_{11}^{1}=5.3617 \times 10^{-3}$ & $\bar{a}_{21}^{1}=-1.6485 \times 10^{-5}$ & $\bar{a}_{31}^{2}=-1.9867 \times 10^{-7}$ \\
\hline $\bar{a}_{1}^{1}=1.5407$ & $\bar{b}_{11}^{1}=9.9694 \times 10^{-3}$ & $\bar{b}_{21}^{1}=-1.4786 \times 10^{-6}$ & $\bar{b}_{31}^{2}=-1.8527 \times 10^{-7}$ \\
\hline $\bar{b}_{1}^{1}=-4.3186$ & $\bar{a}_{11}^{2}=4.4486 \times 10^{-2}$ & $\bar{a}_{21}^{2}=-2.7564 \times 10^{-5}$ & $\bar{a}_{31}^{3}=-4.3128 \times 10^{-8}$ \\
\hline $\bar{b}_{1}^{2}=-4.8683$ & $\bar{b}_{11}^{2}=-1.1072 \times 10^{-2}$ & $\bar{b}_{21}^{2}=1.2203 \times 10^{-4}$ & $\bar{b}_{32}^{3}=-1.3957 \times 10^{-7}$ \\
\hline $\bar{a}_{2}^{3}=0.89939$ & $\bar{a}_{12}^{3}=2.4991 \times 10^{-2}$ & $\bar{a}_{22}^{3}=-4.5874 \times 10^{-5}$ & $\bar{a}_{33}^{1}=-6.5761 \times 10^{-9}$ \\
\hline $\bar{b}_{2}^{3}=-5.4915$ & $\bar{b}_{12}^{3}=6.4471 \times 10^{-3}$ & $\bar{b}_{22}^{3}=5.0459 \times 10^{-5}$ & $\bar{b}_{33}^{\perp}=-9.6614 \times 10^{-10}$ \\
\hline $\bar{a}_{3}^{1}=-1.8496$ & $\bar{a}_{13}^{1}=1.6475 \times 10^{-3}$ & $\bar{a}_{23}^{1}=1.8501 \times 10^{-6}$ & $\bar{a}_{33}^{2}=-1.7951 \times 10^{-8}$ \\
\hline $\bar{b}_{3}^{1}=0.34898$ & $\bar{b}_{13}^{1}=-2.5792 \times 10^{-3}$ & $\bar{b}_{23}^{1}=4.0951 \times 10^{-6}$ & $\bar{b}_{33}^{2}=7.6456 \times 10^{-8}$ \\
\hline $\bar{a}_{3}^{2}=-1.4761$ & $\bar{a}_{13}^{2}=-1.0315 \times 10^{-2}$ & $\bar{a}_{23}^{2}=3.5453 \times 10^{-5}$ & $\bar{a}_{34}^{3}=-2.8790 \times 10^{-8}$ \\
\hline $\bar{b}_{3}^{2}=2.3286$ & $\bar{b}_{13}^{2}=-1.0220 \times 10^{-2}$ & $\bar{b}_{23}^{2}=-1.1086 \times 10^{-5}$ & $\bar{b}_{34}^{3}=3.0795 \times 10^{-8}$ \\
\hline $\bar{a}_{4}^{3}=-1.6510$ & $\bar{a}_{14}^{3}=-2.2903 \times 10^{-3}$ & $\bar{a}_{24}^{3}=1.9715 \times 10^{-5}$ & $\bar{a}_{35}^{1}=6.5079 \times 10^{-10}$ \\
\hline $\bar{b}_{4}^{3}=0.97303$ & $\bar{b}_{14}^{3}=-7.7742 \times 10^{-3}$ & $\bar{b}_{24}^{3}=4.2852 \times 10^{-6}$ & $\bar{b}_{35}^{1}=1.6847 \times 10^{-9}$ \\
\hline $\bar{a}_{5}^{1}=0.32851$ & $\bar{a}_{15}^{1}=-8.2765 \times 10^{-4}$ & $\bar{a}_{25}^{1}=7.2134 \times 10^{-7}$ & $\bar{a}_{35}^{2}=2.1688 \times 10^{-8}$ \\
\hline $\bar{b}_{5}^{1}=0.46407$ & $\bar{b}_{15}^{1}=-4.7485 \times 10^{-5}$ & $\bar{b}_{25}^{1}=-9.8428 \times 10^{-7}$ & $\bar{b}_{35}^{2}=-6.4416 \times 10^{-9}$ \\
\hline $\bar{a}_{5}^{2}=1.1563$ & $\bar{a}_{15}^{2}=-1.0818 \times 10^{-3}$ & $\bar{a}_{26}^{3}=-1.8007 \times 10^{-6}$ & $\bar{a}_{36}^{3}=1.1796 \times 10^{-8}$ \\
\hline $\bar{b}_{5}^{2}=-0.12193$ & $\bar{b}_{15}^{2}=4.3817 \times 10^{-3}$ & $\bar{b}_{26}^{3}=-5.6506 \times 10^{-6}$ & $\bar{b}_{36}^{3}=2.7982 \times 10^{-9}$ \\
\hline $\bar{a}_{6}^{3}=0.64174$ & $\bar{a}_{16}^{3}=-1.7046 \times 10^{-3}$ & $\bar{a}_{27}^{1}=-3.2914 \times 10^{-7}$ & $\bar{a}_{37}^{1}=3.1140 \times 10^{-10}$ \\
\hline $\bar{b}_{6}^{3}=0.26041$ & $\bar{b}_{16}^{3}=1.8283 \times 10^{-3}$ & $\bar{a}_{21}^{1}=-1.6485 \times 10^{-5}$ & $\bar{b}_{37}^{\mathrm{I}}=-3.7913 \times 10^{-10}$ \\
\hline $\bar{a}_{7}^{1}=0.074508$ & $\bar{a}_{17}^{1}=9.8770 \times 10^{-5}$ & $\bar{b}_{21}^{1}=-1.4786 \times 10^{-6}$ & $\bar{a}_{37}^{2}=-4.6933 \times 10^{-9}$ \\
\hline $\bar{b}_{7}^{1}=-0.13705$ & $\bar{b}_{17}^{1}=2.0192 \times 10^{-4}$ & $\bar{a}_{21}^{2}=-2.7564 \times 10^{-5}$ & $\bar{b}_{37}^{2}=-4.4898 \times 10^{-9}$ \\
\hline $\bar{a}_{7}^{2}=-0.26775$ & $\bar{a}_{17}^{2}=1.3234 \times 10^{-3}$ & $\bar{b}_{21}^{2}=1.2203 \times 10^{-4}$ & $\bar{a}_{38}^{3}=-9.7891 \times 10^{-10}$ \\
\hline $\bar{b}_{7}^{2}=-0.32312$ & $\bar{b}_{17}^{2}=-3.9713 \times 10^{-4}$ & $\bar{a}_{21}^{1}=-1.6485 \times 10^{-5}$ & $\bar{b}_{38}^{3}=-3.3288 \times 10^{-9}$ \\
\hline $\bar{a}_{8}^{3}=-0.049932$ & $\bar{a}_{18}^{3}=7.4203 \times 10^{-4}$ & $\bar{b}_{21}^{1}=-1.4786 \times 10^{-6}$ & $\bar{a}_{39}^{1}=-1.3095 \times 10^{-10}$ \\
\hline $\bar{b}_{8}^{3}=-0.23567$ & $\bar{b}_{18}^{3}=1.6600 \times 10^{-4}$ & $\bar{b}_{29}^{1}=8.3069 \times 10^{-8}$ & $\bar{b}_{39}^{1}=-2.2896 \times 10^{-11}$ \\
\hline $\bar{a}_{9}^{\perp}=-0.041994$ & $\bar{a}_{19}^{\perp}=3.4580 \times 10^{-5}$ & $\bar{a}_{29}^{2}=8.9419 \times 10^{-7}$ & $\bar{a}_{39}^{2}=-4.4953 \times 10^{-10}$ \\
\hline $\bar{b}_{9}^{1}=-4.1118 \times 10^{-4}$ & $\bar{b}_{19}^{1}=-5.0232 \times 10^{-5}$ & $\bar{b}_{29}^{2}=-2.7632 \times 10^{-7}$ & $\bar{b}_{39}^{2}=1.7992 \times 10^{-9}$ \\
\hline $\bar{a}_{9}^{2}=-0.043314$ & $\bar{a}_{19}^{2}=-3.0592 \times 10^{-4}$ & $\bar{a}_{30}^{3}=4.9212 \times 10^{-7}$ & $\bar{a}_{40}^{3}=-6.7431 \times 10^{-10}$ \\
\hline $\bar{b}_{9}^{2}=0.13443$ & $\bar{b}_{19}^{2}=-2.8463 \times 10^{-4}$ & $\bar{b}_{30}^{3}=1.1016 \times 10^{-7}$ & $\bar{b}_{40}^{3}=7.0562 \times 10^{-10}$ \\
\hline $\bar{a}_{10}^{3}=-5.7507 \times 10^{-2}$ & $\bar{a}_{20}^{3}=-6.9235 \times 10^{-5}$ & $\bar{a}_{31}^{1}=1.5005 \times 10^{-8}$ & \\
\hline $\bar{b}_{10}^{3}=5.5903 \times 10^{-2}$ & $\bar{b}_{20}^{3}=-2.1802 \times 10^{-4}$ & $\bar{b}_{31}=-1.9320 \times 10^{-8}$ & \\
\hline
\end{tabular}

Tabela 4.3: Solução obtida via Método de Newton com chute inicial aleatório e condição de fase $\operatorname{Re}\left\{c_{1}^{1}\right\}=0 \operatorname{com} \rho=23.7715$. 


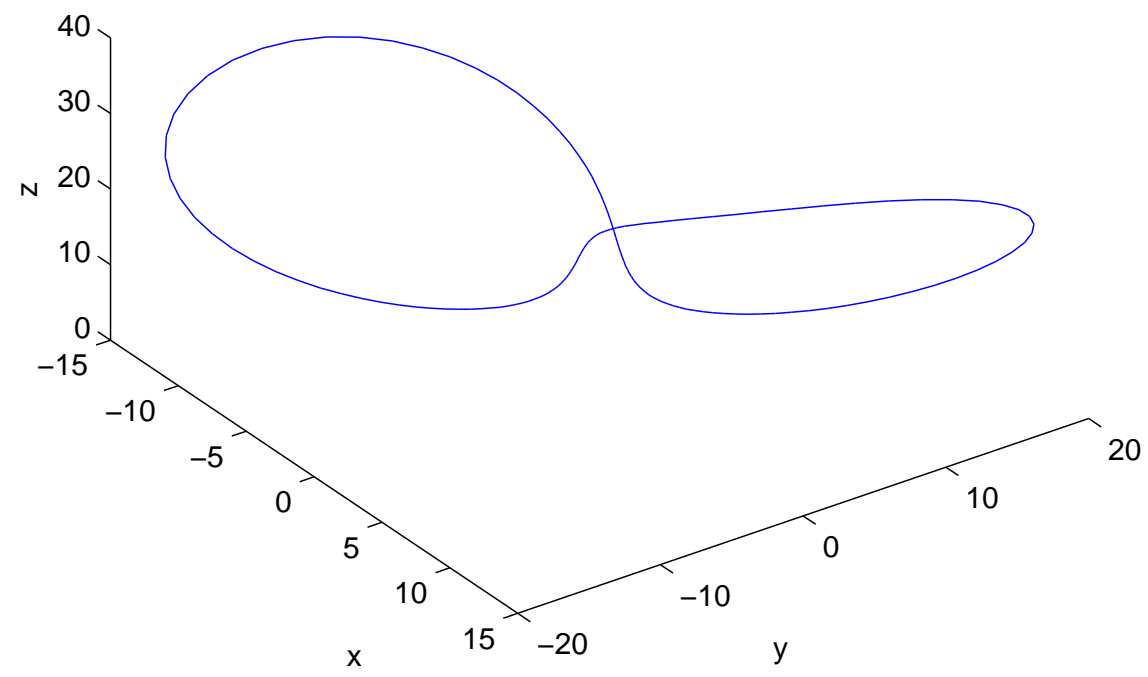

Figura 4.6: Curva periódica para o sistema de Lorenz, obtida pelo método dos polinômios radiais com chute inicial aleatório para o Método de Newton.

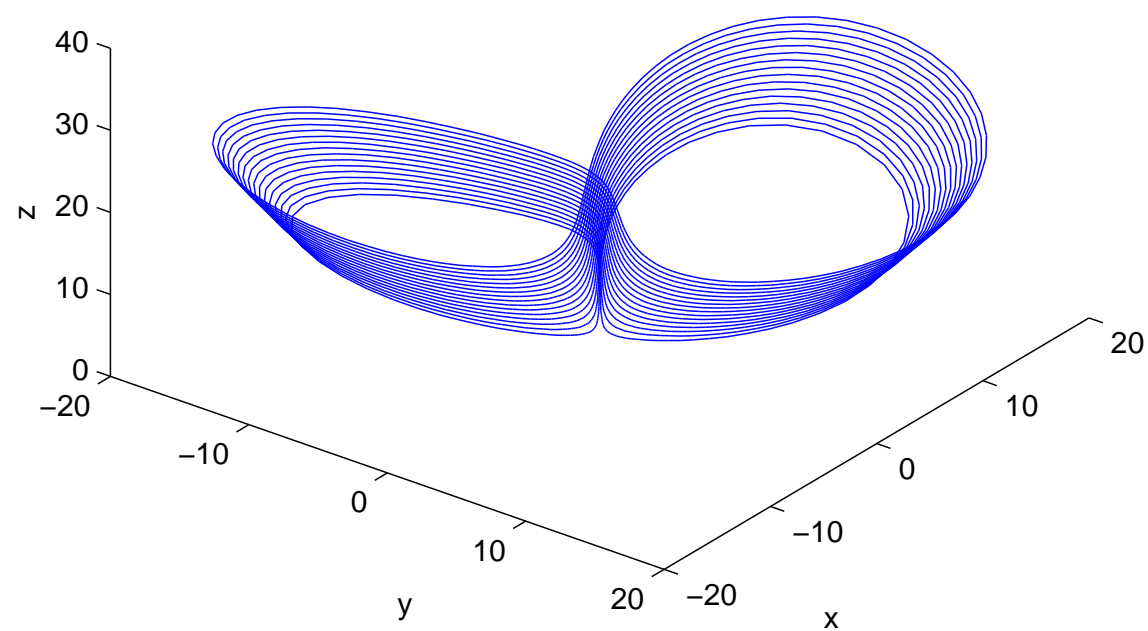

Figura 4.7: Curva de soluções obtida ao variar o parâmetro $\rho$ a partir da solução expressa na Tabela 4.3 


\section{Capítulo 5}

\section{Considerações finais}

Apesar de fornecer resultados sobre soluções de equações diferenciais ordinárias, o método proposto neste trabalho lida diretamente com o problema de determinação de pontos fixos de operadores em espaços de Banach. Podemos considerar que a essência desse método se encontra na desigualdade (4.36), uma vez que ela dá condições suficientes para que o operador de ponto fixo de interesse $T(x)=x-A F(x)$ possua pontos fixos numa bola de raio $r$ no espaço de Banach $\Omega^{s}$, no qual podemos identificar as sequências de coeficientes de Fourier das possíveis soluções periódicas que queremos obter. A introdução das cotas $Y$ e $Z(r)$ e dos polinômos radiais registra a formalização teórica adequada dessas condições.

Suponha obtida numericamente uma aproximação $\bar{c}$ para o problema $F(c)=0$. A cota Y reflete quão próximo de um ponto fixo de $T$, ou seja, de uma raiz de $F$, está a aproximação $\bar{c}$. Ou seja, está relacionada com a precisão do método numérico empregado. A cota $Z(r)$ mede quanto os pontos $T(x)$ se afastam uns dos outros conforme $x$ varia na bola $B_{\Omega^{s}}[\bar{c}, r]$. Assim, tanto $Y$ quanto $Z(r)$ avaliam se a bola $B=B_{\Omega^{s}}[\bar{c}, r]$ fica invariante por $T$, e a cota $Z(r)$ avalia se $T$ é uma contração nessa mesma bola. Dito isso, tendo em vista o Teorema do Ponto Fixo de Banach, é natural que os polinômios radiais sejam os objetos que avaliam se $T$ possui pontos fixos em $B$. Nos problemas estudados nesta dissertação, esses polinômios assumem a forma genérica $p(r)=a r^{2}+(b-1) r+c, a>$ $0, b>0, c>0$. Os coeficientes $a$ e $b$ são tais que $\sup _{u, v \in B_{\Omega^{s}}[0,1]}\left\|D T_{k}(\bar{c}+r u) r v\right\|_{\infty}=a r^{2}+b r$ e $c \geq|T(\bar{c})-\bar{c}|$. Logo, se a aproximaçao $\bar{c}$ ou a cota $Z(r)$ não forem boas, os valores de $a, b$ ou $c$ podem ser grandes. A fim de se provar a existência de soluções de $F(c)=0$, todos esses polinômios devem assumir valores negatigos para algum $r>0$. Sendo assim, o discriminante $\Delta=(b-1)^{2}-4 a c$ deve ser positivo e $b-1$ deve ser negativo, pois caso contrário $p(r)$ não tem raiz positiva e, nesse caso, o método falha. Logo, a fim de que ocorra a demonstração da existência de uma raiz de $F$ próxima à aproximação $\bar{c}$ é desejável que as cotas $Z(r)$ e $Y$ sejam pequenas.

A cota $Y$ depende do método numérico utilizado. Partindo de um chute inicial aleatório pode ocorrer que o Método de Newton para a função truncada $F^{m}$ não convirja. Pode ainda ocorrer convergência para alguma raiz aproximada de $F^{m}$ que não representa uma raiz da função completa $F$. Quanto maior a dimensão da projeção $m$ maior a chance de que, se houver convergência, esta seja para uma raiz aproximada de $F$. Sendo assim, a fim de se obter uma aproximação $\bar{c}$ razoável, podem ser necessários outros métodos numéricos diferentes do Método de Newton. O Pacote MatCont é muito útil nesse sentido. Ele também pode ser usado para fornecer chutes iniciais mais prováveis para o próprio Método de Newton. 
Se por um lado a precisão do método numérico afeta o coeficiente independente $c$ dos polinômios radiais, o operador $A$ influencia nos valores dos coeficiente $a$ e $b$, os quais, como ja citamos, precisamos que sejam pequenos. Nesse sentido, a escolha do operador

$$
A=\left(\begin{array}{l|ll}
A^{m} & & \\
\hline & {\left[\frac{\partial F_{m}}{\partial x_{m}}(\bar{c})\right]^{-1}} & \\
& & \ddots
\end{array}\right), \text { onde } A^{m} \approx\left[J F^{m}(\bar{c})\right]^{-1},
$$

é essencial para o sucesso do método dos polinômios radiais. Note que, a princípio, qualquer operador linear injetor $B$ coloca em correrspondência biunívoca as raíes de $F$ e os pontos fixos de $x \mapsto x-B F(x)$. Porém, $A$ é construído de modo a ser uma aproximação simplificada da inversa do operador $D F(\bar{c})$. Isso faz com que $D T(x)=I d-A D F(x)$ fique próximo do operador nulo numa bola pequena em torno de $\bar{c}$. Desse modo os coeficiente $a$ e $b$, que são determinados por $\sup \left|D T_{k}(\bar{c}+r u) r v\right|$, também ficam pequenos, fazendo $u, v[0,1]$

com que os determinantes dos polinômios radiais fiquem positivos e ocorram valores de $r$ tais que $p(r)<0$.

Ressalta-se aqui que a metodologia empregada na determinação de órbitas periódicas dos problemas explorados neste trabalho pode ser empregada a qualquer sistema de EDO's da forma $\dot{x}(t)=f(x(t))$, com campo vetorial $f: \mathbb{R}^{n} \rightarrow \mathbb{R}^{n}$ do tipo polinomial, ou seja, cujas funções coordenadas $f_{1}, f_{2}, \ldots, f_{n}$ são polinômios nas coordenadas $x_{1}, x_{2} \ldots x_{n}$ da variável $x$. De fato, todos esses problemas podem ser reformulados de modo a se tornarem equivalentes a um problema de determinação de raízes de uma equação da forma $F(c)=0, c \in \Omega^{s}$, para algum $s>2$ fixado, $\operatorname{com} F=\left(\eta, F_{0}, F_{1}, \ldots\right)$ e $F_{k}: \Omega^{s} \rightarrow \mathbb{R}^{2 n}$. Para isso, basta substituir na equação em estudo a função incógnita $x(t)$ pela série de Fourier correspondente. $\mathrm{O}$ aspecto das funções $F_{k}$ que essencialmente muda de acordo com a equação estudada são as ordens dos produtos de convolução que surgem de acordo com o grau dos polinômios do campo vetorial. Isso dificulta significativamente as estimativas das cotas $Y$ e $Z(r)$, tornando o método custoso, em termos práticos, para campos vetoriais com graus muito elevados, porém, ainda válido teoricamente.

Essencialmente, o método dos polinômios radiais aplica-se na determinação de pontos fixos de operadores em espaços de Banach. Sendo assim, pode-se estender sua aplicação a outros tipos de problemas que recaem, por algum processo, neste tipo de situação. Isso ocorre, por exemplo, na resolução de problemas de valores iniciais ou de fronteira. Neste caso, a base adotada no espaço de soluções é a dos polinômios de Chebychev [6]. Qualquer tipo de solução, e não só as periódicas, pode ser expressa nessa base, com a vantagem de que nela as coordenadas de uma solução qualquer decaem exponencialmente, podendo ser identificadas, no espaço de Banach $\Omega^{s}$, qualquer que seja $s>0$, já adotado nesta dissertação. As coordenadas na base de Fourier usada neste trabalho só possui esta propriedade caso as soluções procuradas sejam periódicas. Além disso, como as expressões das coordenadas das derivadas na base de Chebychev ficam muito complexas, trabalha-se com o problema integral associado à EDO, no qual as expressões das séries de Chebyschev ficam mais simples. A partir daí obtem-se um operador de ponto fixo associado ao problema, e os processos de obtenção de soluções aproximadas e de demonstração rigorosa tornam-se análogos aos descritos aqui.

Além desses problemas, dentre outras aplicações e tópicos para estudos futuros citamos a determinação da forma normal de Floquet para sistemas com campos periódicos no tempo [2], existência de soluções de equilíbrio para EDPs e unicidade global, desenvolvimentos de métodos numéricos e demonstrações computacionais rigorosas em análise de bifurcações, dentre outros problemas que surgirem durante o processo de estudo e pesquisa. 


\section{Bibliografia}

[1] Função fft. http://www.mathworks.com/help/matlab/ref/fft.html.

[2] R. Castelli and J.-P. Lessard. Rigorous numerics in floquet theory: computing stable and unstable bundles of periodic orbits. SIAM Journal on Applied Dynamical Systems, 12(1):204-245, 2013.

[3] A. Dhooge, W. Govaerts, and Y. A. Kuznetsov. Matcont: A matlab package for numerical bifurcation analysis of odes. ACM TOMS, 29:141-164, 2003.

[4] M. Gameiro, J.-P. Lessard, J. M. James, and K. Mischaikow. Computational ordinary differential equations. http://www.math.rutgers.edu/ jmireles/ODE_ course/computationalODE.pdf - Último acesso em 27/11/2014.

[5] J. K. Hale. Ordinary differential equations. Pure and Applied Mathematics, John Wiley \& Sons, New York, 1969.

[6] J.-P. Lessard and C. Reinhardt. Rigorous numerics for nonlinear differential equations using chebyshev series. SIAM Journal on Numerical Analysis, 52(1):1-22, 2014.

[7] E. L. Lima. Espaços métricos. Instituto de Matemática Pura e Aplicada, CNPq, 1977.

[8] S. Rump. INTLAB - INTerval LABoratory. In T. Csendes, editor, Developments in Reliable Computing, pages 77-104. Kluwer Academic Publishers, Dordrecht, 1999. http://www.ti3.tuhh.de/rump/.

[9] J. M. S. Tello. Lições de equações diferenciais ordinárias. Inst. de matemática pura e aplicada, 1979 .

[10] J. B. van den Berg, J. D. Mireles-James, J.-P. Lessard, and K. Mischaikow. Rigorous numerics for symmetric connecting orbits: even homoclinics of the gray-scott equation. SIAM Journal on Mathematical Analysis, 43(4):1557-1594, 2011. 